\title{
SIMULTANEOUS PROTECTION AND ACTIVATION OF AMINO ACIDS USING PROPARGYL PENTAFLUOROPHENYL CARBONATE
}

Ramapanicker Ramesh, Sakthidevi Rajasekaran, Rohit Gupta and Srinivasan Chandrasekaran*

Department of Organic Chemistry, Indian Institute of Science

Bangalore-560012, India

\section{SUPPORTING INFORMATION}

Table of Contents

\begin{tabular}{|c|c|}
\hline $\begin{array}{c}\text { Experimental Procedures and } \\
\text { Characterization Data }\end{array}$ & Page Numbers S2-S16 \\
\hline${ }^{1} \mathrm{H},{ }^{13} \mathrm{C}$ and ${ }^{19} \mathrm{~F}$ spectra of compounds & Page Numbers S17-S55 \\
\hline
\end{tabular}




\section{Experimental Procedures}

All reactions were performed in oven dry apparatus and were stirred magnetically. Melting points and optical rotation values (recorded at $25^{\circ} \mathrm{C}$ ) reported are uncorrected. Infrared spectra were recorded using an FT-IR instrument and the frequencies are reported in wave number $\left(\mathrm{cm}^{-1}\right)$ and intensities of the peaks are denoted as s (strong), w (weak), m (medium). ${ }^{1} \mathrm{H},{ }^{13} \mathrm{C}$ and ${ }^{19} \mathrm{~F}$ NMR spectra were recorded on a $300 \mathrm{MHz}, 75$ $\mathrm{MHz}$ and $282 \mathrm{MHz}$ spectrometer respectively. Chemical shifts are reported in parts per million downfield from the internal reference, tetramethylsilane for ${ }^{1} \mathrm{H}$ and ${ }^{13} \mathrm{C} \mathrm{NMR}$ and with trifluoroacetic acid as external reference for ${ }^{19} \mathrm{~F}$ NMR. Multiplicity is indicated using the following abbreviations: $\mathrm{s}$ (singlet), $\mathrm{d}$ (doublet), $\mathrm{t}$ (triplet), $\mathrm{q}$ (quartet), $\mathrm{m}$ (multiplet), sb (broad singlet) and db (broad doublet). Coupling constants are reported wherever it is necessary in Hertz $(\mathrm{Hz})$. Mass spectra were recorded on a Q-TOF electrospray instrument. Note: The carbon atoms in the pentafluorophenyl ring are not observed in the ${ }^{13} \mathrm{C}$ NMR spectra as clear single lines due to extensive coupling with ${ }^{19} \mathrm{~F}$. So the values corresponding to these peaks are not mentioned.

\section{Preparation of Propargyloxycarbonyl Chloride (PocCl$)^{5}$}

To a stirred solution of triphosgene $(2.23 \mathrm{~g}, 7.5 \mathrm{mmol})$ in dry ether $(30 \mathrm{~mL})$, activated charcoal $(0.05 \mathrm{~g})$ was added and stirred for $1 \mathrm{~h}$ at room temperature $\left(28^{\circ} \mathrm{C}\right)$. The solution was cooled to $0{ }^{\circ} \mathrm{C}$ and propargyl alcohol $(0.9 \mathrm{~mL}, 15 \mathrm{mmol})$ in dry ether $(10 \mathrm{~mL})$ was added drop wise. The resultant solution was stirred for 12 hours and filtered. The ether layer was concentrated under reduced pressure and the remaining liquid was used for reactions without any further purification.

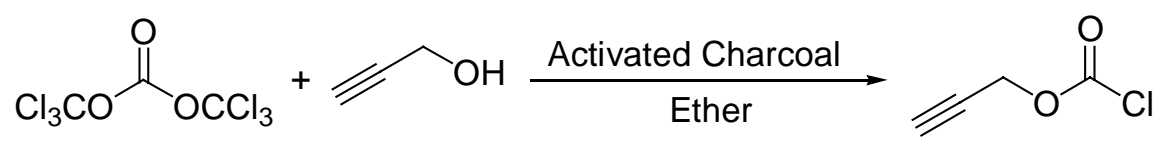

FT IR (neat):

${ }^{1} \mathrm{HNMR}\left(300 \mathrm{MHz}, \mathrm{CDCl}_{3}\right)$ :

${ }^{13} \mathrm{CNMR}\left(75 \mathrm{MHz}, \mathrm{CDCl}_{3}\right)$ :
3303, 2982, 2131, 1771

$\delta 2.6(\mathrm{t}, 1 \mathrm{H}, \mathrm{J}=2.4), 4.7(\mathrm{~d}, 2 \mathrm{H}, \mathrm{J}=2.4)$

$\delta 150.2,77.6,74.9,58.3$

\section{Preparation of Propargyl Pentafluorophenyl Carbonate (PocOPfp, 1$)^{4}$}

PocCl (10 mmol) was added to a stirred solution of pentafluorophenol $(1.84 \mathrm{~g}, 10 \mathrm{mmol})$ in dichloromethane $(30 \mathrm{~mL})$ at $-10{ }^{\circ} \mathrm{C}$. The solution was stirred for $10 \mathrm{~min}$ and triethylamine $(1.4 \mathrm{~mL}, 10 \mathrm{mmol})$ was added dropwise over a period of $15 \mathrm{~min}$. After $4 \mathrm{~h}$ 
the reaction mixture was diluted with dichloromethane $(50 \mathrm{~mL})$ and washed with $0.5 \mathrm{~N}$ $\mathrm{HCl}(20 \mathrm{~mL})$, water $(2 \times 20 \mathrm{~mL})$ and brine solution $(20 \mathrm{~mL})$. The solution containing PocOPfp was dried over anhydrous $\mathrm{Na}_{2} \mathrm{SO}_{4}$ and was purified by silica gel (100-200 mesh) column chromatography, eluting with $3 \%$ solution of ethyl acetate in hexane.

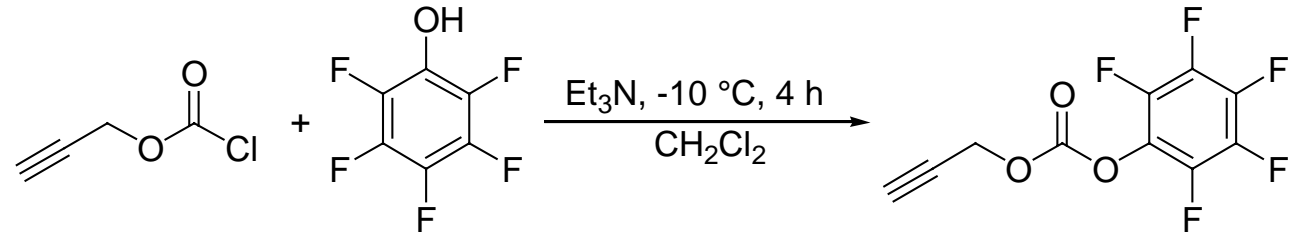

Yield:

Physical state:

Melting Point:

FTIR (KBr):

${ }^{1} \mathrm{H} \mathrm{NMR}\left(300 \mathrm{MHz}, \mathrm{CDCl}_{3}\right)$ :

${ }^{13} \mathrm{C} \mathrm{NMR}\left(75 \mathrm{MHz}, \mathrm{CDCl}_{3}\right)$ :
$98 \%$

White crystalline solid

$65^{\circ} \mathrm{C}$

$3302(\mathrm{~m}), 2134(\mathrm{w}), 1790(\mathrm{~s}), 1526(\mathrm{~s})$

$\delta 4.90(\mathrm{~d}, 2 \mathrm{H}, J=2.7), 2.65(\mathrm{t}, 1 \mathrm{H}, J=2.3)$

$\delta 150.8,142.9,139.5,138.3,138.3,136.0$,

$77.2,75.3,57.5$

\section{Preparation of Propargyloxycarbonyloxy Succinimide (PocOSu, 7)}

PocCl $(10 \mathrm{mmol})$ was added to a stirred solution of $N$-hydroxysuccinimide $(1.15 \mathrm{~g}, 10$ mmol) in dichloromethane $(30 \mathrm{~mL})$ at $-10{ }^{\circ} \mathrm{C}$. The solution was stirred for $10 \mathrm{~min}$ and triethylamine $(1.4 \mathrm{~mL}, 10 \mathrm{mmol})$ was added dropwise over a period of $15 \mathrm{~min}$. After $5 \mathrm{~h}$ the reaction mixture was diluted with dichloromethane $(50 \mathrm{~mL})$ and washed with $0.5 \mathrm{~N}$ $\mathrm{HCl}(20 \mathrm{~mL})$, water $(2 \times 20 \mathrm{~mL})$ and brine solution $(20 \mathrm{~mL})$. The solution containing PocOSu was dried over anhydrous $\mathrm{Na}_{2} \mathrm{SO}_{4}$ and was purified by silica gel (100-200 mesh) column chromatography, eluting with $20 \%$ solution of ethyl acetate in hexane.

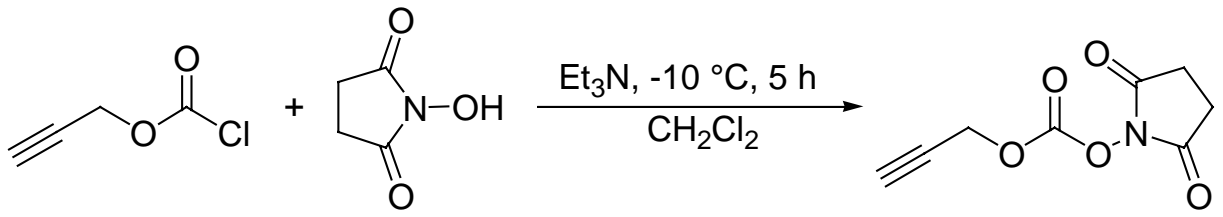

Yield:

Physical state:

Melting Point:

FTIR (KBr):
$92 \%$

White crystalline solid

$107^{\circ} \mathrm{C}$ $3262(\mathrm{~s}), 2129(\mathrm{w}), 1814(\mathrm{~s}), 1731$ (s) 
${ }^{1} \mathrm{H} \mathrm{NMR}\left(300 \mathrm{MHz}, \mathrm{CDCl}_{3}\right): \quad \delta 4.90(\mathrm{~d}, J=2.1,2 \mathrm{H}), 2.85(\mathrm{~s}, 4 \mathrm{H}), 2.66(\mathrm{t}, J=2.1$, $1 \mathrm{H})$

${ }^{13} \mathrm{C} \mathrm{NMR}\left(75 \mathrm{MHz}, \mathrm{CDCl}_{3}\right): \quad \delta 168.3,151.2,77.6,75.0,58.1,25.4$

High Resolution ESMS (m/z): Calculated for $\mathrm{C}_{8} \mathrm{H}_{7} \mathrm{NO}_{5}+\mathrm{Na}: 220.0222$

Observed: 220.0218

General Procedure for the Activation of N-Protected Amino Acids as Pentafluorophenyl Esters Using PocOPfp

A solution of the $N$-protected amino acid $(1 \mathrm{mmol})$ and PocOPfp $(0.292 \mathrm{~g}, 1.1 \mathrm{mmol})$ in DMF $(2 \mathrm{~mL})$ was cooled to $-10{ }^{\circ} \mathrm{C}$. Pyridine $(0.88 \mathrm{~mL}, 1.1 \mathrm{mmol})$ was added to this solution drop wise while stirring it magnetically. The reaction mixture was stirred for $3 \mathrm{~h}$ and was diluted with dichloromethane $(30 \mathrm{~mL})$. It is then washed with $0.5 \mathrm{~N} \mathrm{HCl}(10$ $\mathrm{mL})$, water $(2 \times 10 \mathrm{~mL})$ and brine solution $(10 \mathrm{~mL})$ and dried over anhydrous $\mathrm{Na}_{2} \mathrm{SO}_{4}$. The active esters were then purified by silica gel (100-200 mesh) column chromatography eluting with ethyl acetate-hexane mixtures of appropriate concentration (5-20\%).

\section{Boc-Pro-OPfp (4a)}

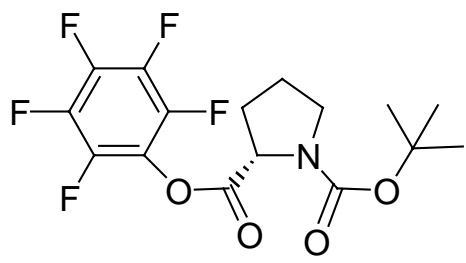

Yield:

Physical State:

Optical Rotation:

FTIR (Neat):

${ }^{1} \mathrm{H}$ NMR $\left(300 \mathrm{MHz}, \mathrm{CDCl}_{3}\right)$ :

${ }^{13} \mathrm{C} \mathrm{NMR}\left(75 \mathrm{MHz}, \mathrm{CDCl}_{3}\right)$ :

${ }^{19} \mathrm{~F}$ NMR (282 MHz, $\left.\mathrm{CDCl}_{3}\right)$ :

High Resolution ESMS (m/z):
$88 \%$ (Mixture of two rotamers)

Oily Liquid

$[\alpha]_{\mathrm{D}}-64.00(\mathrm{c}=1.0$, ethanol $)$

$1797(\mathrm{~s}), 1705$ (s)

$\delta$ 4.66-4.57 (m, 1H), 3.67-3.41 (m, 2H), 2.51-2.35 (m, 1H), 2.35-2.18 (m, 1H)-2.11-1.91 (m, 2H), 1.47 $(\mathrm{d}, 9 \mathrm{H})$ $\delta 169.2,169.0,80.8,80.4,77.4,77.0,76.5,58.5$, 58.5, 46.5, 46.3, 31.2, 30.0, 28.2, 28.0, 24.4, 23.4 $\delta-40.73$ (d, 2F), -45.94 (t, 2F), -50.39 (t, 1F)

Calculated for $\mathrm{C}_{16} \mathrm{H}_{16} \mathrm{~F}_{5} \mathrm{NO}_{4}+\mathrm{Na}: 404.0897$ Observed: 404.0902 


\section{Boc-Leu-OPfp (4b)}

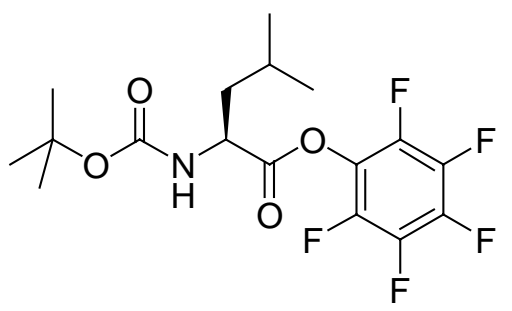

Yield:

$89 \%$

Physical State:

Oily Liquid

Optical Rotation:

$[\alpha]_{\mathrm{D}}-13.00(\mathrm{c}=1.0$, ethanol)

FTIR (Neat):

$3289(\mathrm{~m}), 1777$ (s), 1710 (s)

${ }^{1} \mathrm{H}$ NMR (300 MHz, $\left.\mathrm{CDCl}_{3}\right)$ :

$\delta 4.90(\mathrm{~d}, J=2.4,1 \mathrm{H}), 4.66-4.59$ (m, 1H), 1.87-1.62

(m, 3H), $1.46(\mathrm{~s}, 9 \mathrm{H}), 1.01(\mathrm{~d}, J=6.0,6 \mathrm{H})$

${ }^{13} \mathrm{C} \mathrm{NMR}\left(75 \mathrm{MHz}, \mathrm{CDCl}_{3}\right): \quad \delta 169.6,155.2,80.5,77.4,77.0,76.5,52.1,41.1$, $28.2,24.8,22.7,21.7$

${ }^{19} \mathrm{~F} \mathrm{NMR}\left(282 \mathrm{MHz}, \mathrm{CDCl}_{3}\right): \quad \delta-40.60(\mathrm{~d}, 2 \mathrm{~F}),-46.02(\mathrm{t}, 2 \mathrm{~F}),-50.52(\mathrm{t}, 1 \mathrm{~F})$

High Resolution ESMS (m/z): $\quad$ Calculated for $\mathrm{C}_{17} \mathrm{H}_{20} \mathrm{~F}_{5} \mathrm{NO}_{4}+\mathrm{Na}: 420.1210$

Observed: 420.1211

\section{Cbz-Phe-OPfp (4c)}<smiles>O=C(N[C@@H](Cc1ccccc1)C(=O)Oc1c(F)c(F)c(F)c(F)c1F)OCc1ccccc1</smiles>

Yield:

$85 \%$

Physical State:

White crystalline solid

Melting Point:

$96{ }^{\circ} \mathrm{C}$

Optical Rotation:

$[\alpha]_{\mathrm{D}}+54.00(\mathrm{c}=1.0$, ethanol $)$

FTIR (KBr):

3322 (m), 1791 (s), 1714 (s) 


\author{
${ }^{1} \mathrm{H}$ NMR $\left(300 \mathrm{MHz}, \mathrm{CDCl}_{3}\right): \quad \delta 7.37-7.19(\mathrm{~m}, 10 \mathrm{H}), 5.47(\mathrm{~d}, J=7.8,1 \mathrm{H}), 5.10(\mathrm{~s}$, \\ $2 \mathrm{H}), 4.5 .06-4.97(\mathrm{~m}, 1 \mathrm{H}), 3.35-3.18(\mathrm{~m}, 2 \mathrm{H})$ \\ ${ }^{13} \mathrm{C}$ NMR $\left(75 \mathrm{MHz}, \mathrm{CDCl}_{3}\right): \quad \delta 168.0,155.5,135.8,134.5,129.2,128.9,128.5$, \\ $128.2,128.1,127.5,67.3,54.6,37.7$ \\ ${ }^{19} \mathrm{~F}$ NMR $\left(282 \mathrm{MHz}, \mathrm{CDCl}_{3}\right): \quad \delta-40.03(\mathrm{~d}, 2 \mathrm{~F}),-45.44(\mathrm{t}, 3 \mathrm{~F}),-50.12(\mathrm{t}, 1 \mathrm{~F})$ \\ High Resolution ESMS: $\quad$ Calculated for $\mathrm{C}_{23} \mathrm{H}_{16} \mathrm{~F}_{5} \mathrm{NO}_{4}+\mathrm{Na}: 488.0897$ \\ Observed: 488.0900
}

\title{
General Procedure for the Simultaneous Protection and Activation of Amino Acids Using PocOPfp
}

A solution of the amino acid (1 mmol) and PocOPfp (0.559 g, $2.1 \mathrm{mmol})$ in DMF (2 mL) was cooled to $-10{ }^{\circ} \mathrm{C}$ and pyridine $(0.177 \mathrm{~mL}, 2.2 \mathrm{mmol})$ was added to it dropwise. The reaction mixture was allowed to attain room temperature slowly and was stirred for 3-5 hours. The reaction mixture was then diluted with dichloromethane $(30 \mathrm{~mL})$ and washed with $0.5 \mathrm{~N} \mathrm{HCl}(10 \mathrm{~mL})$, water $(2 \times 10 \mathrm{~mL})$ and brine $(10 \mathrm{~mL})$. The resulting solution was dried over $\mathrm{Na}_{2} \mathrm{SO}_{4}$ and concentrated under vacuum and the products were purified by silica gel (100-200 mesh) column chromatography eluting with ethyl acetate hexane mixtures of appropriate concentration (5-20\%).

\section{Pentafluorophenyl $m$-( $N$-Poc)aminobenzoate (6a)}<smiles>C#CCOC(=O)Nc1cccc(C(=O)Oc2c(F)c(F)c(F)c(F)c2F)c1</smiles>

Yield:

Physical State:

Melting Point:

FTIR (KBr):

${ }^{1} \mathrm{H}$ NMR $\left(300 \mathrm{MHz}, \mathrm{CDCl}_{3}\right)$ :
$92 \%$

White crystalline solid

$99{ }^{\circ} \mathrm{C}$

3309 (m), 2130 (w), 1758 (s), 1716 (s)

$\delta 8.20(\mathrm{~m}, 1 \mathrm{H}), 7.92-7.89(\mathrm{~m}, 1 \mathrm{H}), 7.81-7.78(\mathrm{~m}$,

$1 \mathrm{H}), 7.49(\mathrm{t}, J=8.1,1 \mathrm{H}), 7.12(\mathrm{~s}, 1 \mathrm{H}), 4.80(\mathrm{~d}$, $J=2.1,2 \mathrm{H}), 2.53(\mathrm{t}, J=2.1,1 \mathrm{H})$ 
${ }^{13} \mathrm{C} \mathrm{NMR}\left(75 \mathrm{MHz}, \mathrm{CDCl}_{3}\right): \quad \delta 162.2,152.4,138.2,129.7,127.7,125.8,124.7$, $120.5,77.4,75.2,55.0$

${ }^{19} \mathrm{~F} \mathrm{NMR}\left(282 \mathrm{MHz}, \mathrm{CDCl}_{3}\right): \quad \delta-40.42(\mathrm{~d}, 2 \mathrm{~F}),-45.52(\mathrm{t}, 1 \mathrm{~F}),-50.28(\mathrm{t}, 2 \mathrm{~F})$

High Resolution ESMS (m/z): $\quad$ Calculated for $\mathrm{C}_{17} \mathrm{H}_{8} \mathrm{~F}_{5} \mathrm{NO}_{4}+\mathrm{H}: 386.0451$

Observed: 386.0449

Pentafluorophenyl $o$-( $N$-Poc)aminobenzoate (6b)

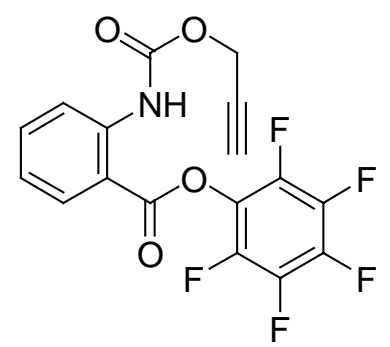

Yield:

Physical State:

Melting Point:

FTIR (KBr):

${ }^{1} \mathrm{H}$ NMR (300 MHz, $\left.\mathrm{CDCl}_{3}\right)$ :

${ }^{13} \mathrm{C}$ NMR (75 MHz, $\left.\mathrm{CDCl}_{3}\right)$ :

${ }^{19} \mathrm{~F}$ NMR (282 MHz, $\left.\mathrm{CDCl}_{3}\right)$ :

High Resolution ESMS (m/z):

\section{$90 \%$}

White solid

$125^{\circ} \mathrm{C}$

$3280(\mathrm{~m}), 2138$ (w), 1783 (s), 1722 (s)

$\delta 10.02(\mathrm{~s}, 1 \mathrm{H}), 8.55-8.52(\mathrm{~m}, 1 \mathrm{H}), 8.26(\mathrm{~m}, 1 \mathrm{H})$, 7.72-7.66 (m, $1 \mathrm{H}), 7.26-7.15(\mathrm{~m}, 1 \mathrm{H}), 4.78(\mathrm{~d}$, $J=2.4,2 \mathrm{H}), 2.50(\mathrm{t}, J=2.4,1 \mathrm{H})$

$\delta 164.0,152.3,142.5,136.6,131.7,122.3,119.2$, $111.6,77.4,75.1,52.9$

$\delta-40.63(\mathrm{~d}, 2 \mathrm{~F}),-45.49(\mathrm{t}, 1 \mathrm{~F}),-50.31(\mathrm{t}, 2 \mathrm{~F})$

Calculated for $\mathrm{C}_{17} \mathrm{H}_{8} \mathrm{~F}_{5} \mathrm{NO}_{4}+\mathrm{Na}$ : 408.0271

Observed: 408.0275

\section{Poc-Leu-OPfp (6c)}<smiles>C#CCOC(=O)NC(CC(C)C)C(=O)Oc1c(F)c(F)c(F)c(F)c1F</smiles>

Yield:

$82 \%$ 
Physical State:

Optical Rotation:

FTIR (Neat):

${ }^{1} \mathrm{H}$ NMR (300 MHz, $\left.\mathrm{CDCl}_{3}\right)$ :

${ }^{13} \mathrm{C} \mathrm{NMR}\left(75 \mathrm{MHz}, \mathrm{CDCl}_{3}\right)$ :

${ }^{19} \mathrm{~F}$ NMR (282 MHz, $\left.\mathrm{CDCl}_{3}\right)$ :

High Resolution ESMS (m/z):

\section{Poc-Ala-OPfp (6d)}

Yield:

Physical State:

Optical Rotation:

FTIR (Neat):

${ }^{1} \mathrm{H}$ NMR (300 MHz, $\left.\mathrm{CDCl}_{3}\right)$ :

${ }^{13} \mathrm{C}$ NMR (75 MHz, $\left.\mathrm{CDCl}_{3}\right)$ :

${ }^{19} \mathrm{~F}$ NMR (282 MHz, $\left.\mathrm{CDCl}_{3}\right)$ :

High Resolution ESMS (m/z):
Gummy solid

$[\alpha]_{\mathrm{D}}-166.00(\mathrm{c}=1.0$, ethanol $)$

$3318(\mathrm{~m}), 2126(\mathrm{w}), 1793(\mathrm{~s}), 1718(\mathrm{~s})$

$\delta 5.36(\mathrm{~d}, J=8.1,1 \mathrm{H}), 4.79-4.66(\mathrm{~m}, 3 \mathrm{H}), 2.50(\mathrm{t}$, $J=2.4,1 \mathrm{H}), 1.86-1.69$ (m, 3H), 1.01 (d, $J=5.7,6 \mathrm{H})$ $\delta 169.2,155.0,77.4,75.0,53.1,52.4,41.1,24.7$, 22.6, 21.5

$\delta-40.70(\mathrm{~d}, 2 \mathrm{~F}),-45.65(\mathrm{t}, 1 \mathrm{~F}),-50.36(\mathrm{t}, 2 \mathrm{~F})$

Calculated for $\mathrm{C}_{16} \mathrm{H}_{14} \mathrm{~F}_{5} \mathrm{NO}_{4}+\mathrm{Na}: 402.0741$

Observed: 402.0749<smiles>C#CCOC(=O)N[C@@H](C)C(=O)Oc1c(F)c(F)c(F)c(F)c1F</smiles>

$68 \%$

Gummy solid

$[\alpha]_{\mathrm{D}}+11.00(\mathrm{c}=1.0$, ethanol $)$

3297 (m), 2129 (w), 1781 (s), 1714 (s)

$\delta 5.41(\mathrm{~d}, J=7.2,1 \mathrm{H}), 4.78-4.66(\mathrm{~m}, 3 \mathrm{H}), 2.50(\mathrm{t}$, $J=2.4,1 \mathrm{H}), 1.63(\mathrm{~d}, J=7.2,3 \mathrm{H})$

$\delta 169.2,154.6,77.4,75.0,53.0,49.5,18.2$

$\delta-40.95(\mathrm{~d}, 2 \mathrm{~F}),-45.49(\mathrm{t}, 1 \mathrm{~F}),-50.20(\mathrm{t}, 2 \mathrm{~F})$

Calculated $\mathrm{C}_{13} \mathrm{H}_{8} \mathrm{~F}_{5} \mathrm{NO}_{4}+\mathrm{Na}: 360.0271$

Observed: 360.0274

\section{Poc-Val-OPfp (6e)}<smiles>C#CCOC(=O)NC(C(=O)Oc1c(F)c(F)c(F)c(F)c1F)C(C)C</smiles>

Yield: 
Physical State:

Optical Rotation:

FTIR (Neat):

${ }^{1} \mathrm{H}$ NMR (300 MHz, $\left.\mathrm{CDCl}_{3}\right)$ :

${ }^{13} \mathrm{C} \mathrm{NMR}\left(75 \mathrm{MHz}, \mathrm{CDCl}_{3}\right)$ :

${ }^{19}$ F NMR (282 MHz, $\left.\mathrm{CDCl}_{3}\right)$ :

High Resolution ESMS (m/z):
Gummy solid

$[\alpha]_{\mathrm{D}}+13.00(\mathrm{c}=1.0$, ethanol $)$

3313 (m), 2129 (w), 1787 (s), 1720 (s)

$\delta 5.43(\mathrm{~d}, J=9.3,1 \mathrm{H}), 4.80-4.64(\mathrm{~m}, 3 \mathrm{H}), 2.51(\mathrm{t}$, $J=2.4,1 \mathrm{H}), 2.45-2.35(\mathrm{~m}, 1 \mathrm{H}), 1.10$ (d, $J=6.6,3 \mathrm{H})$, $1.03(\mathrm{~d}, J=7.2,3 \mathrm{H})$

$\delta 168.1,155.2,77.4,75.0,59.0,53.1,31.1,18.8$, 17.1

$\delta-40.42(\mathrm{~d}, 2 \mathrm{~F}),-45.52(\mathrm{t}, 1 \mathrm{~F}),-50.28(\mathrm{t}, 2 \mathrm{~F})$

Calculated for $\mathrm{C}_{15} \mathrm{H}_{12} \mathrm{~F}_{5} \mathrm{NO}_{4}+\mathrm{Na}: 388.0584$

Observed: 388.0591

\section{Poc-Ile-OPfp (6f)}<smiles>C#CCOC(=O)NC(C(=O)Oc1c(F)c(F)c(F)c(F)c1F)C(C)CC</smiles>

Yield:

Physical State:

Optical Rotation:

FTIR (Neat):

${ }^{1} \mathrm{H}$ NMR (300 MHz, $\left.\mathrm{CDCl}_{3}\right)$ :

${ }^{13} \mathrm{C} \mathrm{NMR}\left(75 \mathrm{MHz}, \mathrm{CDCl}_{3}\right)$ :

${ }^{19}$ F NMR (282 MHz, $\left.\mathrm{CDCl}_{3}\right)$ :

High Resolution ESMS (m/z):
$84 \%$

Gummy solid

$[\alpha]_{\mathrm{D}}+26.00(\mathrm{c}=1.0$, ethanol $)$

$3313(\mathrm{~m}), 2130(\mathrm{w}), 1789(\mathrm{~s}), 1720$ (s)

$\delta 5.39(\mathrm{~d}, J=8.7,1 \mathrm{H}), 4.79-4.67(\mathrm{~m}, 3 \mathrm{H}), 2.50(\mathrm{t}$, $J=2.4,1 \mathrm{H}), 2.16-2.01(\mathrm{~m}, 1 \mathrm{H}), 1.59-1.48(\mathrm{~m}, 1 \mathrm{H})$, 1.36-1.21 (m, 1H), $1.07(\mathrm{~d}, J=6.9,3 \mathrm{H}), 0.99$ (t, $J=7.2,3 \mathrm{H})$

$\delta 168.1,155.1,77.4,75.0,58.4,53.1,37.8,24.7$, $15.3,11.4$

$\delta-40.29(\mathrm{~d}, 2 \mathrm{~F}),-45.46(\mathrm{t}, 1 \mathrm{~F}),-50.20(\mathrm{t}, 2 \mathrm{~F})$

Calculated for $\mathrm{C}_{16} \mathrm{H}_{14} \mathrm{~F}_{5} \mathrm{NO}_{4}+\mathrm{K}: 418.0480$

Observed: 418.0479 


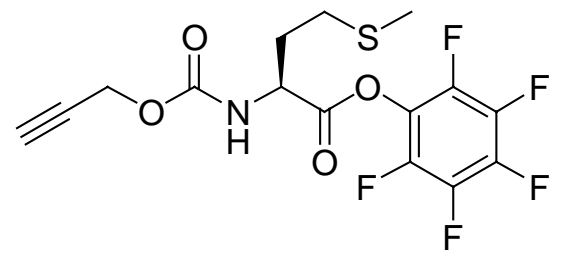

Yield:

Physical State:

Optical Rotation:

FTIR (Neat):

${ }^{1} \mathrm{H}$ NMR (300 MHz, $\left.\mathrm{CDCl}_{3}\right)$ :

${ }^{13} \mathrm{C}$ NMR $\left(75 \mathrm{MHz}, \mathrm{CDCl}_{3}\right)$ :

${ }^{19} \mathrm{~F}$ NMR (282 MHz, $\left.\mathrm{CDCl}_{3}\right)$ :

High Resolution ESMS (m/z):

$72 \%$

Gummy solid

$[\alpha]_{D}-17.00(\mathrm{c}=1.0$, ethanol)

$3286(\mathrm{~m}), 2125$ (w), 1790 (s), 1718 (s)

$\delta 5.69(\mathrm{~d}, J=7.8,1 \mathrm{H}), 4.93-4.99(\mathrm{~m}, 1 \mathrm{H}), 4.80-4.67$ (m, 2H), 2.73-2.61 (m, 2H), $2.51(\mathrm{t}, J=2.4,1 \mathrm{H})$, 2.40-2.29 (m, 1H), 2.24-2.14 (m, 4H)

$\delta 168.3,154.9,77.4,75.1,53.2,53.0,31.3,29.6$, 15.3

$\delta-40.69$ (d, 2F), $-45.23(\mathrm{t}, 1 \mathrm{~F}),-50.04(\mathrm{t}, 2 \mathrm{~F})$

Calculated for $\mathrm{C}_{15} \mathrm{H}_{12} \mathrm{~F}_{5} \mathrm{NO}_{4}+\mathrm{Na}: 420.0305$

Observed: 420.0300

\section{Poc-Phe-OPfp (6h)}

Yield:

Physical State:

Melting Point:

Optical Rotation:

FTIR (KBr):

${ }^{1} \mathrm{H}$ NMR (300 MHz, $\left.\mathrm{CDCl}_{3}\right)$ :<smiles>C#CCOC(=O)NC(Cc1ccccc1)C(=O)Oc1c(F)c(F)c(F)c(F)c1F</smiles>

$73 \%$

White Solid

$82{ }^{\circ} \mathrm{C}$

$[\alpha]_{\mathrm{D}}+12.00(\mathrm{c}=1.0$, ethanol $)$

3305 (m), 2129 (w), 1793 (s), 1718 (s)

$\delta$ 7.38-7.21 (m, 5H), 5.29 (d, $J=8.1,1 \mathrm{H}), 5.03-4.96$

(m, 1H), 4.74-4.62 (m, 2H), 3.37-3.21 (m, 2H), 2.48

(t, $J=2.4,1 \mathrm{H})$ 
${ }^{13} \mathrm{C} \mathrm{NMR}\left(75 \mathrm{MHz}, \mathrm{CDCl}_{3}\right): \quad \delta 167.8,154.6,134.3,129.2,28.9,127.6,77.4,75.0$, $54.6,53.1,37.7$

${ }^{19} \mathrm{~F} \mathrm{NMR}\left(282 \mathrm{MHz}, \mathrm{CDCl}_{3}\right): \quad \delta-40.05(\mathrm{~d}, 2 \mathrm{~F}),-45.31(\mathrm{t}, 1 \mathrm{~F}),-50.04(\mathrm{t}, 2 \mathrm{~F})$

High Resolution ESMS (m/z): Calculated for $\mathrm{C}_{19} \mathrm{H}_{12} \mathrm{~F}_{5} \mathrm{NO}_{4}+\mathrm{H}$ : 414.0764

Observed: 414.0766

\section{Poc-Pro-OPfp (6i)}

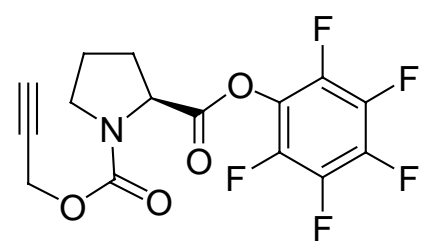

Yield:

$77 \%$ (Mixture of two rotamers)

Physical Appearance: $\quad$ Gummy solid

Optical Rotation: $\quad[\alpha]_{\mathrm{D}}-292(\mathrm{c}=1.0$, ethanol $)$

FTIR (Neat): $\quad 3297(\mathrm{~m}), 2130(\mathrm{w}), 1783(\mathrm{~s}), 1687$ (s)

${ }^{1} \mathrm{H}$ NMR $\left(300 \mathrm{MHz}, \mathrm{CDCl}_{3}\right): \quad \delta 4.84-4.61(\mathrm{~m}, 3 \mathrm{H}), 3.74-3.45(\mathrm{~m}, 2 \mathrm{H}), 2.55-1.93$

$(\mathrm{m}, 5 \mathrm{H})$

${ }^{13} \mathrm{C}$ NMR $\left(75 \mathrm{MHz}, \mathrm{CDCl}_{3}\right): \quad \delta 177.7,176.4,154.8,153.8,77.9,74.8,74.7,59.2$,

58.6, 53.3, 53.1, 46.9, 46.6, 30.8, 29.4, 24.2, 23.3

${ }^{19}$ F NMR $\left(282 \mathrm{MHz}, \mathrm{CDCl}_{3}\right): \quad \delta-40.939(\mathrm{~d}),-41.39(\mathrm{~d}),-45.78(\mathrm{t}),-46.07(\mathrm{t}),-$ $50.41(\mathrm{t}),-50.62(\mathrm{t})$

High Resolution ESMS (m/z): Calculated for $\mathrm{C}_{15} \mathrm{H}_{10} \mathrm{~F}_{5} \mathrm{NO}_{4}+\mathrm{Na}: 386.0428$

Observed: 386.0438

\section{Poc-Aib-OPfp (6j)}<smiles>C#CCOC(=O)NC(C)(C)C(=O)Oc1c(F)c(F)c(F)c(F)c1F</smiles>

Yield:

$76 \%$

Physical Appearance: $\quad$ Gummy Solid

FTIR (Neat): $\quad 3313(\mathrm{~m}), 2130(\mathrm{w}), 1793(\mathrm{~s}), 1718(\mathrm{~s})$ 
${ }^{1} \mathrm{H}$ NMR $\left(300 \mathrm{MHz}, \mathrm{CDCl}_{3}\right): \quad \delta 5.48(\mathrm{~s}, 1 \mathrm{H}), 4.72(\mathrm{~d}, J=2.4,2 \mathrm{H}), 2.48(\mathrm{t}, J=2.4$, $1 \mathrm{H}), 1.71(\mathrm{~s}, 6 \mathrm{H})$

${ }^{13} \mathrm{C} \mathrm{NMR}\left(75 \mathrm{MHz}, \mathrm{CDCl}_{3}\right): \quad \delta 170.3,154.0,77.2,74.9,56.8,52.8,25.1$

${ }^{19} \mathrm{~F} \mathrm{NMR}\left(282 \mathrm{MHz}, \mathrm{CDCl}_{3}\right): \quad \delta-40.98(\mathrm{~d}, 2 \mathrm{~F}),-46.12(\mathrm{t}, 1 \mathrm{~F}),-50.70(\mathrm{t}, 2 \mathrm{~F})$

High Resolution ESMS (m/z): Calculated for $\mathrm{C}_{14} \mathrm{H}_{10} \mathrm{~F}_{5} \mathrm{NO}_{4}+\mathrm{H}: 352.0608$

Observed: 352.0599

\section{Poc-(ß)Ala-OPfp (6k)}<smiles>C#CCOC(=O)NCCC(=O)Oc1c(F)c(F)c(F)c(F)c1F</smiles>

Yield:

$60 \%$

Physical Appearance:

Gummy solid

FTIR (Neat):

$3311(\mathrm{~m}), 2130(\mathrm{w}), 1787(\mathrm{~s}), 1714(\mathrm{~s})$

${ }^{1} \mathrm{H}$ NMR (300 MHz, $\mathrm{CDCl}_{3}$ ):

$\delta 5.36(\mathrm{sb}, 1 \mathrm{H}), 4.70(\mathrm{~d}, J=2.4,2 \mathrm{H}), 3.61$ (q, $J=6.3$, $2 \mathrm{H}), 2.96(\mathrm{t}, \mathrm{J}=6.3,2 \mathrm{H}), 2.49(\mathrm{t}, \mathrm{J}=2.4,1 \mathrm{H})$

${ }^{13} \mathrm{C}$ NMR $\left(75 \mathrm{MHz}, \mathrm{CDCl}_{3}\right)$ :

$\delta 168.2,155.4,77.9,74.7,52.6,36.3,33.6$

${ }^{19} \mathrm{~F}$ NMR (282 MHz, $\left.\mathrm{CDCl}_{3}\right)$ :

$\delta-40.98(\mathrm{~d}, 2 \mathrm{~F}),-45.91(\mathrm{t}, 1 \mathrm{~F}),-50.43(\mathrm{t}, 2 \mathrm{~F})$

High Resolution ESMS (m/z): Calculated for $\mathrm{C}_{13} \mathrm{H}_{8} \mathrm{~F}_{5} \mathrm{NO}_{4}+\mathrm{Na}$ : 360.0271

Observed: 360.0283

\section{Poc-Gaba-OPfp (61)}

Yield:

Physical State:

Melting Point:<smiles>C#CCOC(=O)NCCCC(=O)Oc1c(F)c(F)c(F)c(F)c1F</smiles>

$68 \%$

White solid

$71{ }^{\circ} \mathrm{C}$ 
FTIR (KBr):

${ }^{1} \mathrm{H} \mathrm{NMR}\left(300 \mathrm{MHz}, \mathrm{CDCl}_{3}\right)$ :

${ }^{13} \mathrm{C}$ NMR (75 MHz, $\left.\mathrm{CDCl}_{3}\right)$ :

${ }^{19} \mathrm{~F}$ NMR (282 $\left.\mathrm{MHz}, \mathrm{CDCl}_{3}\right)$ :

High Resolution ESMS (m/z):
3309 (m), 2129 (w), 1789 (s), 1710 (s)

$\delta 5.08(\mathrm{~s}, 1 \mathrm{H}), 4.69$ (d, $J=2.4,2 \mathrm{H}), 3.33$ (q, $J=6.6$, $2 \mathrm{H}), 2.75(\mathrm{t}, J=7.5,2 \mathrm{H}), 2.48(\mathrm{t}, J=2.4,1 \mathrm{H}), 2.04-$ $1.95(\mathrm{~m}, 2 \mathrm{H})$ $\delta 169.0,155.6,78.0,74.6,52.5,40.0,30.4,24.9$ $\delta-41.19(\mathrm{~d}, 2 \mathrm{~F}),-46.31(\mathrm{t}, 2 \mathrm{~F}),-50.68(\mathrm{t}, 1 \mathrm{~F})$ Calculated for $\mathrm{C}_{14} \mathrm{H}_{10} \mathrm{~F}_{5} \mathrm{NO}_{4}+\mathrm{H}: 352.0608$ Observed: 352.0608

\section{Poc-Phg-OPfp (6m)}

Yield:

Physical State:

Optical Rotation:

FTIR (KBr):

${ }^{1} \mathrm{H}$ NMR (300 MHz, $\left.\mathrm{CDCl}_{3}\right)$ :

${ }^{13} \mathrm{C} \mathrm{NMR}\left(75 \mathrm{MHz}, \mathrm{CDCl}_{3}\right)$ :

${ }^{19}$ F NMR (282 MHz, $\left.\mathrm{CDCl}_{3}\right)$ :

High Resolution ESMS (m/z):<smiles>C#CCOC(=O)N[C@H](C(=O)Oc1c(F)c(F)c(F)c(F)c1F)c1ccccc1</smiles>

$75 \%$

Gummy solid

$[\alpha]_{\mathrm{D}}+13.00(\mathrm{c}=1.0$, ethanol $)$

$3321(\mathrm{~m}), 2124(\mathrm{w}), 1795$ (s), 1714 (s)

$\delta$ 7.46-7.42 (m, 5H), $5.84(\mathrm{db}, J=6.9,1 \mathrm{H}), 5.72(\mathrm{~d}$, $J=6.9,1 \mathrm{H}), 4.72(\mathrm{~d}, J=2.1,2 \mathrm{H}), 2.48(\mathrm{t}, J=2.1,1 \mathrm{H})$ $\delta 167.2,154.4,134.2,129.4,129.3,127.4,77.5$, 75.1, 57.9, 53.2

$\delta-40.08(\mathrm{~d}, 2 \mathrm{~F}),-45.33(\mathrm{t}, 2 \mathrm{~F}),-50.10(\mathrm{t}, 1 \mathrm{~F})$

Calculated for $\mathrm{C}_{18} \mathrm{H}_{10} \mathrm{~F}_{5} \mathrm{NO}_{4}+\mathrm{H}: 422.0428$

Observed: 422.0435 


\section{Cbz-Lys(Poc)-OPfp (6n)}

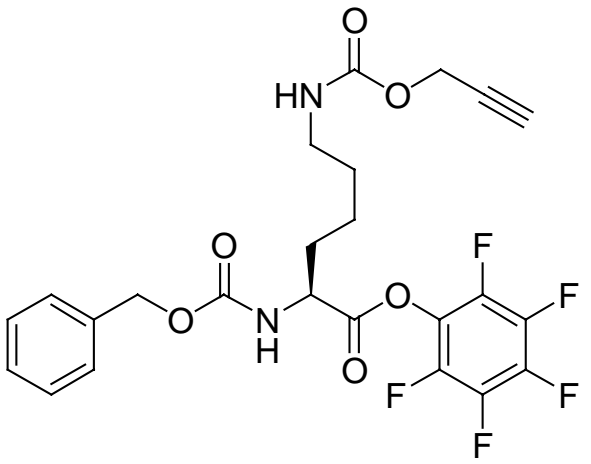

Yield:

Physical Appearance:

Optical Rotation:

FTIR (Neat):

${ }^{1} \mathrm{H}$ NMR (300 MHz, $\mathrm{CDCl}_{3}$ ):

${ }^{13} \mathrm{C}$ NMR (75 MHz, $\left.\mathrm{CDCl}_{3}\right)$ :

$\left.{ }^{19} \mathrm{~F} \mathrm{NMR} \mathrm{(282} \mathrm{MHz,} \mathrm{CDCl}_{3}\right)$ :

High Resolution ESMS (m/z):
$76 \%$

Gummy solid

$[\alpha]_{\mathrm{D}}-110.00(\mathrm{c}=1.0$, ethanol)

3299 (m), 2127 (w), 1791 (s), 1706 (s)

$\delta$ 7.35-7.26 (m, 5H), $5.57(\mathrm{~d}, J=6.6,1 \mathrm{H}), 5.14(\mathrm{~s}$, $2 \mathrm{H}), 4.94(\mathrm{sb}, 1 \mathrm{H}), 4.73-4.63(\mathrm{~m}, 3 \mathrm{H}), 3.25-3.15$ (m, 2H), $2.44(\mathrm{~s}, 1 \mathrm{H}), 2.06-1.84(\mathrm{~m}, 2 \mathrm{H}), 1.61-1.46$ $(\mathrm{m}, 4 \mathrm{H})$

$\delta 168.7,155.9,155.7,135.8,128.5,128.2,128.1$, 78.1, 74.5, 67.3, 53.5, 52.4, 40.2, 31.4, 29.2, 29.1

$\delta-40.68(\mathrm{~d}, 2 \mathrm{~F}),-45.52(\mathrm{t}, 1 \mathrm{~F}),-50.18(\mathrm{t}, 2 \mathrm{~F})$

Calculated for $\mathrm{C}_{24} \mathrm{H}_{21} \mathrm{~F}_{5} \mathrm{~N}_{2} \mathrm{O}_{6}+\mathrm{Na}: 551.1218$

Observed: 551.1223

\section{Poc-Lys(Poc)-OPfp (6o)}<smiles>C#CCOC(=O)NCCCCC(NC(=O)OCC#C)C(=O)Oc1c(F)c(F)c(F)c(F)c1F</smiles>

Yield:

Physical Appearance:

Optical Rotation:
$57 \%$

Gummy solid

$[\alpha]_{D}-10.00(\mathrm{c}=1.0$, ethanol $)$ 
FTIR (Neat):

${ }^{1} \mathrm{H}$ NMR (300 MHz, $\left.\mathrm{CDCl}_{3}\right)$ :

${ }^{13} \mathrm{C} \mathrm{NMR}\left(75 \mathrm{MHz}, \mathrm{CDCl}_{3}\right)$ :

${ }^{19} \mathrm{~F}$ NMR (282 MHz, $\left.\mathrm{CDCl}_{3}\right)$ :

High Resolution ESMS (m/z):

\section{Poc-Glu(Pfp)-OPfp (6p)}

Yield:

Physical Appearance:

Optical Rotation:

FTIR (Neat):

${ }^{1} \mathrm{H} \mathrm{NMR}\left(300 \mathrm{MHz}, \mathrm{CDCl}_{3}\right)$

${ }^{13} \mathrm{C}$ NMR (75 MHz, $\left.\mathrm{CDCl}_{3}\right)$ :

${ }^{19} \mathrm{~F}$ NMR (282 MHz, $\left.\mathrm{CDCl}_{3}\right)$ :

High Resolution ESMS (m/z):
3295 (m), 2129 (w), 1793 (s), 1712 (s)

$\delta 5.71(\mathrm{db}, J=7.2,1 \mathrm{H}), 5.00(\mathrm{sb}, 1 \mathrm{H}), 4.73-4.68(\mathrm{~m}$, $5 \mathrm{H}), 3.31-3.15(\mathrm{~m}, 2 \mathrm{H}), 2.52-2.47$ (two triplets, $2 \mathrm{H}), 2.05-1.91(\mathrm{~m}, 2 \mathrm{H}), 1.645-1.51(\mathrm{~m}, 4 \mathrm{H})$ $\delta$ 168.5, 155.8, 155.0, 78.1, 77.6, 75.0, 74.6, 53.6, $53.0,52.5,40.1,31.3,29.2,21.8$ $\delta-40.74(\mathrm{~d}, 2 \mathrm{~F}),-45.45(\mathrm{t}, 1 \mathrm{~F}),-50.18(\mathrm{t}, 2 \mathrm{~F})$ Calculated for $\mathrm{C}_{20} \mathrm{H}_{17} \mathrm{~F}_{5} \mathrm{~N}_{2} \mathrm{O}_{6}+\mathrm{Na}$ : 499.0905 Observed: 499.0911<smiles>C#CCOC(=O)N[C@@H](CCC(=O)Oc1c(F)c(F)c(F)c(F)c1F)C(=O)Oc1c(F)c(F)c(F)c(F)c1F</smiles>

$62 \%$

Gummy solid

$[\alpha]_{\mathrm{D}}-21.00(\mathrm{c}=1.0$, ethanol)

3301 (m), 2133 (w), 1791 (s), 1716 (s)

$\delta 5.44(\mathrm{db}, J=8.4,1 \mathrm{H}), 4.91-4.90(\mathrm{~m}, 1 \mathrm{H}), 4.77-4.75$ $(\mathrm{m}, 2 \mathrm{H}), 3.01-2.83(\mathrm{~m}, 2 \mathrm{H}), 2.65-2.50(\mathrm{~m}, 2 \mathrm{H})$, 2.38-2.25 (m, $2 \mathrm{H})$

$\delta 168.3,167.8,155.0,75.9,75.2,55.7,53.38,53.0$, $29.1,27.1$

$\delta-40.73(\mathrm{~d}, 2 \mathrm{~F}),-40.99(\mathrm{~d}, 2 \mathrm{~F}),-44.80(\mathrm{t}, 1 \mathrm{~F}),-$ 45.81 (t, 1F), -49.75 (t, 2F), -50.36 (t, 2F)

Calculated for $\mathrm{C}_{21} \mathrm{H}_{9} \mathrm{~F}_{10} \mathrm{NO}_{6}+\mathrm{Na}: 584.0168$ 
Observed: 584.0159

\section{Propargyl $m$-( $N$-Poc)aminobenzoate (10)}

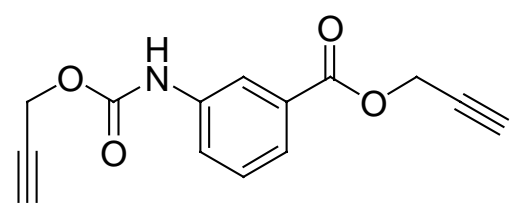

Physical Appearance:

Melting Point:

FTIR (KBr):

${ }^{1} \mathrm{H}$ NMR (300 MHz, $\left.\mathrm{CDCl}_{3}\right)$ :

${ }^{13} \mathrm{C}$ NMR (75 MHz, $\left.\mathrm{CDCl}_{3}\right)$ :

High Resolution ESMS (m/z):
White solid

$146{ }^{\circ} \mathrm{C}$

3338 (m), $2129(\mathrm{w}), 1723$ (s), 1704 (s)

$\delta 9.35(\mathrm{sb}, 1 \mathrm{H}), 8.19-8.17(\mathrm{~m}, 1 \mathrm{H}), 7.78-7.69(\mathrm{~m}$, 2H), 7.38-7.33 (m, 1H), $4.90(\mathrm{~d}, J=2.7,2 \mathrm{H}), 4.78$

$(\mathrm{d}, J=2.7,2 \mathrm{H}), 2.61-2.58(\mathrm{~m}, 2 \mathrm{H})$

$\delta 165.0,152.5,138.6,129.4,128.4,123.7,123.1$,

119.2, 77.6, 77.2, 74.8, 74.6, 51.9

Calculated for $\mathrm{C}_{14} \mathrm{H}_{11} \mathrm{NO}_{4}+\mathrm{Na}: 280.0586$

Observed: 280.0593 


\section{Compound 4a}<smiles>CC(C)(C)OC(=O)N1CCC[C@H]1C(=O)Oc1c(F)c(F)c(F)c(F)c1F</smiles>

${ }^{19}$ F NMR $\left(282 \mathrm{MHz}, \mathrm{CDCl}_{3}\right)$

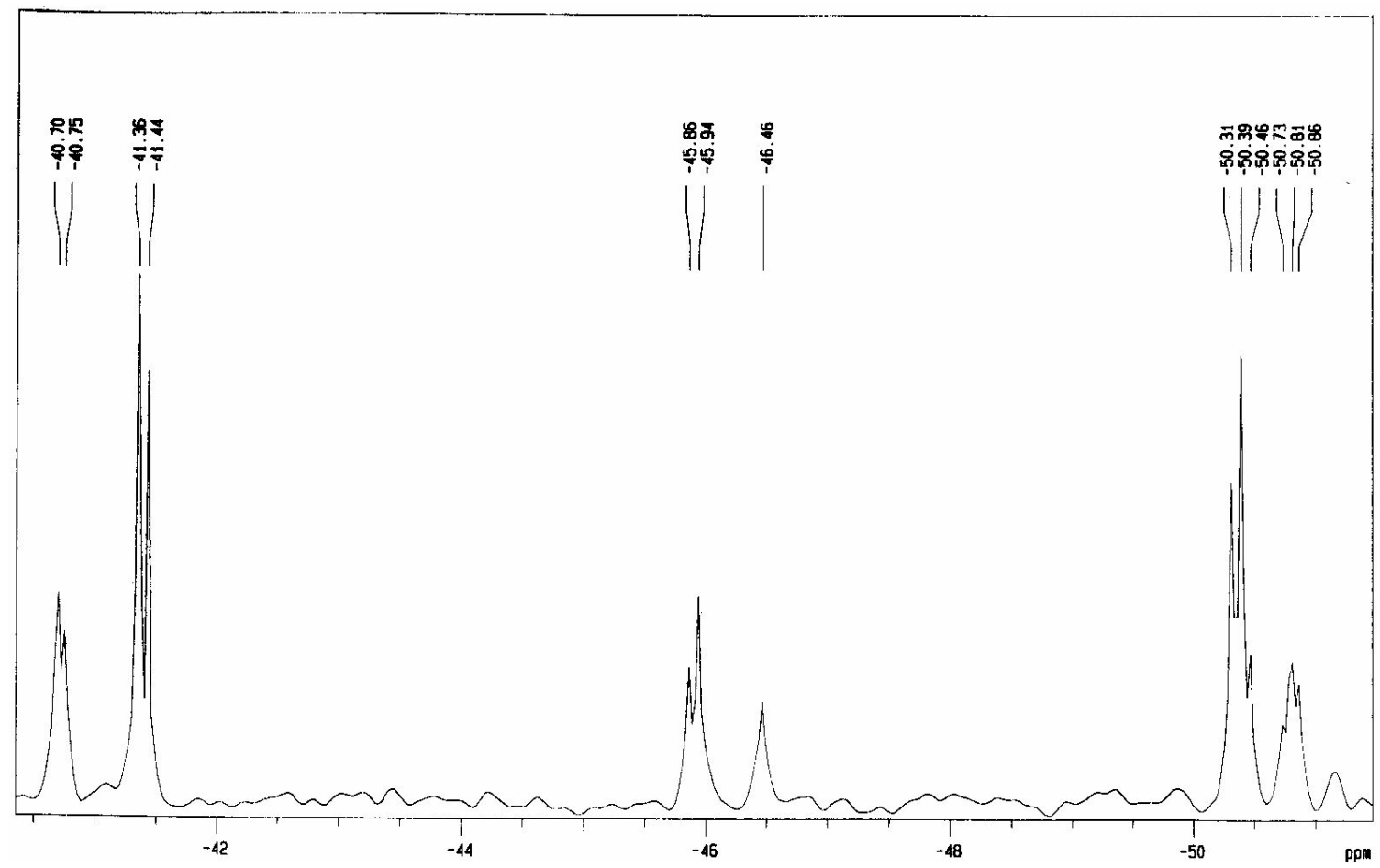




\section{Compound 4a}

${ }^{1} \mathrm{H}$ NMR (300 MHz, $\mathrm{CDCl}_{3}$ )
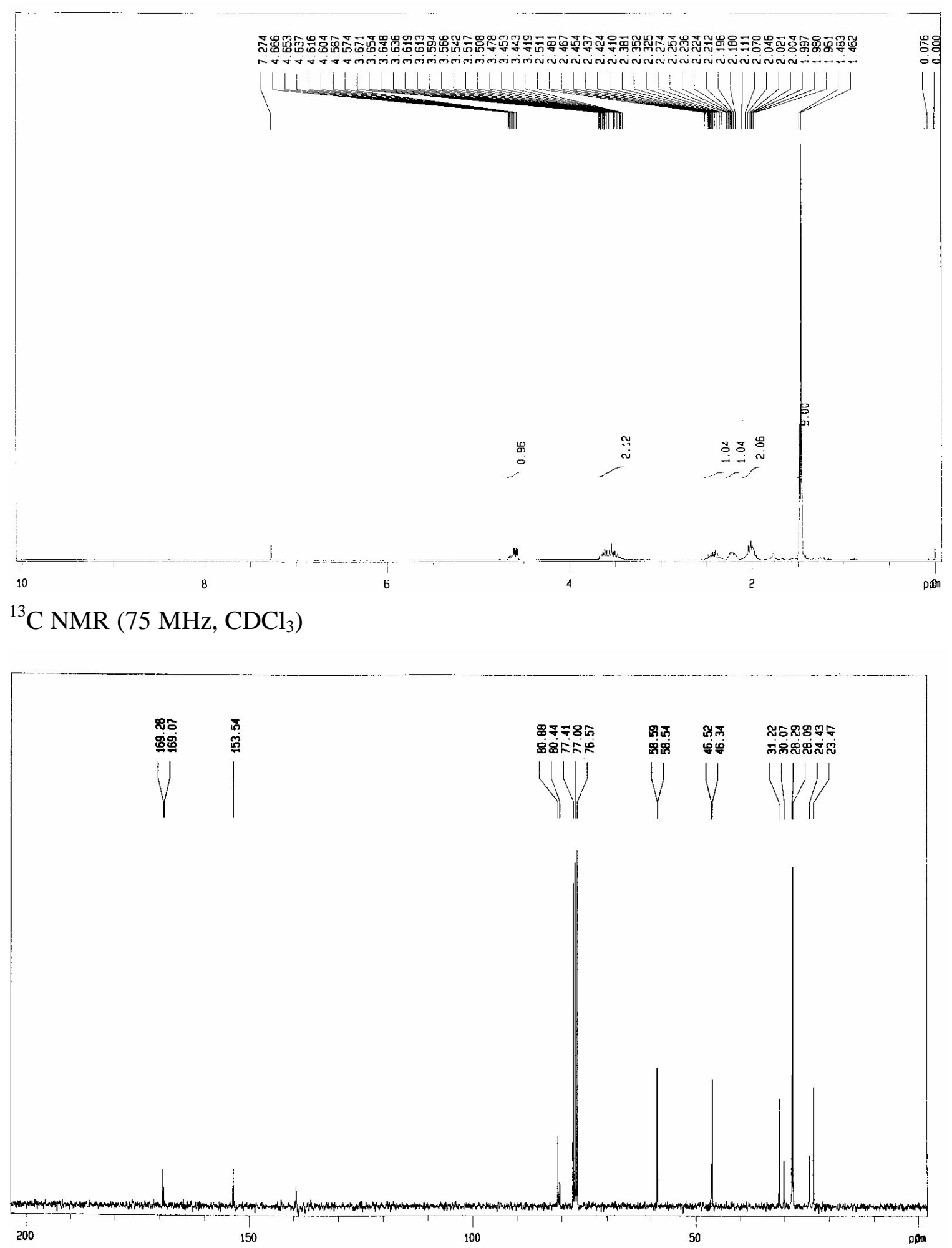


\section{Compound 4b}<smiles>CC(C)C[C@H](NC(=O)OC(C)(C)C)C(=O)Oc1c(F)c(F)c(F)c(F)c1F</smiles>

${ }^{19} \mathrm{~F}$ NMR (282 MHz, $\mathrm{CDCl}_{3}$ )

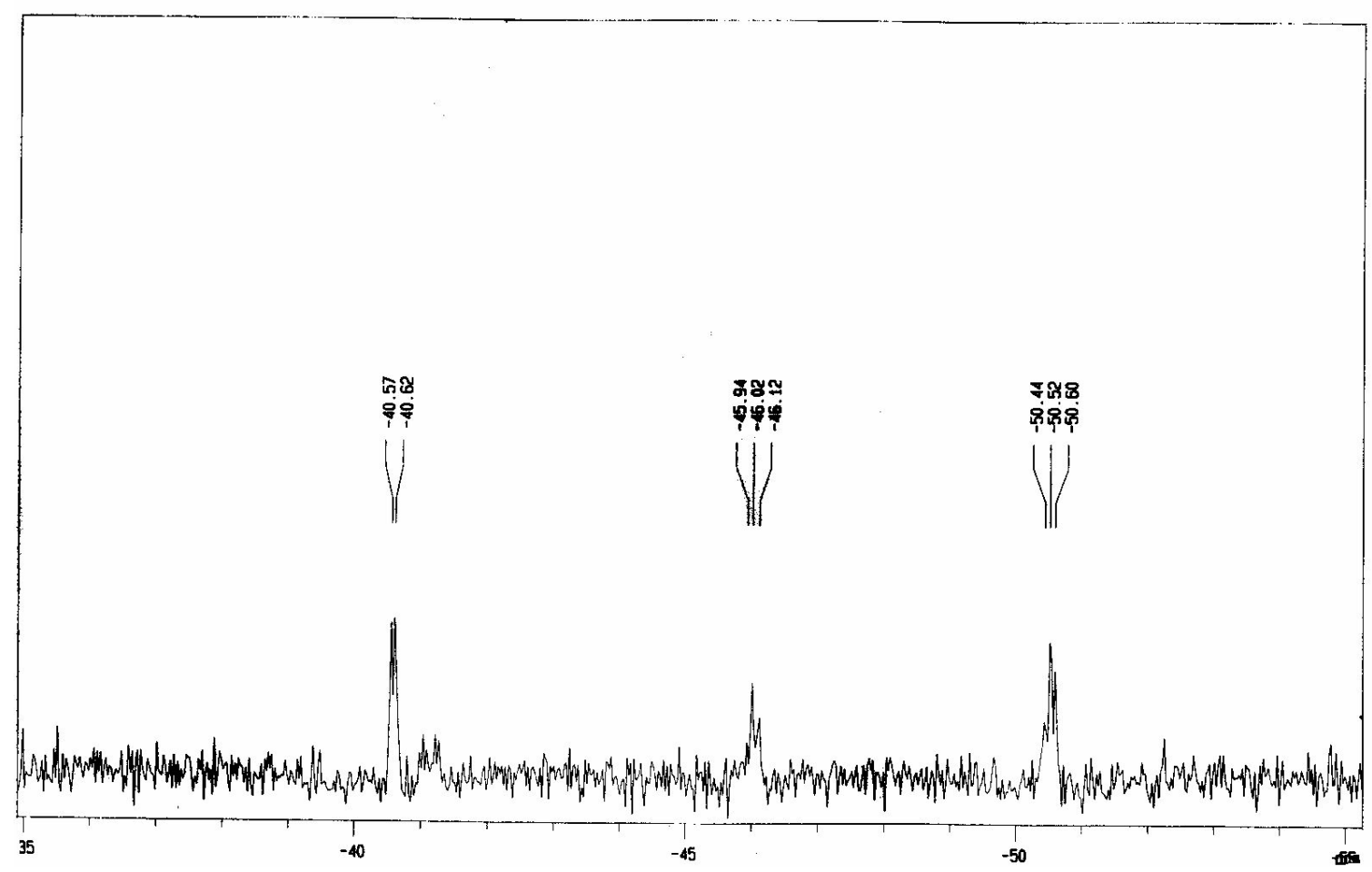




\section{Compound 4b}

${ }^{1} \mathrm{H}$ NMR $\left(300 \mathrm{MHz}, \mathrm{CDCl}_{3}\right.$ )
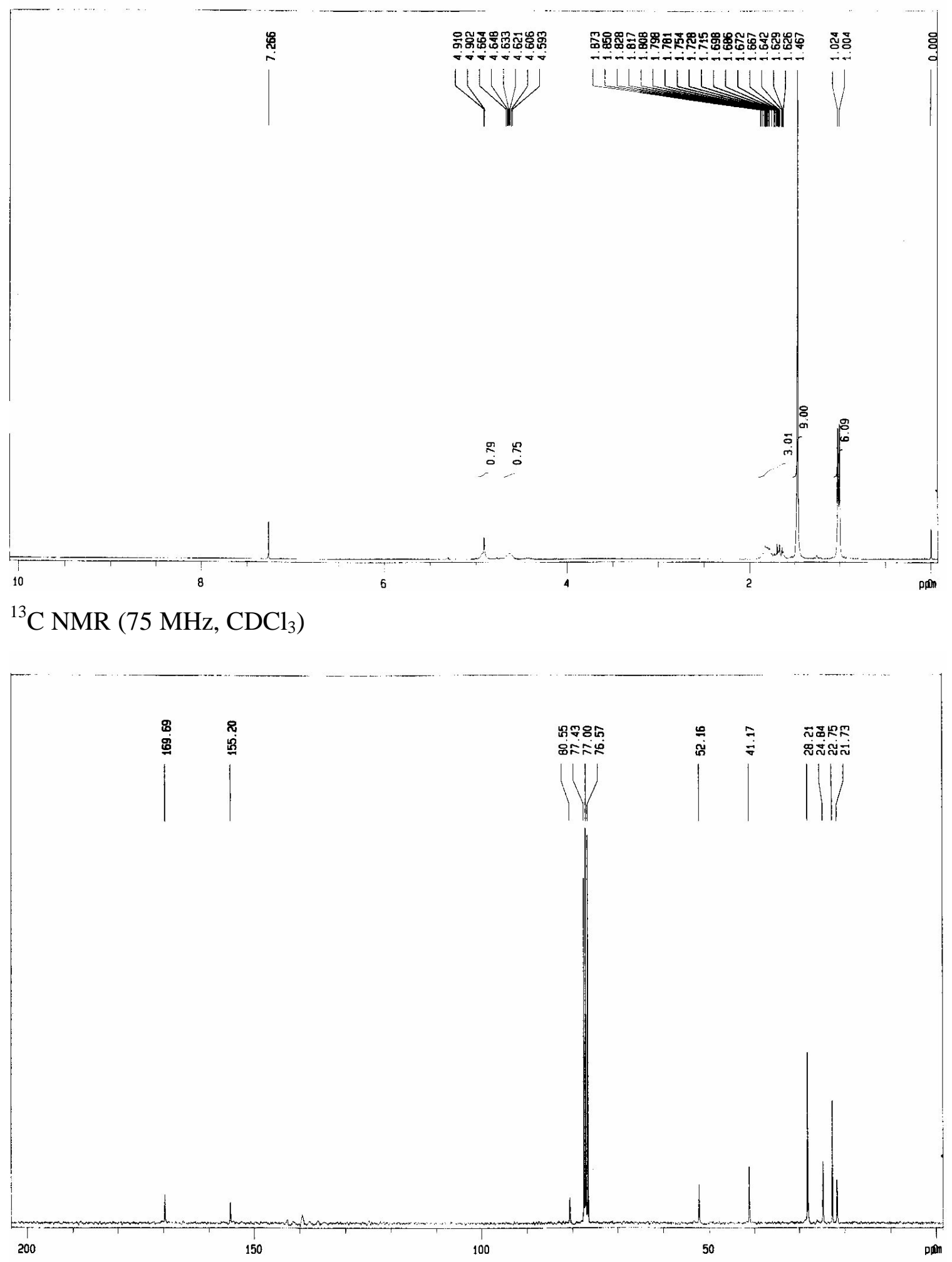


\section{Compound 4c}<smiles>O=C(N[C@@H](Cc1ccccc1)C(=O)Oc1c(F)c(F)c(F)c(F)c1F)OCc1ccccc1</smiles>

${ }^{19} \mathrm{~F}$ NMR (282 MHz, $\mathrm{CDCl}_{3}$ )

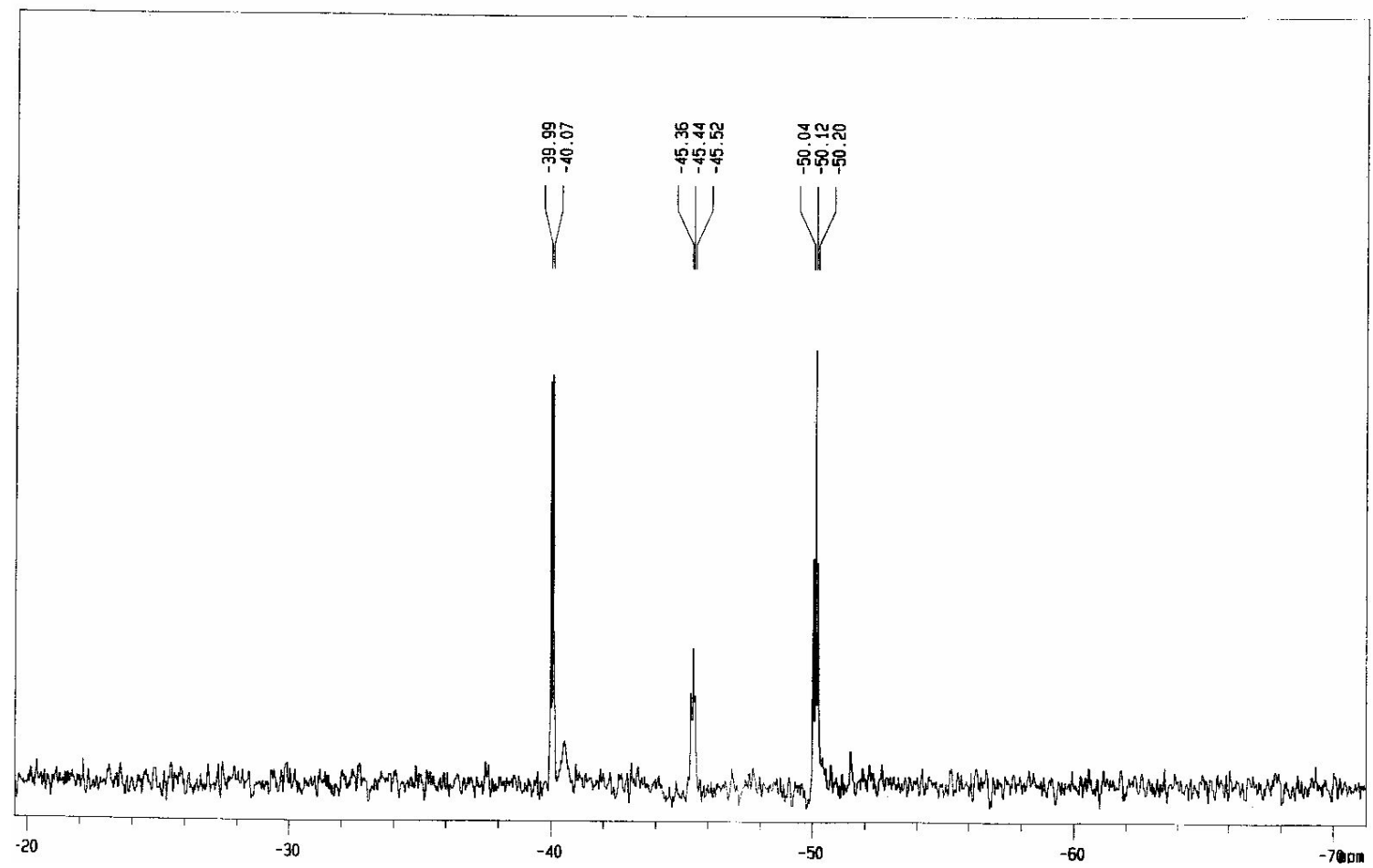




\section{Compound 4c}

${ }^{1} \mathrm{H}$ NMR (300 MHz, $\mathrm{CDCl}_{3}$ )

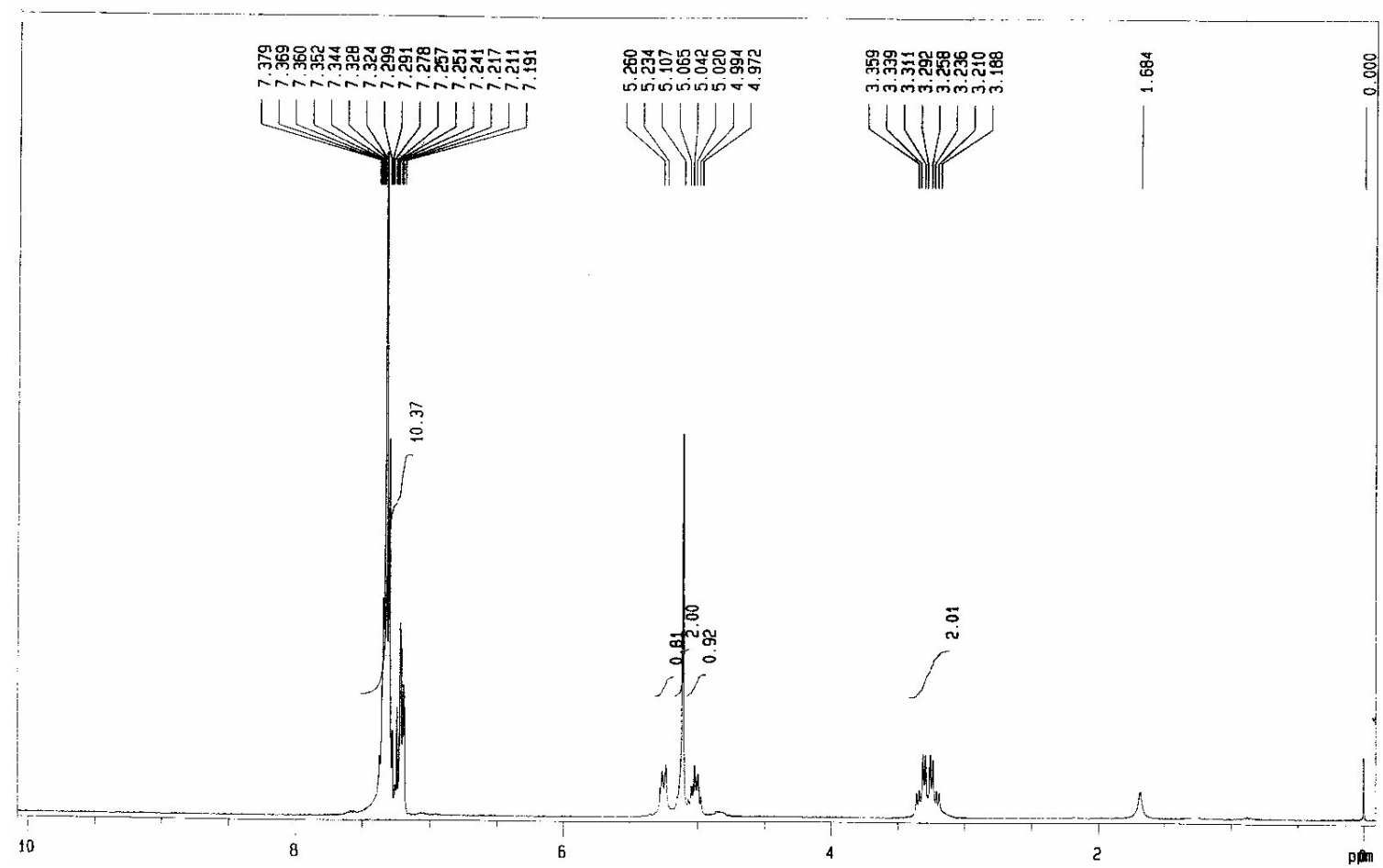

${ }^{13} \mathrm{C}$ NMR $\left(75 \mathrm{MHz}, \mathrm{CDCl}_{3}\right)$

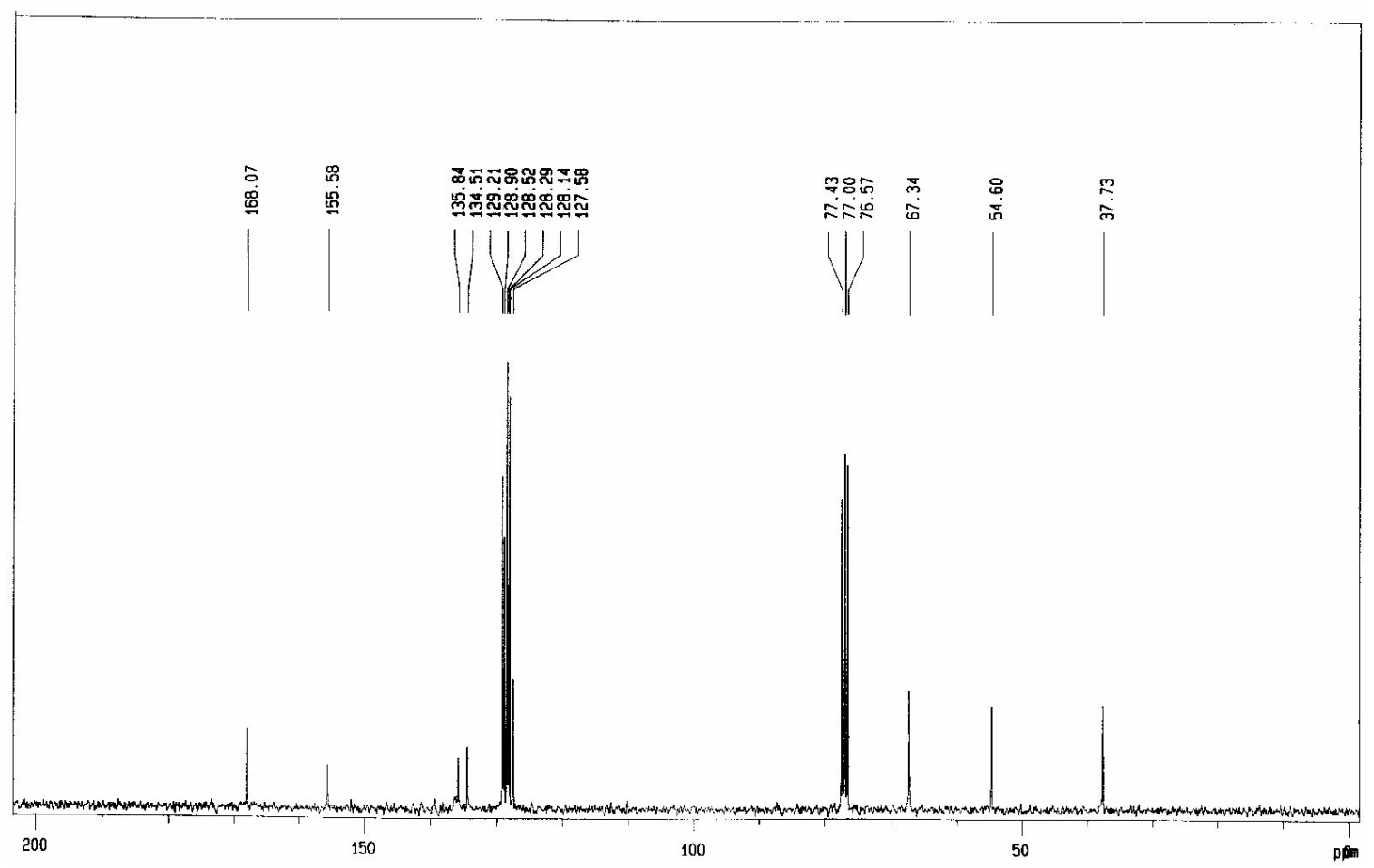




\section{Compound 6a}<smiles>C#CCOC(=O)Nc1cccc(C(=O)Oc2c(F)c(F)c(F)c(F)c2F)c1</smiles>

${ }^{19}$ F NMR $\left(282 \mathrm{MHz}, \mathrm{CDCl}_{3}\right)$

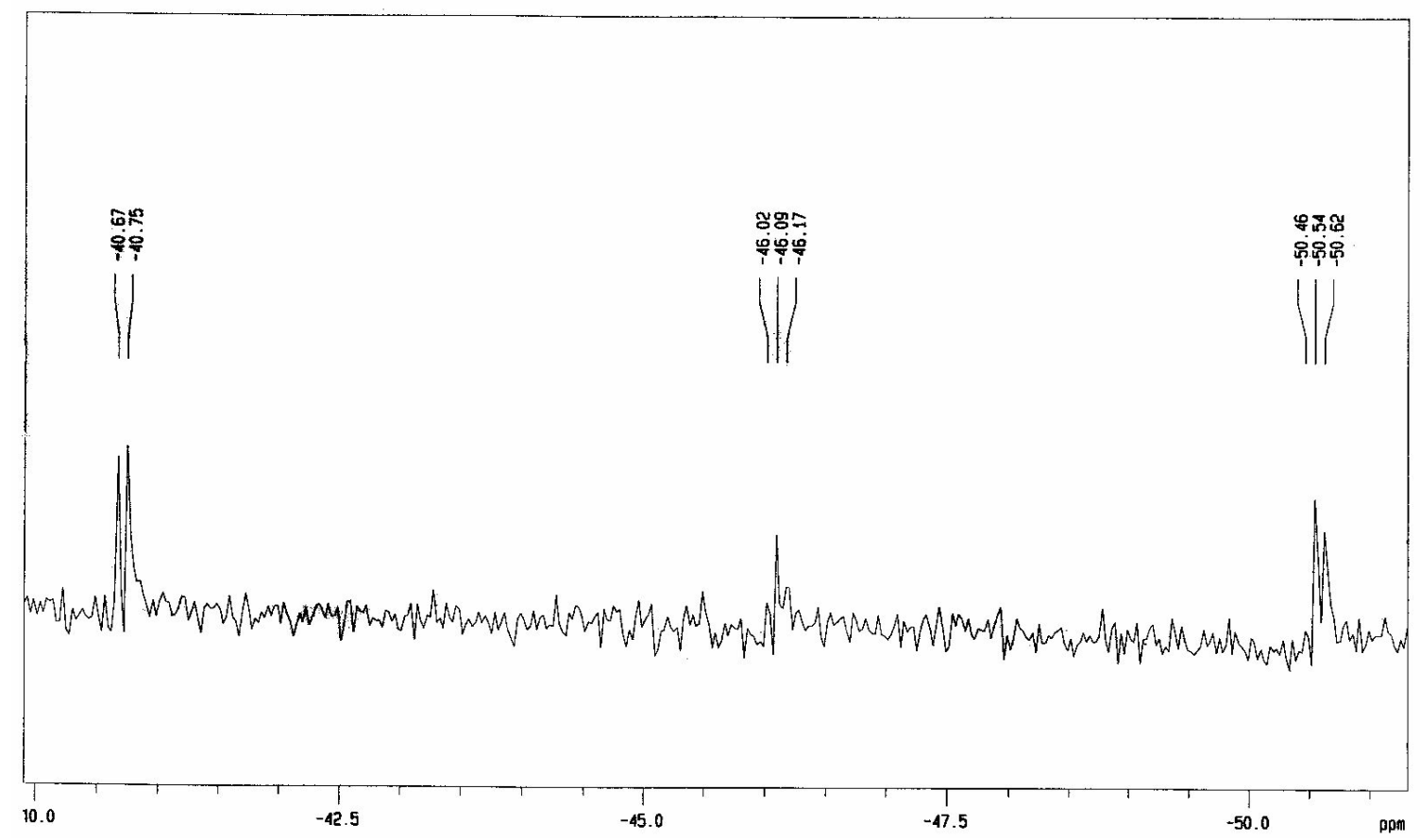




\section{Compound 6a}

${ }^{1} \mathrm{H}$ NMR $\left(300 \mathrm{MHz}, \mathrm{CDCl}_{3}\right.$ )

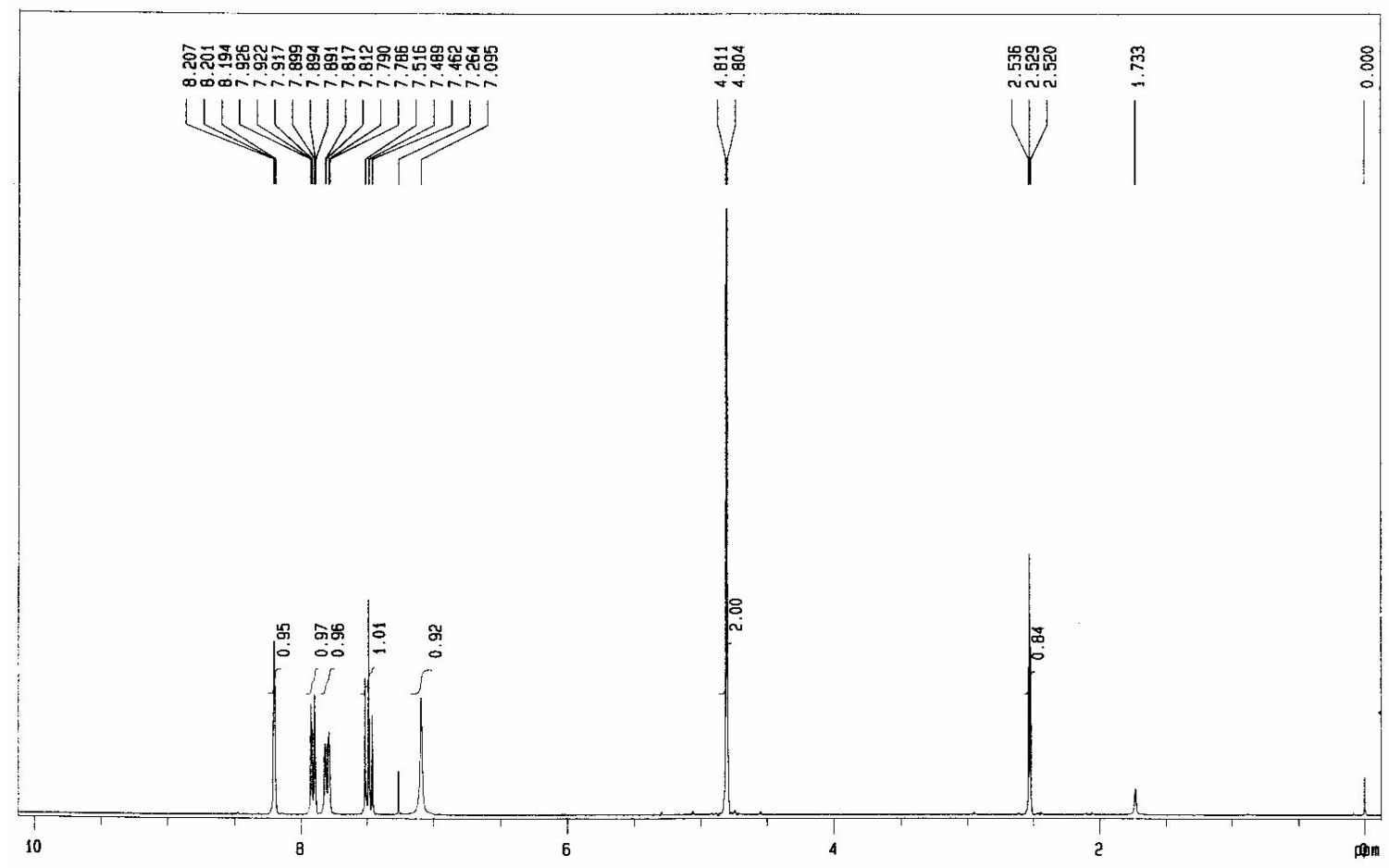

${ }^{13} \mathrm{C}$ NMR $\left(75 \mathrm{MHz}, \mathrm{CDCl}_{3}\right.$ )

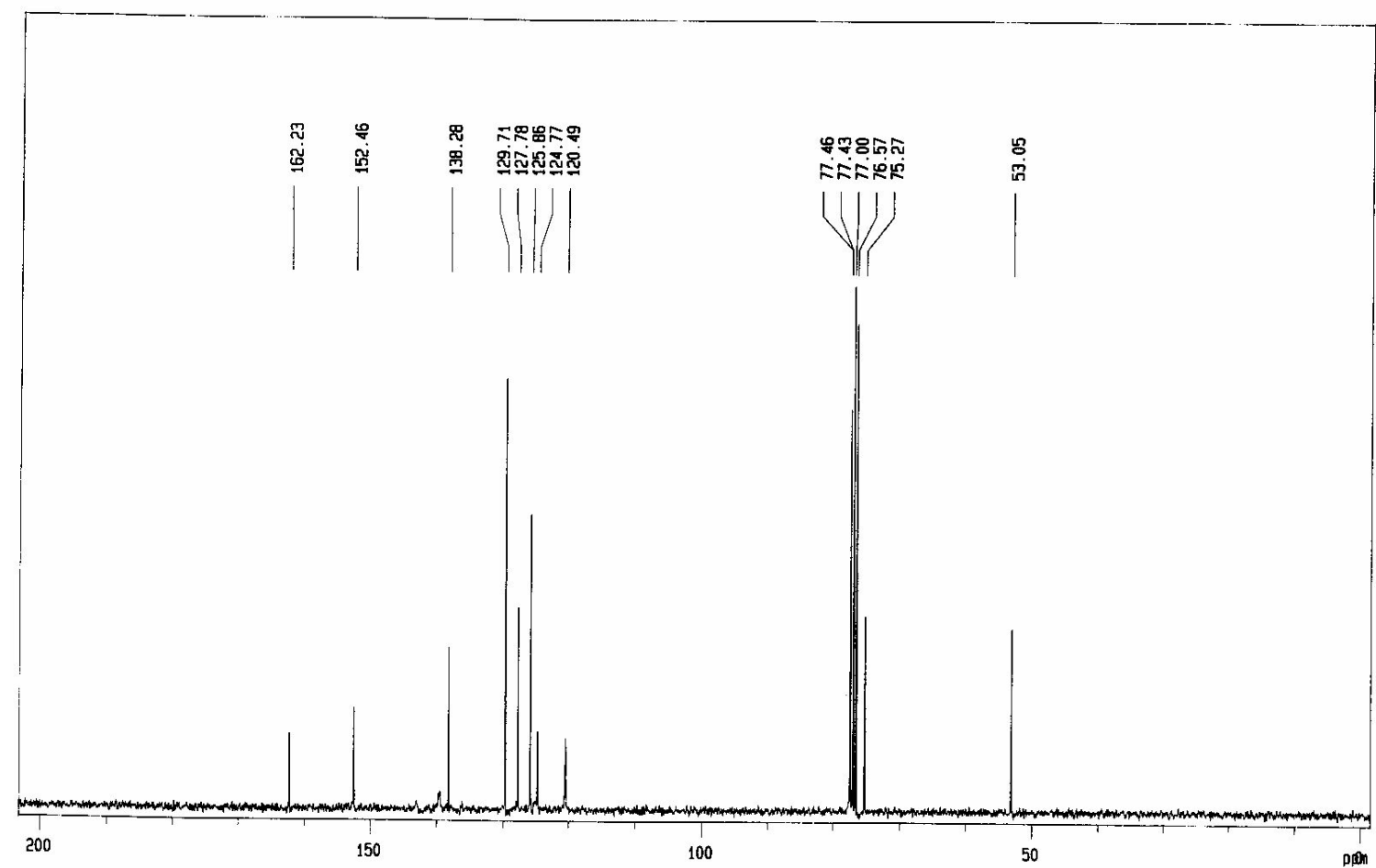




\section{Compound 6b}

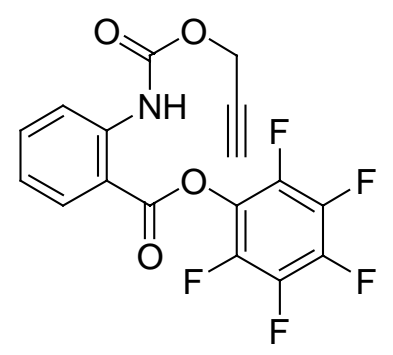

${ }^{19} \mathrm{~F}$ NMR $\left(282 \mathrm{MHz}, \mathrm{CDCl}_{3}\right)$

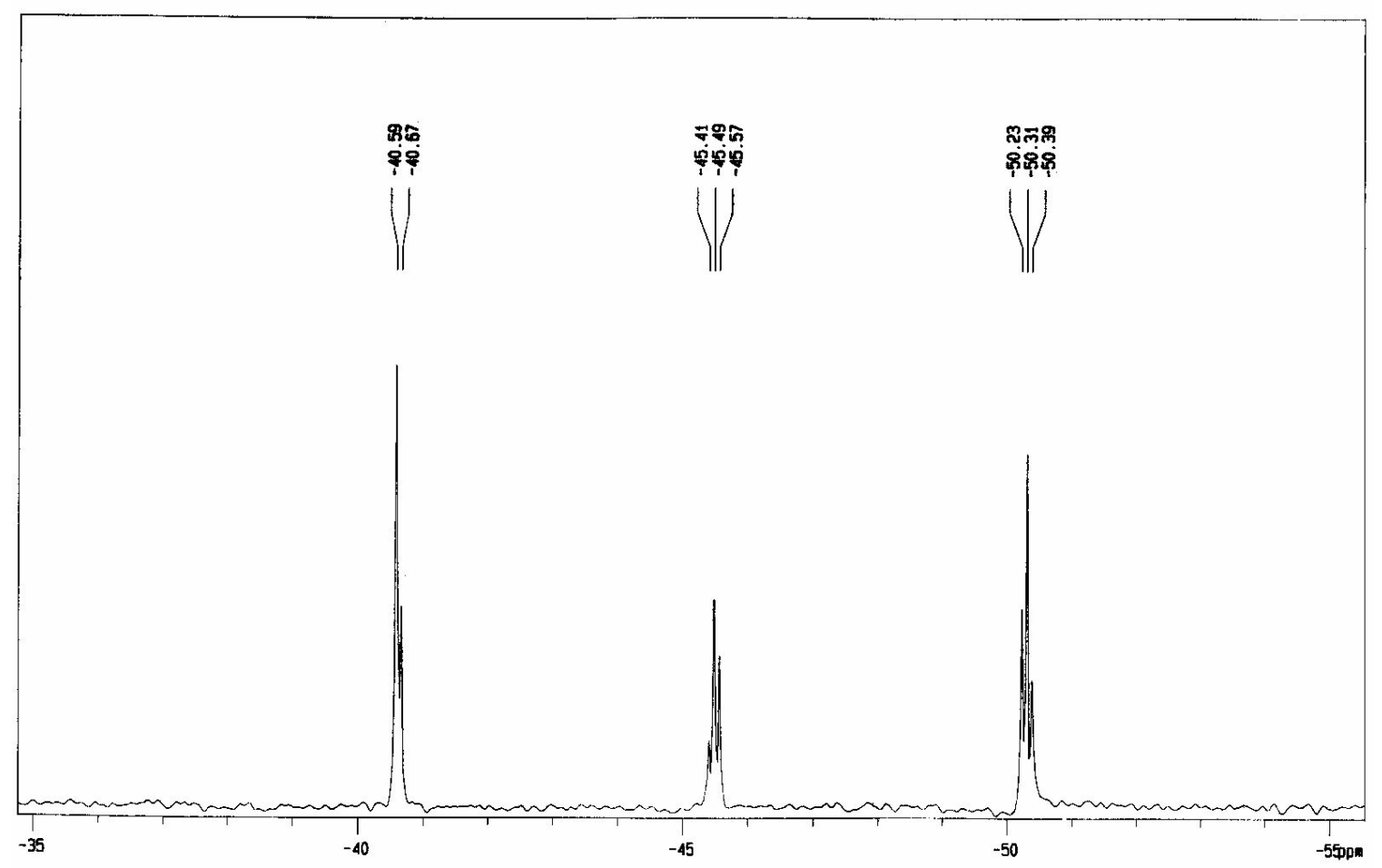




\section{Compound 6b}

${ }^{1} \mathrm{H}$ NMR (300 MHz, $\mathrm{CDCl}_{3}$ )

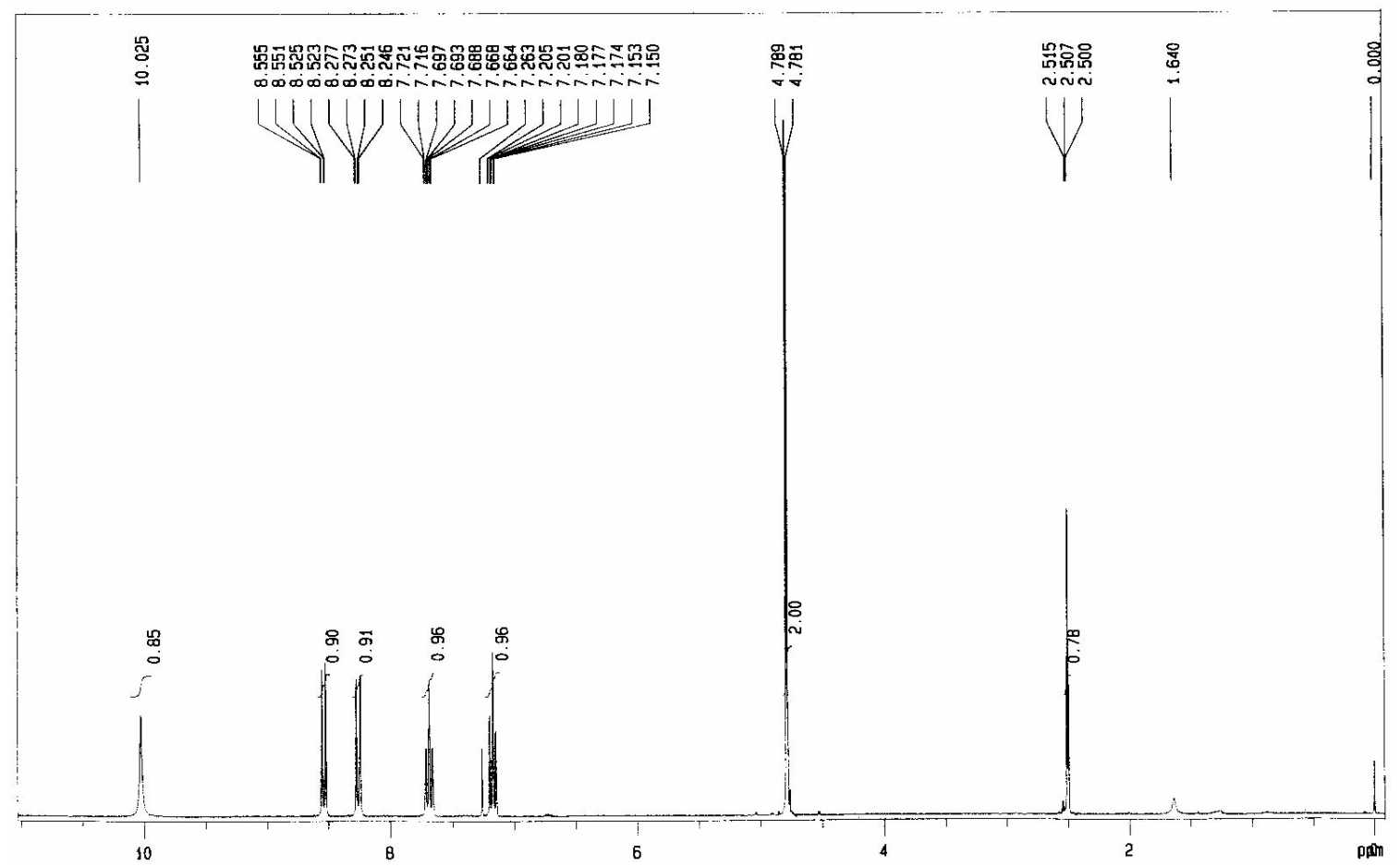

${ }^{13} \mathrm{C}$ NMR $\left(75 \mathrm{MHz}, \mathrm{CDCl}_{3}\right.$ )

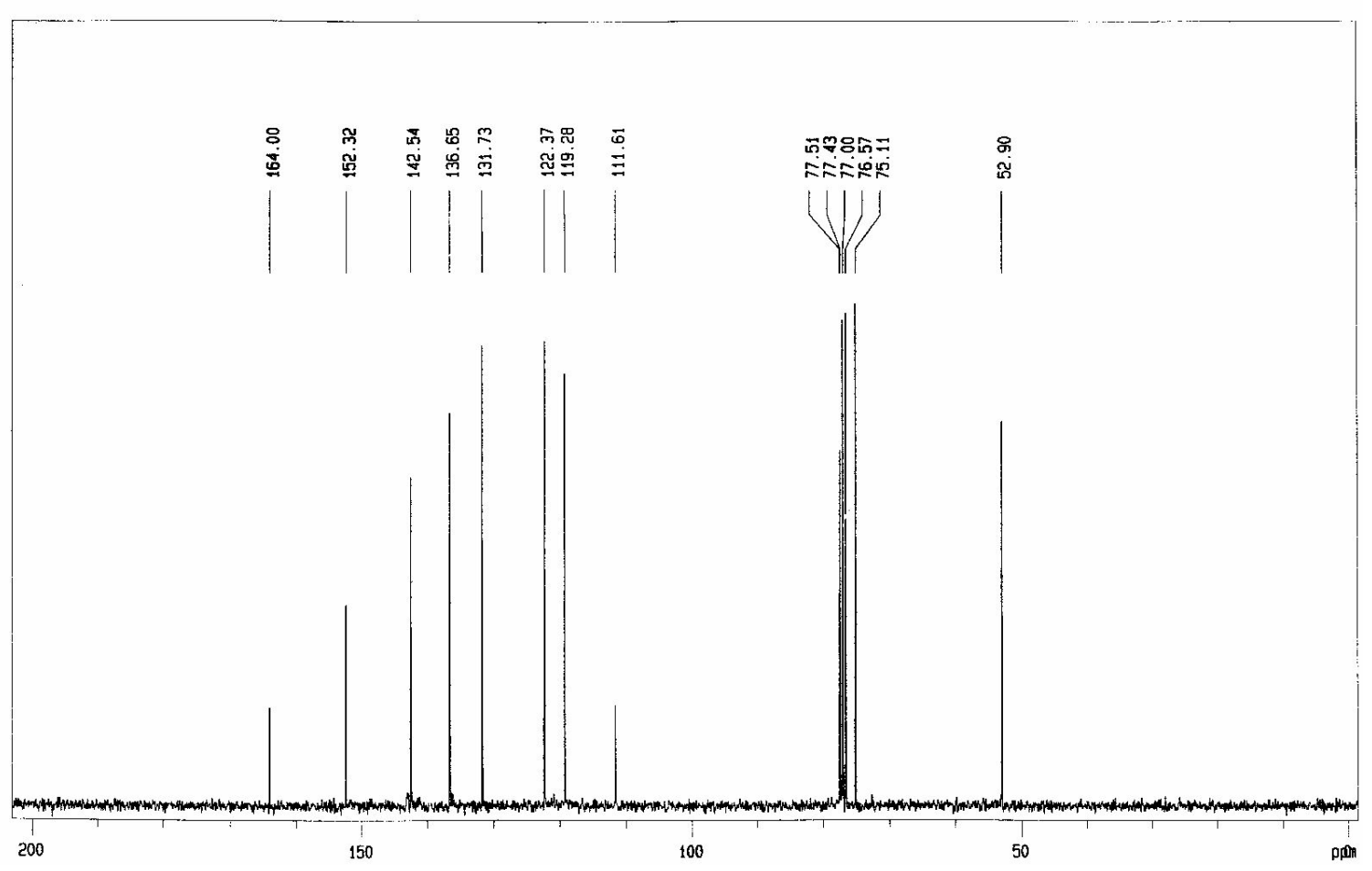




\section{Compound 6c}<smiles>C#CCOC(=O)NC(CC(C)C)C(=O)Oc1c(F)c(F)c(F)c(F)c1F</smiles>

${ }^{19} \mathrm{~F}$ NMR $\left(282 \mathrm{MHz}, \mathrm{CDCl}_{3}\right)$

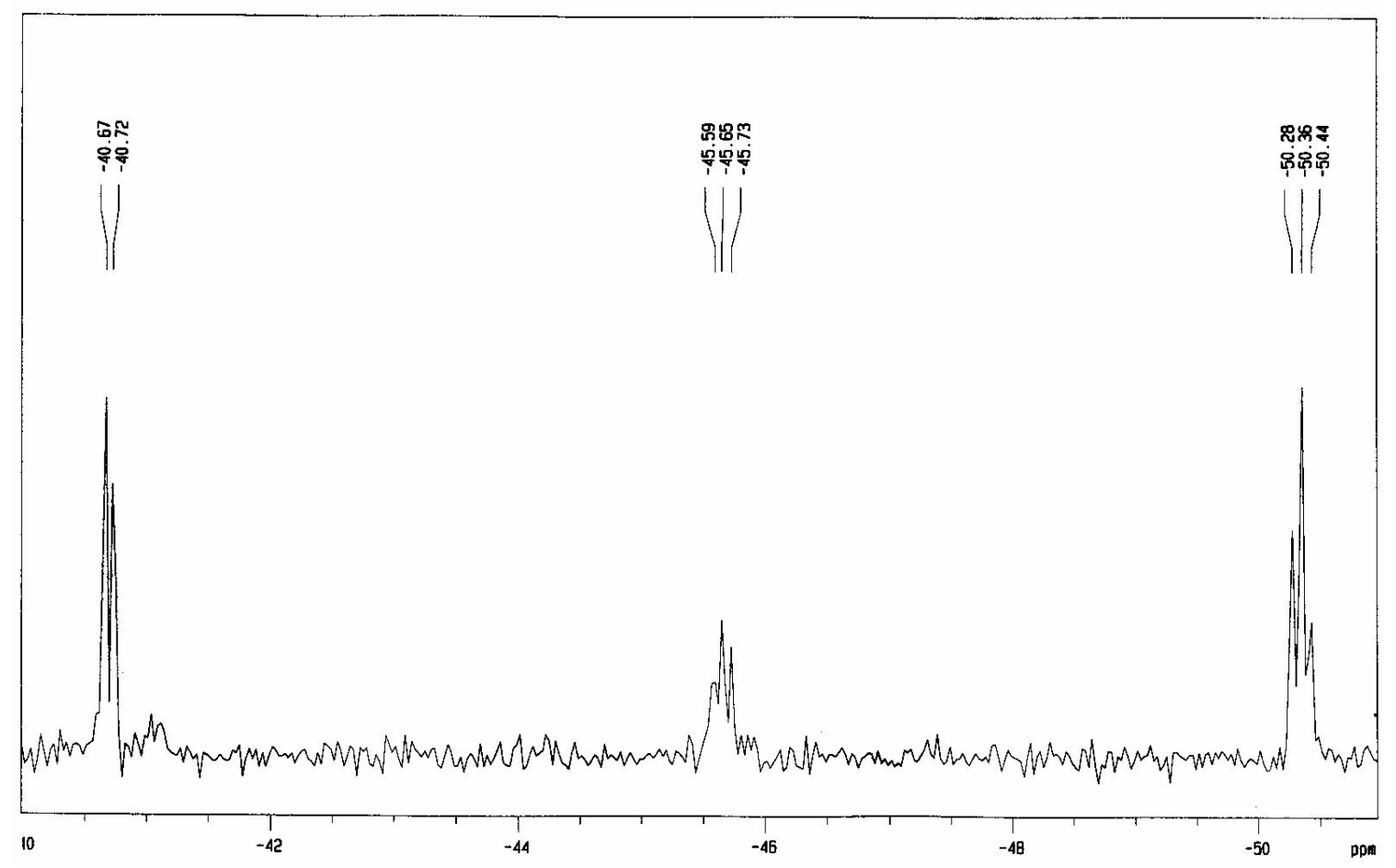




\section{Compound 6c}

${ }^{1} \mathrm{H}$ NMR (300 MHz, $\mathrm{CDCl}_{3}$ )

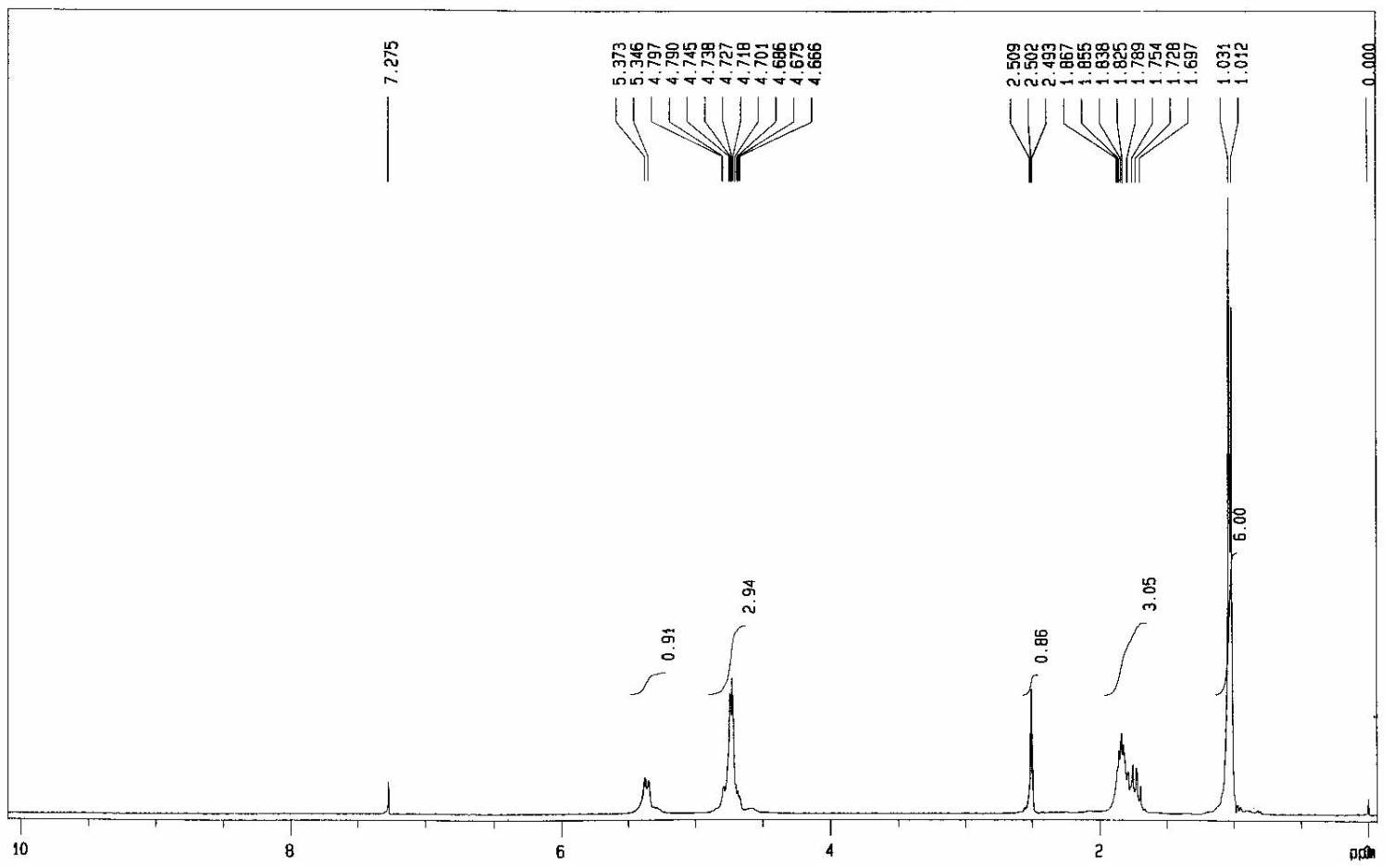

${ }^{13} \mathrm{C}$ NMR $\left(75 \mathrm{MHz}, \mathrm{CDCl}_{3}\right.$ )

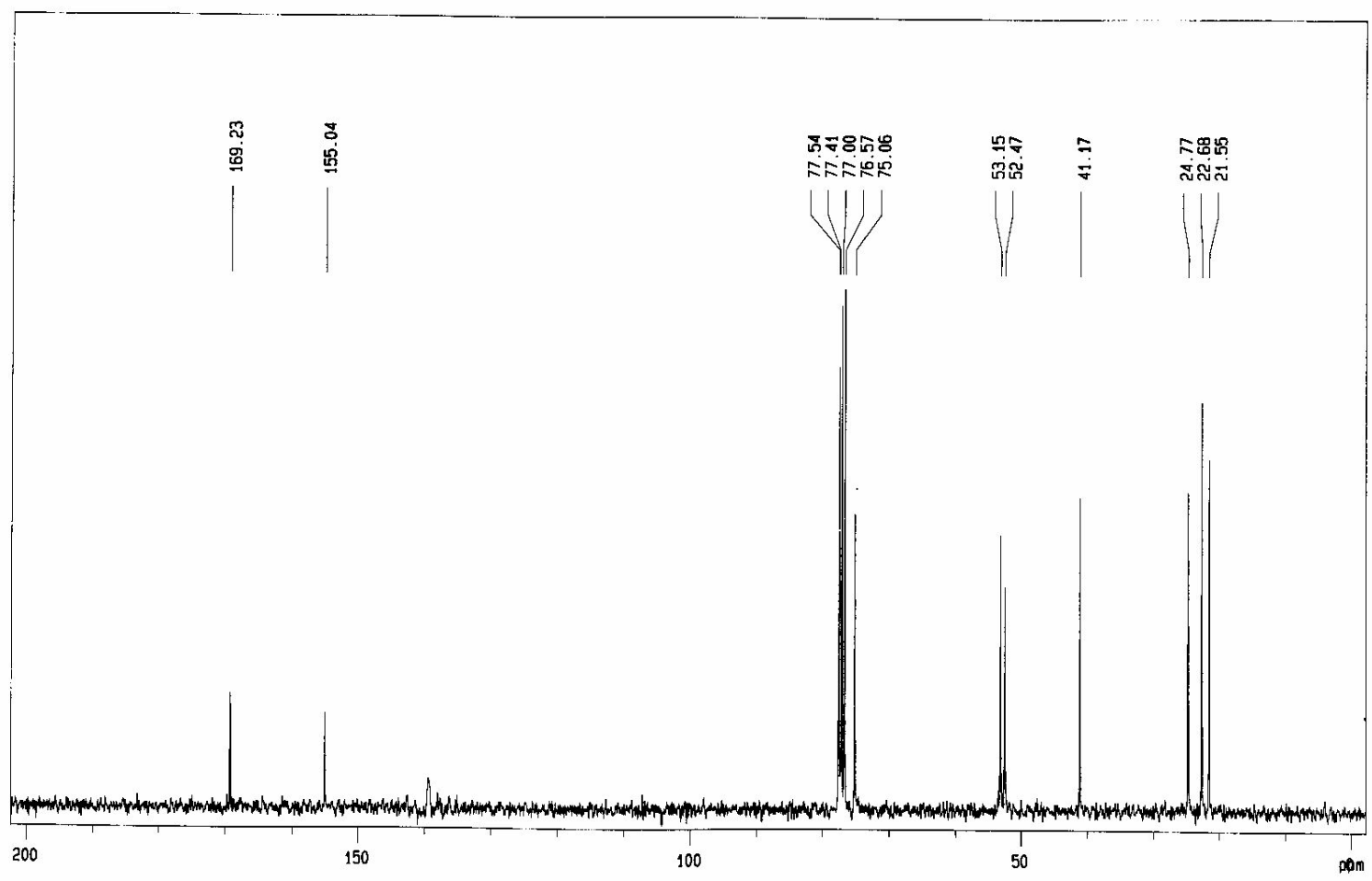




\section{Compound 6d}

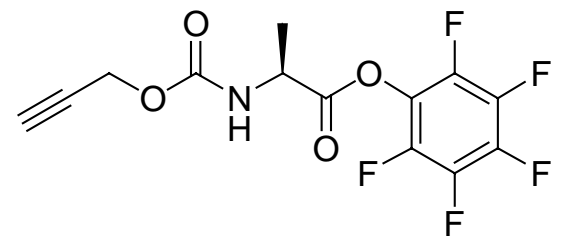

$\left.{ }^{19} \mathrm{~F} \mathrm{NMR} \mathrm{(282} \mathrm{MHz,} \mathrm{CDCl}_{3}\right)$

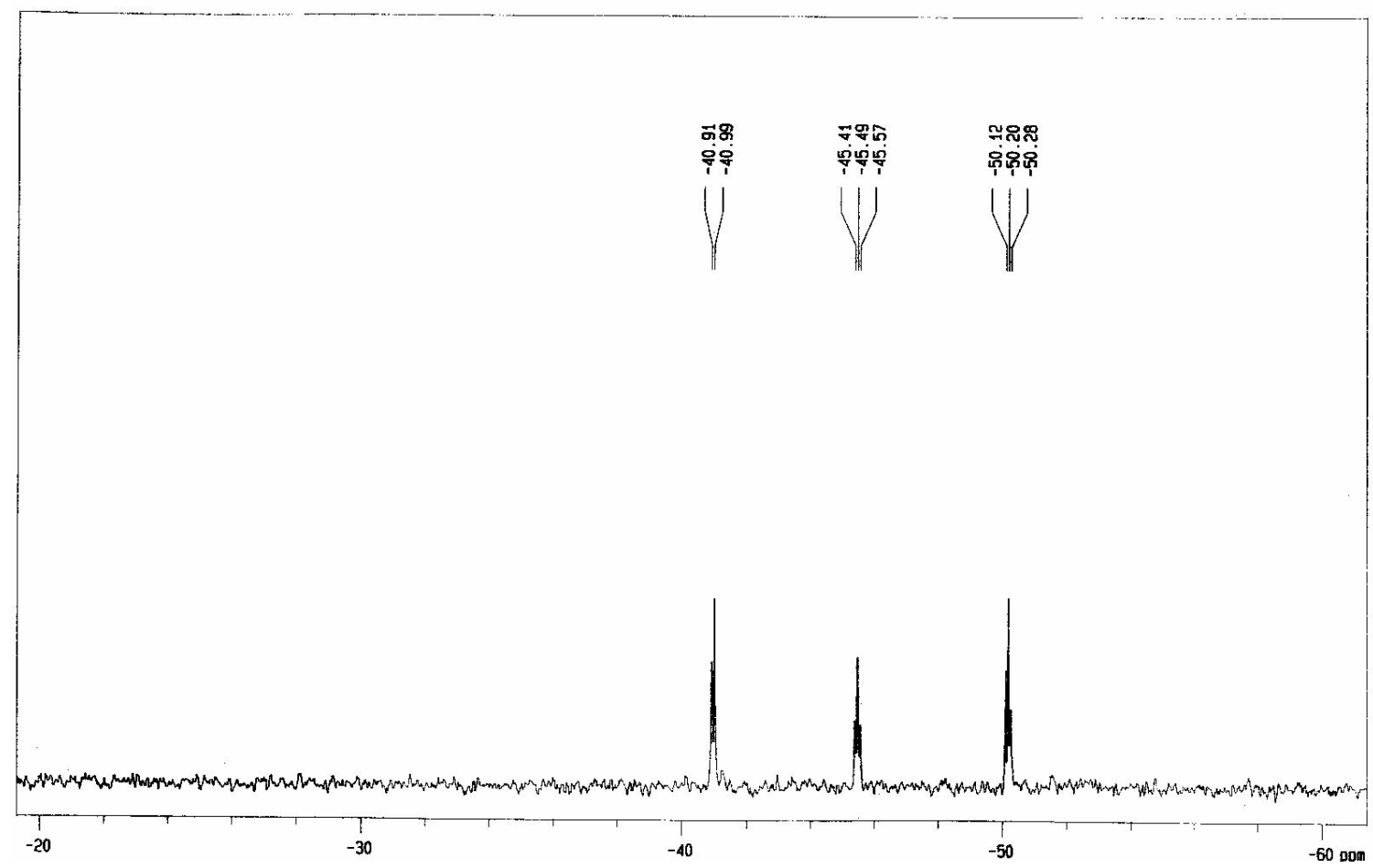




\section{Compound 6d}

${ }^{1} \mathrm{H}$ NMR $\left(300 \mathrm{MHz}, \mathrm{CDCl}_{3}\right.$ )
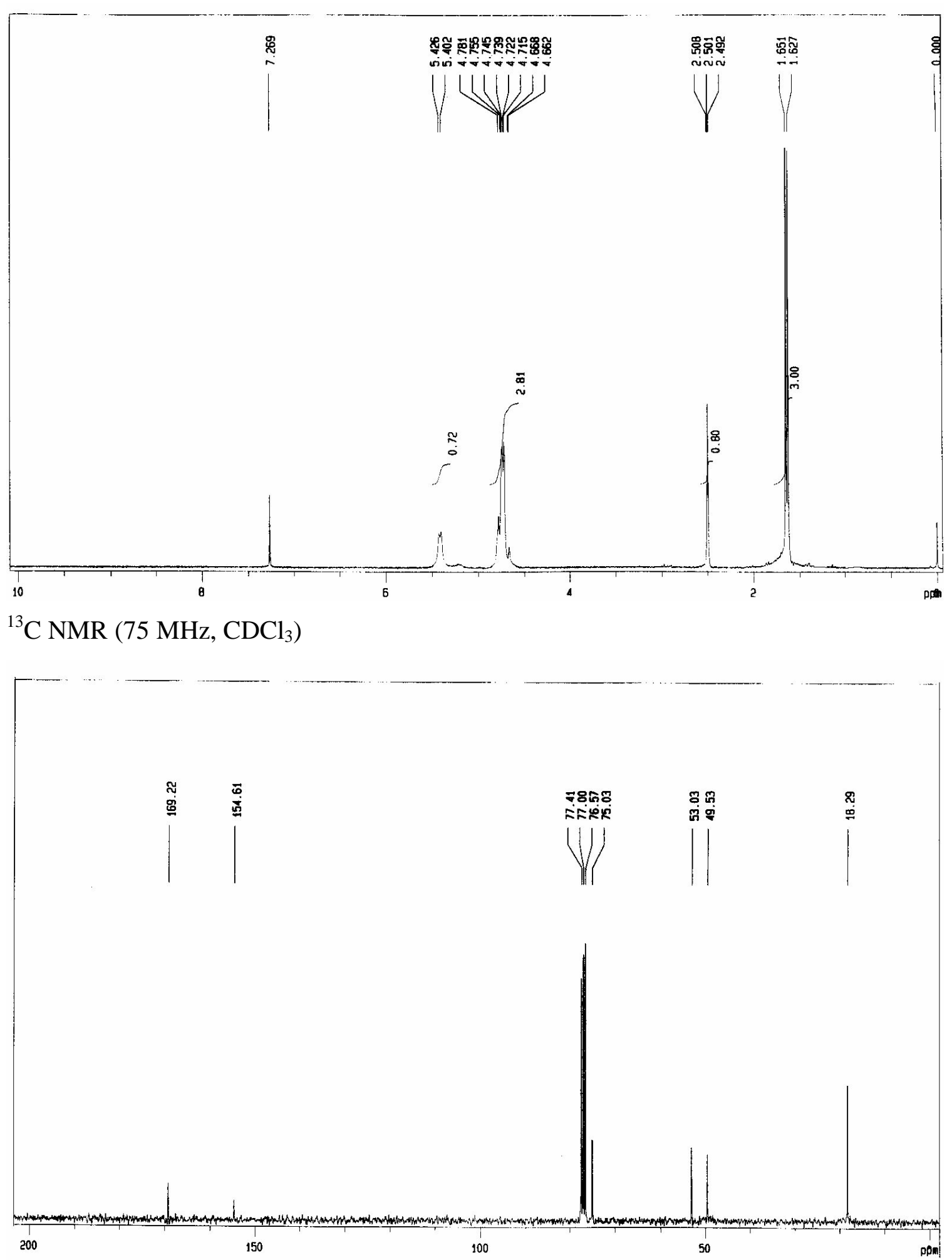


\section{Compound 6e}<smiles>C#CCOC(=O)NC(C(=O)Oc1c(F)c(F)c(F)c(F)c1F)C(C)C</smiles>

${ }^{19}$ F NMR (282 MHz, $\mathrm{CDCl}_{3}$ )

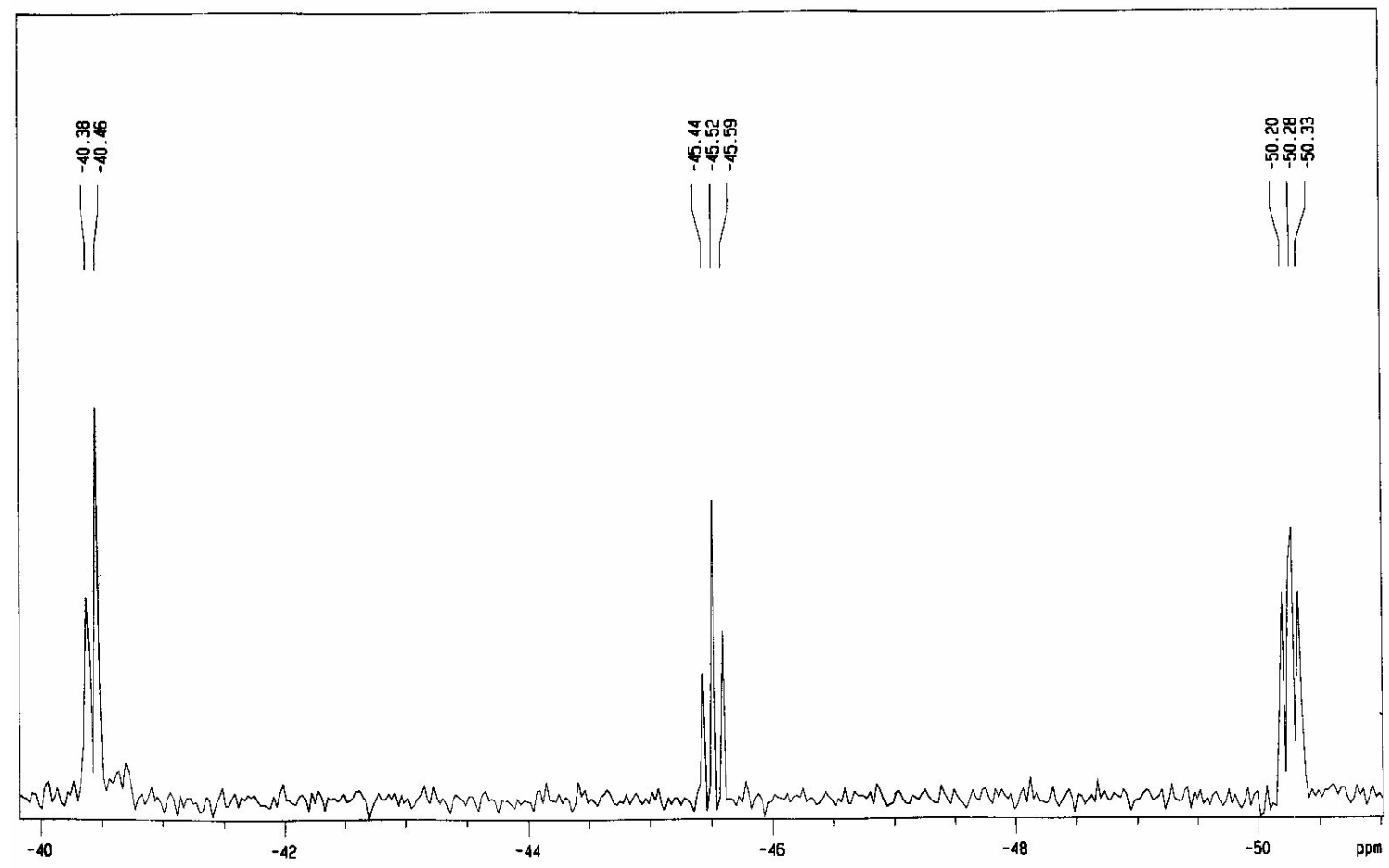




\section{Compound 6e}

${ }^{1} \mathrm{H}$ NMR $\left(300 \mathrm{MHz}, \mathrm{CDCl}_{3}\right.$ )

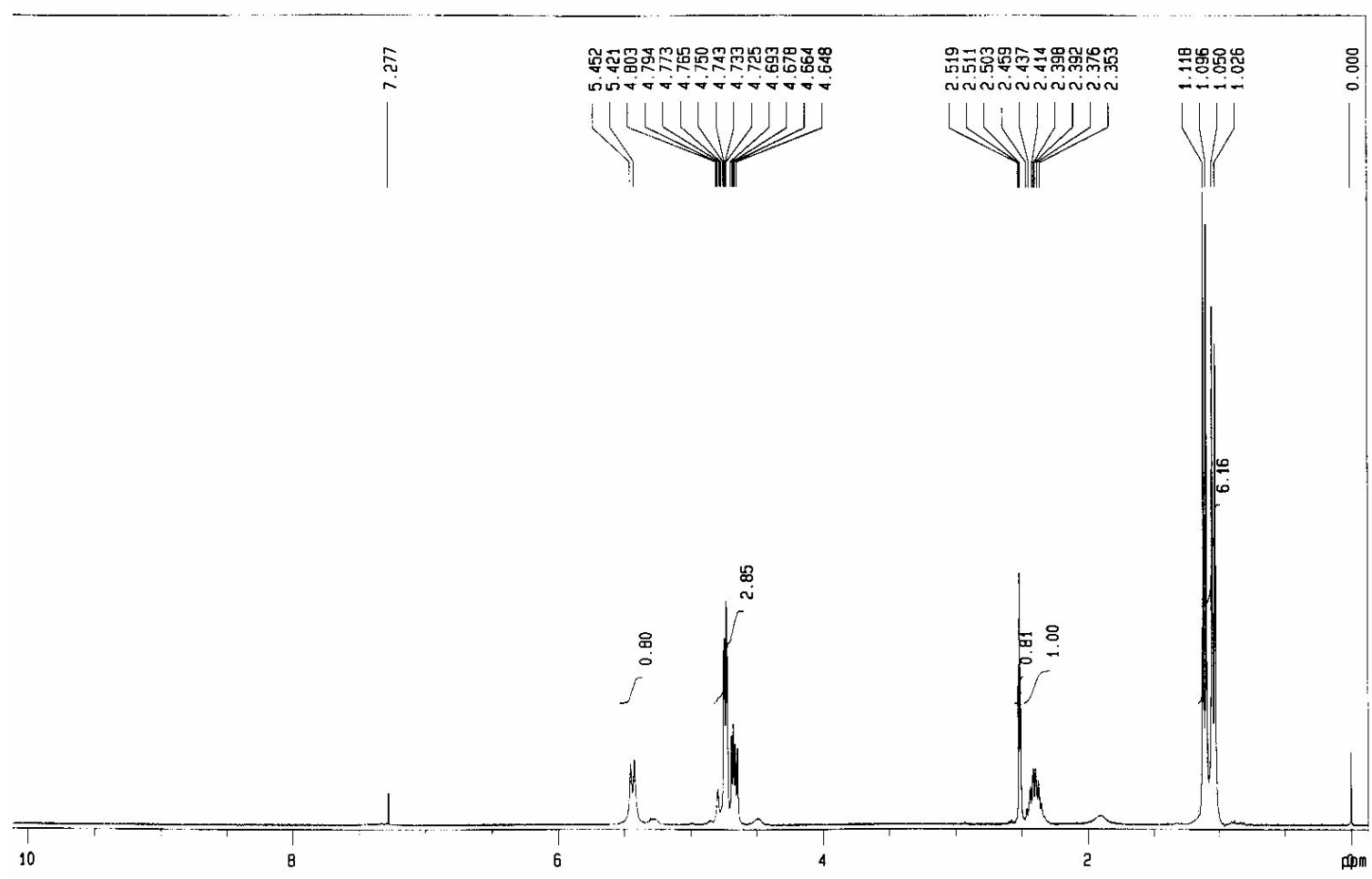

${ }^{13} \mathrm{C}$ NMR $\left(75 \mathrm{MHz}, \mathrm{CDCl}_{3}\right)$

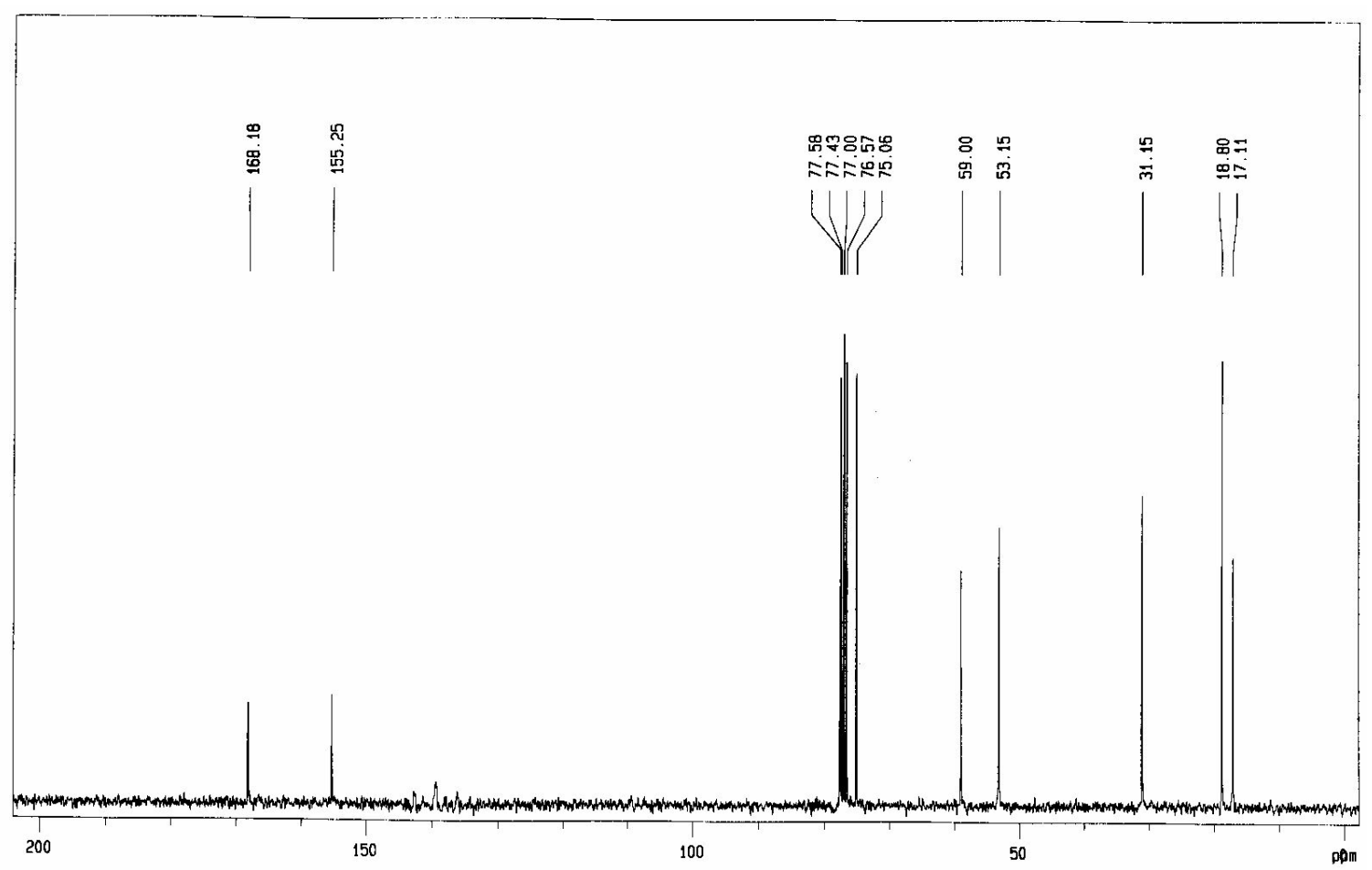




\section{Compound 6f}<smiles>C#CCOC(=O)NC(C(=O)Oc1c(F)c(F)c(F)c(F)c1F)C(C)CC</smiles>

$\left.{ }^{19} \mathrm{~F} \mathrm{NMR} \mathrm{(282} \mathrm{MHz,} \mathrm{CDCl}_{3}\right)$

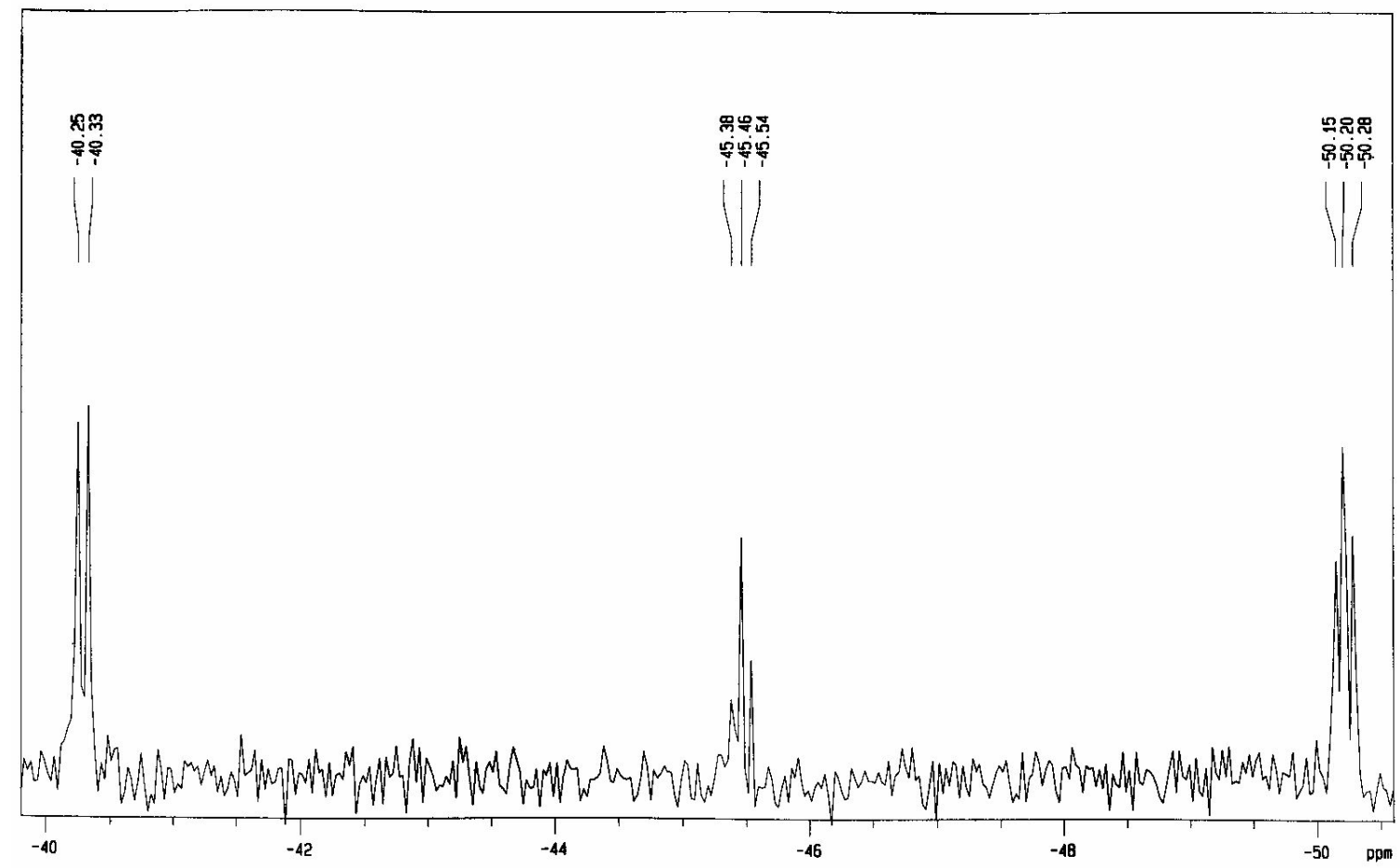




\section{Compound 6f}

${ }^{1} \mathrm{H}$ NMR (300 MHz, $\mathrm{CDCl}_{3}$ )

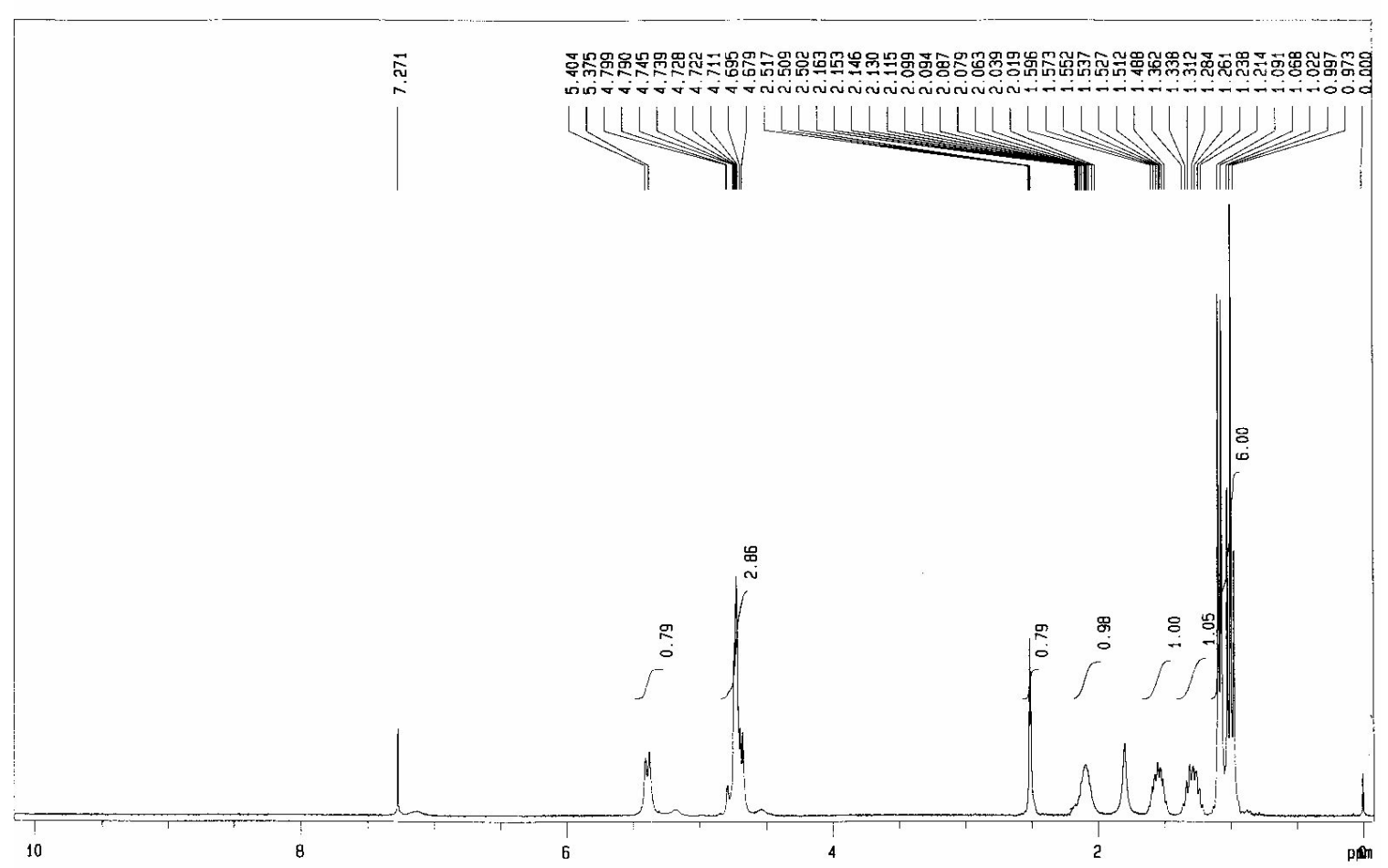

${ }^{13} \mathrm{C}$ NMR $\left(75 \mathrm{MHz}, \mathrm{CDCl}_{3}\right.$ )

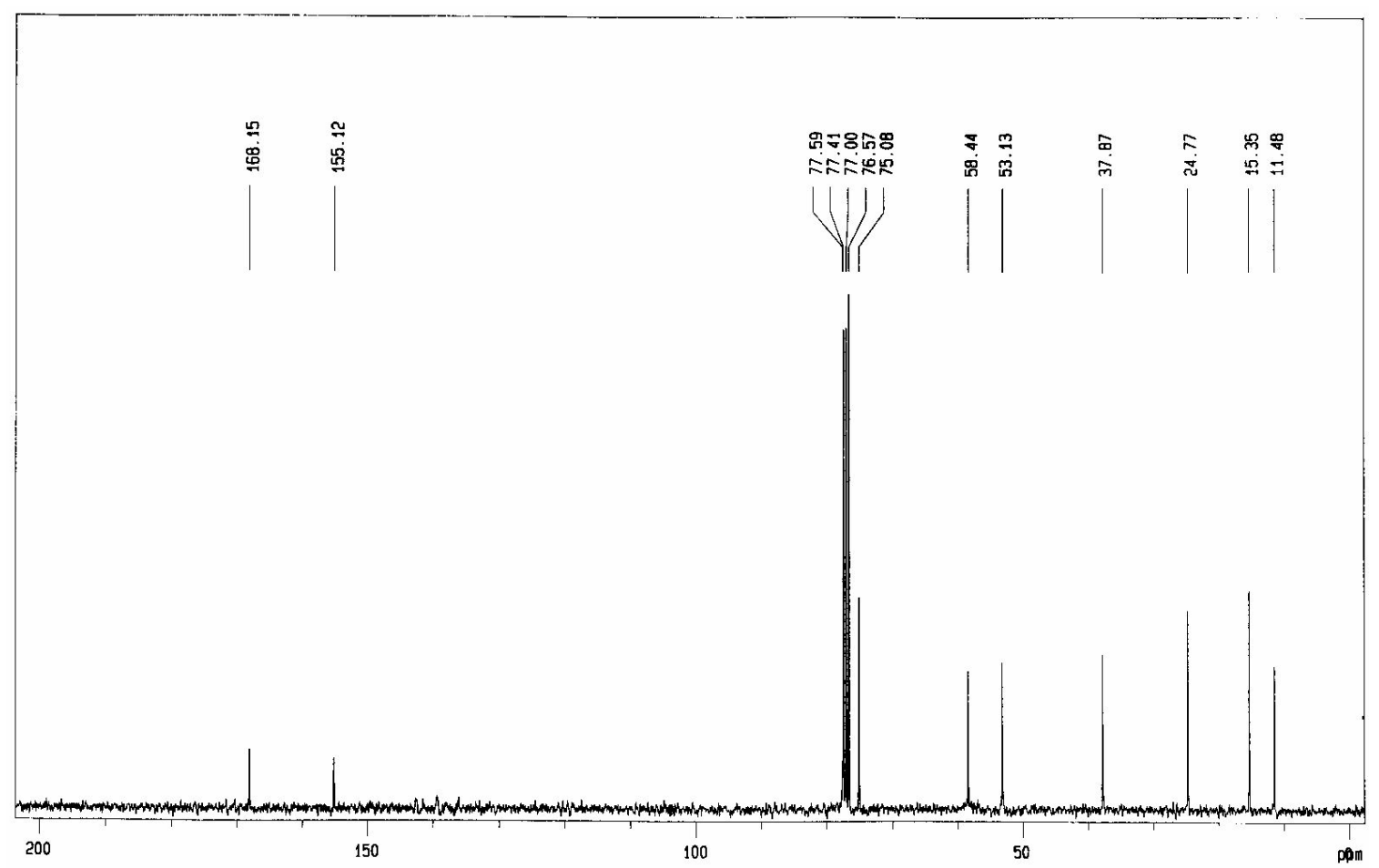




\section{Compound 6g}<smiles>C#CCOC(=O)NC(CCSC)C(=O)Oc1c(F)c(F)c(F)c(F)c1F</smiles>

${ }^{19} \mathrm{~F}$ NMR $\left(282 \mathrm{MHz}, \mathrm{CDCl}_{3}\right)$

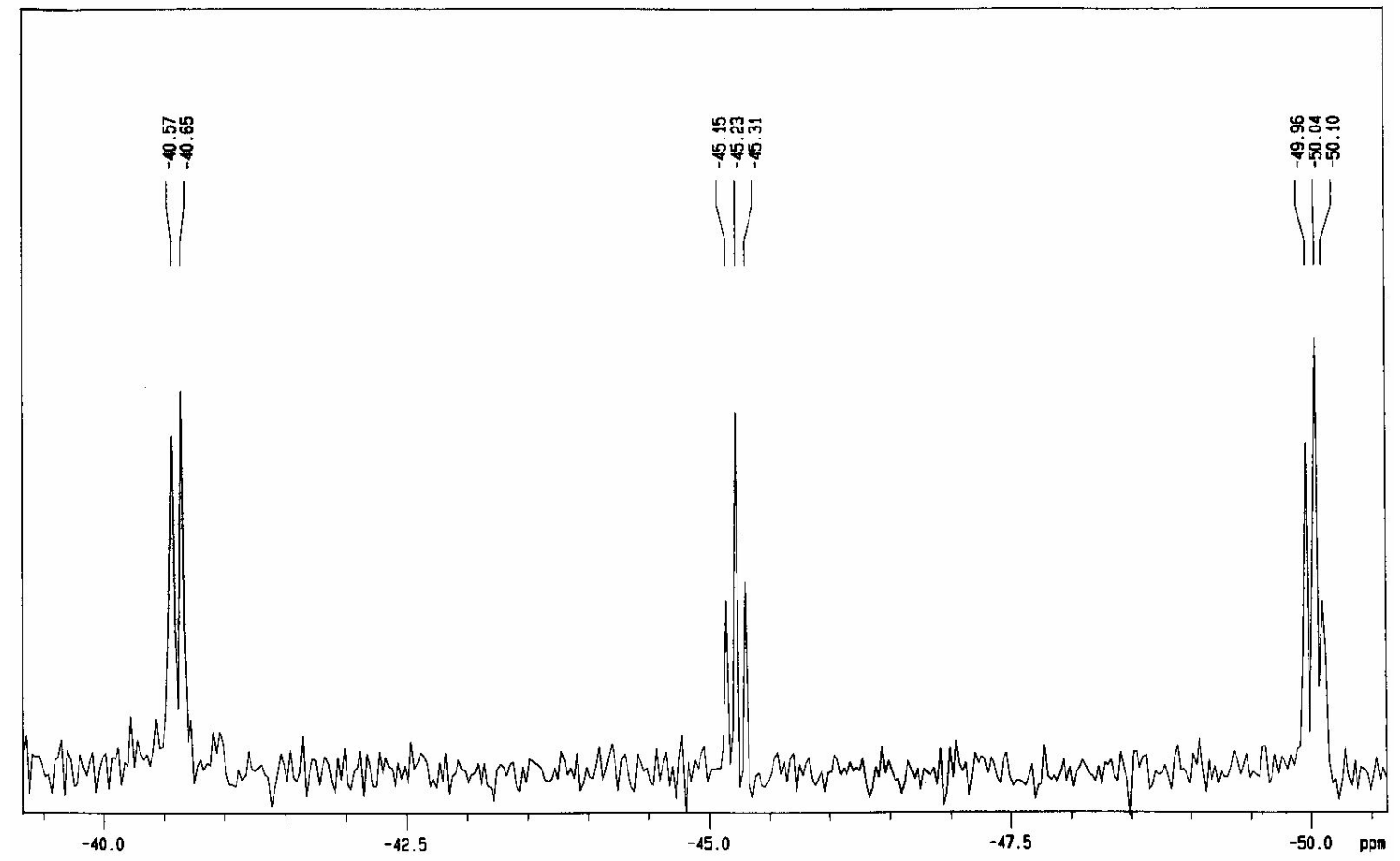




\section{Compound 6g}

${ }^{1} \mathrm{H}$ NMR (300 MHz, $\mathrm{CDCl}_{3}$ )

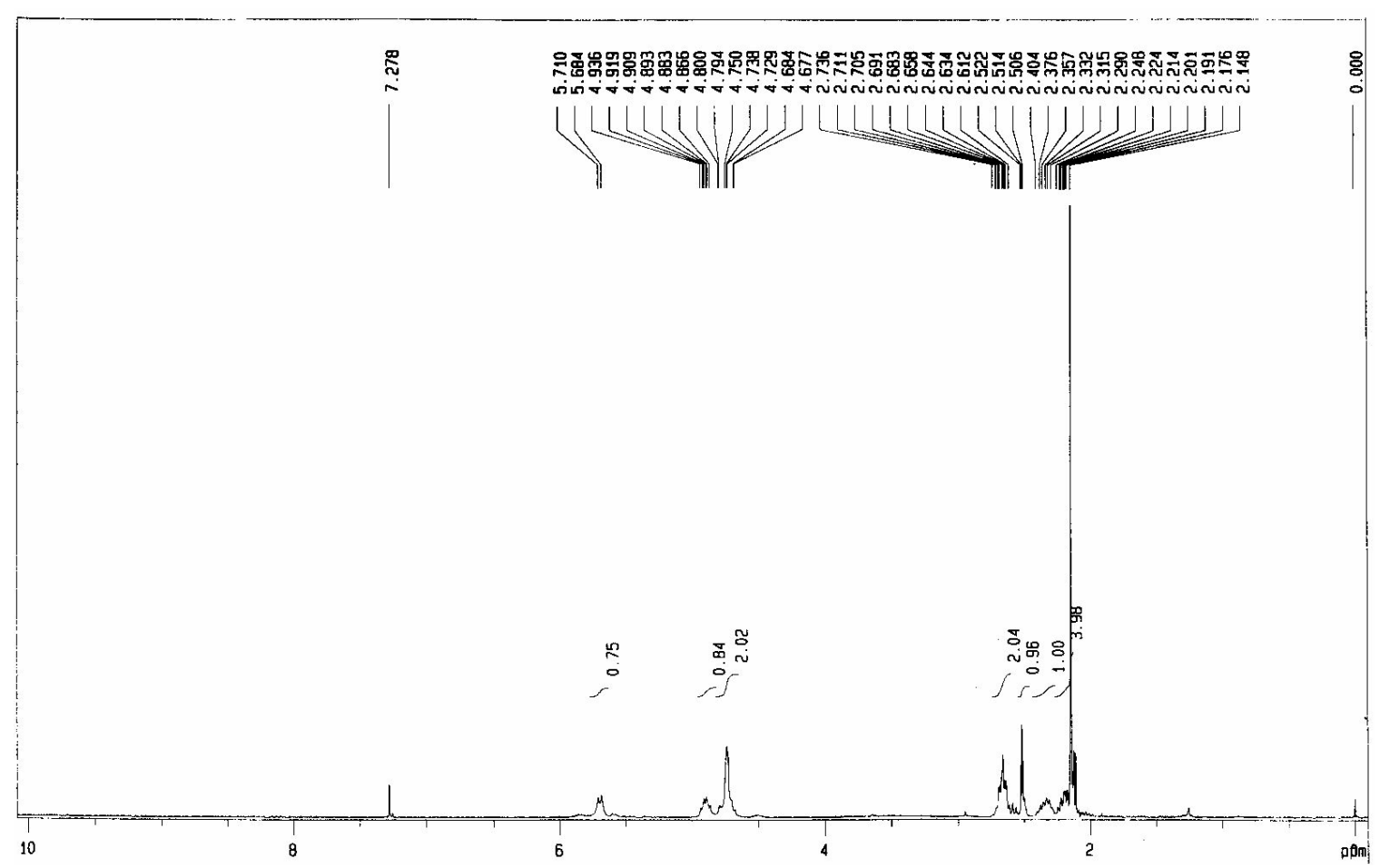

${ }^{13} \mathrm{C}$ NMR $\left(75 \mathrm{MHz}, \mathrm{CDCl}_{3}\right.$ )

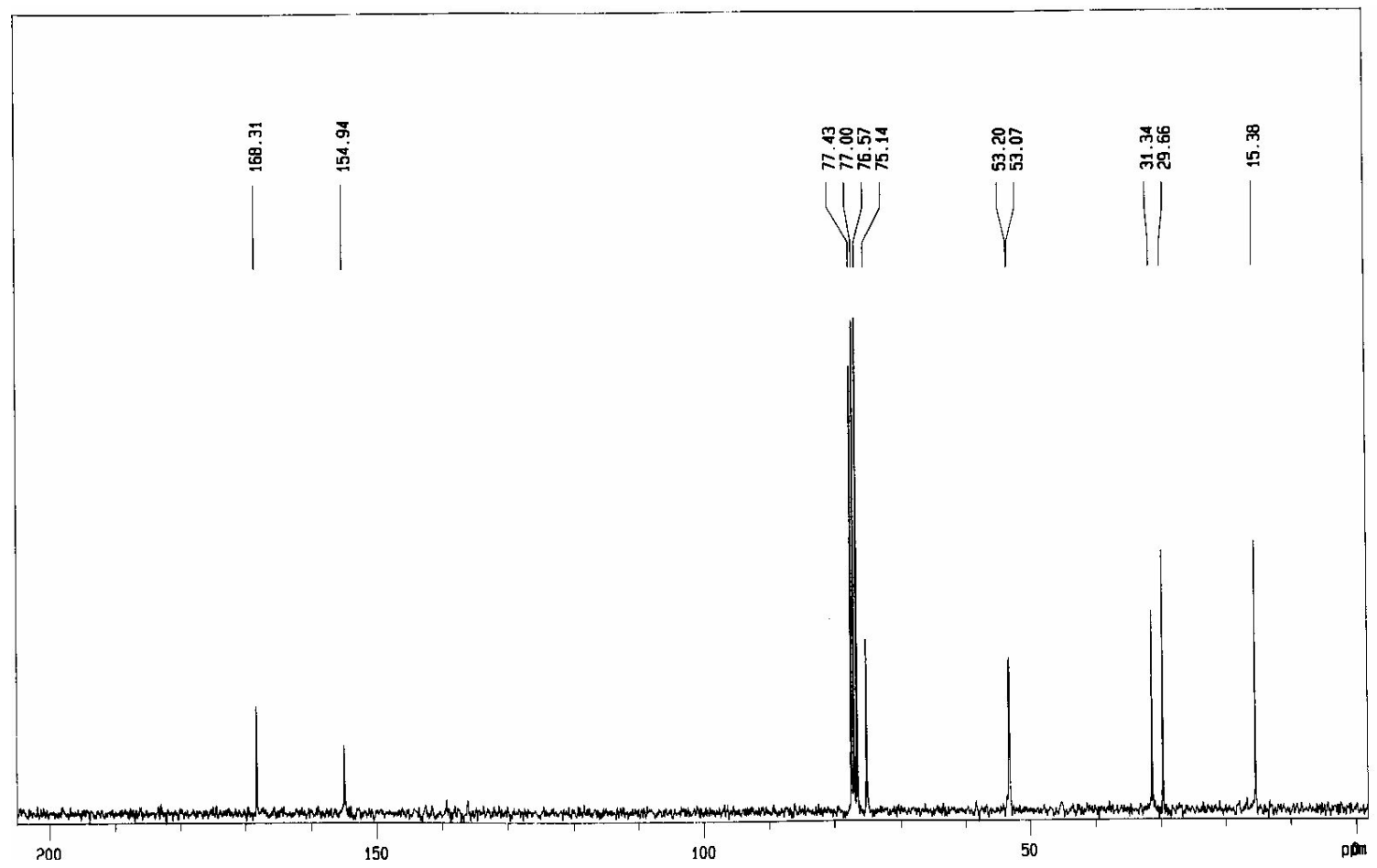




\section{Compound 6h}<smiles>C#CCOC(=O)NC(Cc1ccccc1)C(=O)Oc1c(F)c(F)c(F)c(F)c1F</smiles>

${ }^{19} \mathrm{~F}$ NMR $\left(282 \mathrm{MHz}, \mathrm{CDCl}_{3}\right)$

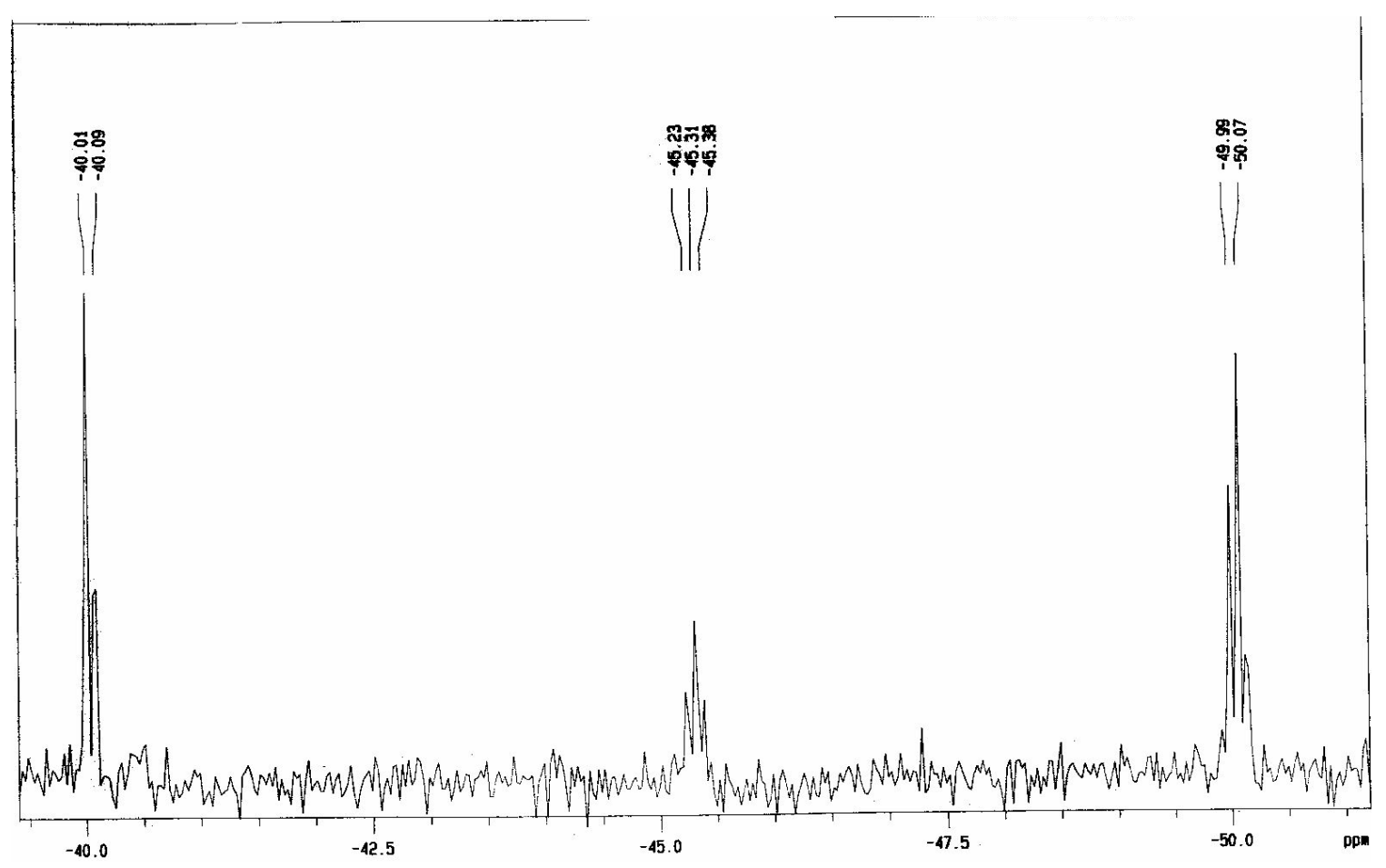




\section{Compound 6h}

${ }^{1} \mathrm{H}$ NMR (300 MHz, $\mathrm{CDCl}_{3}$ )

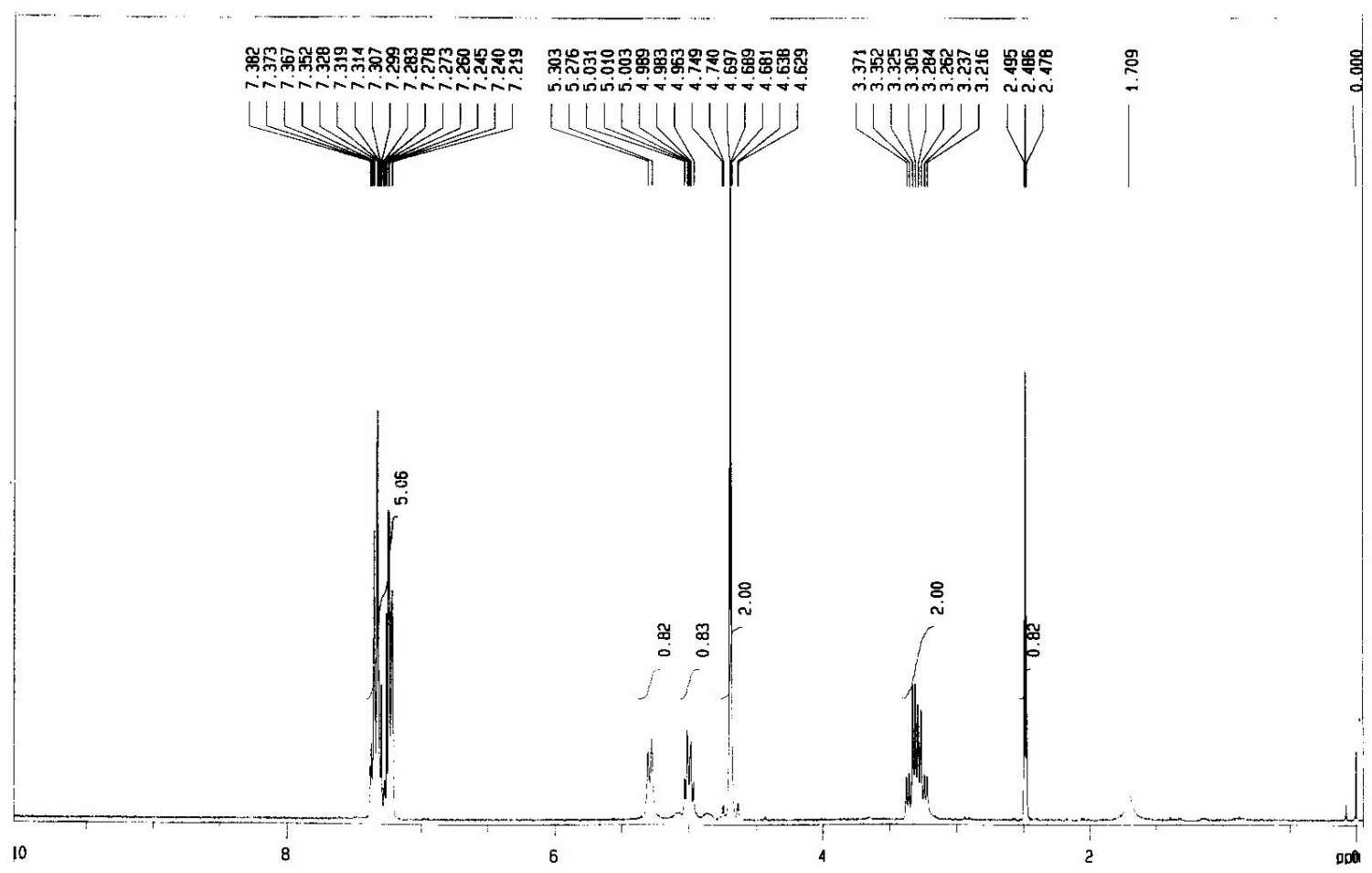

${ }^{13} \mathrm{C}$ NMR $\left(75 \mathrm{MHz}, \mathrm{CDCl}_{3}\right)$

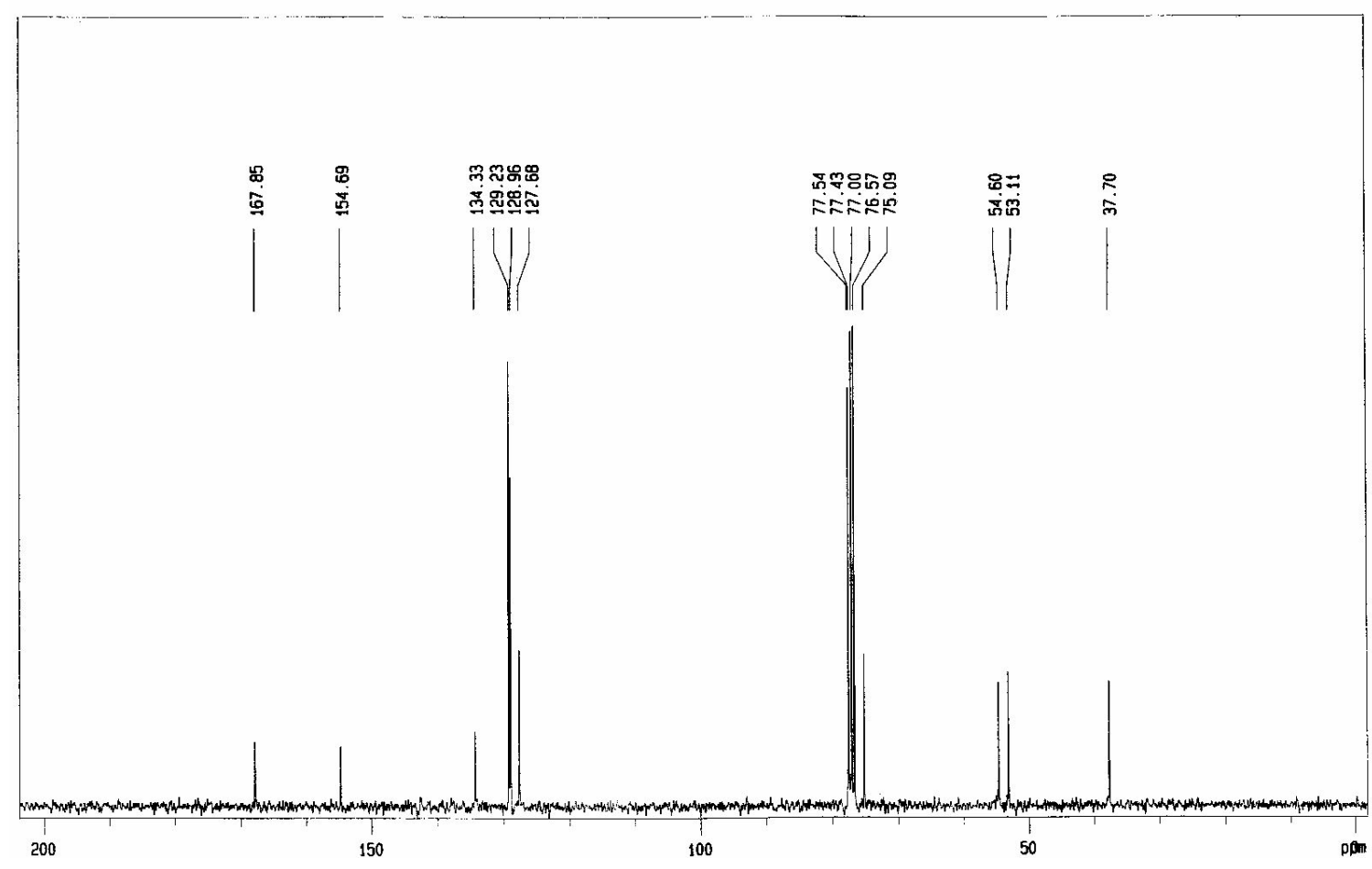




\section{Compound 6i}

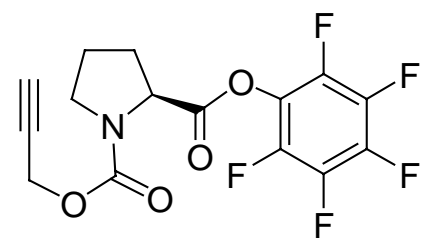

${ }^{19} \mathrm{~F}$ NMR $\left(282 \mathrm{MHz}, \mathrm{CDCl}_{3}\right)$

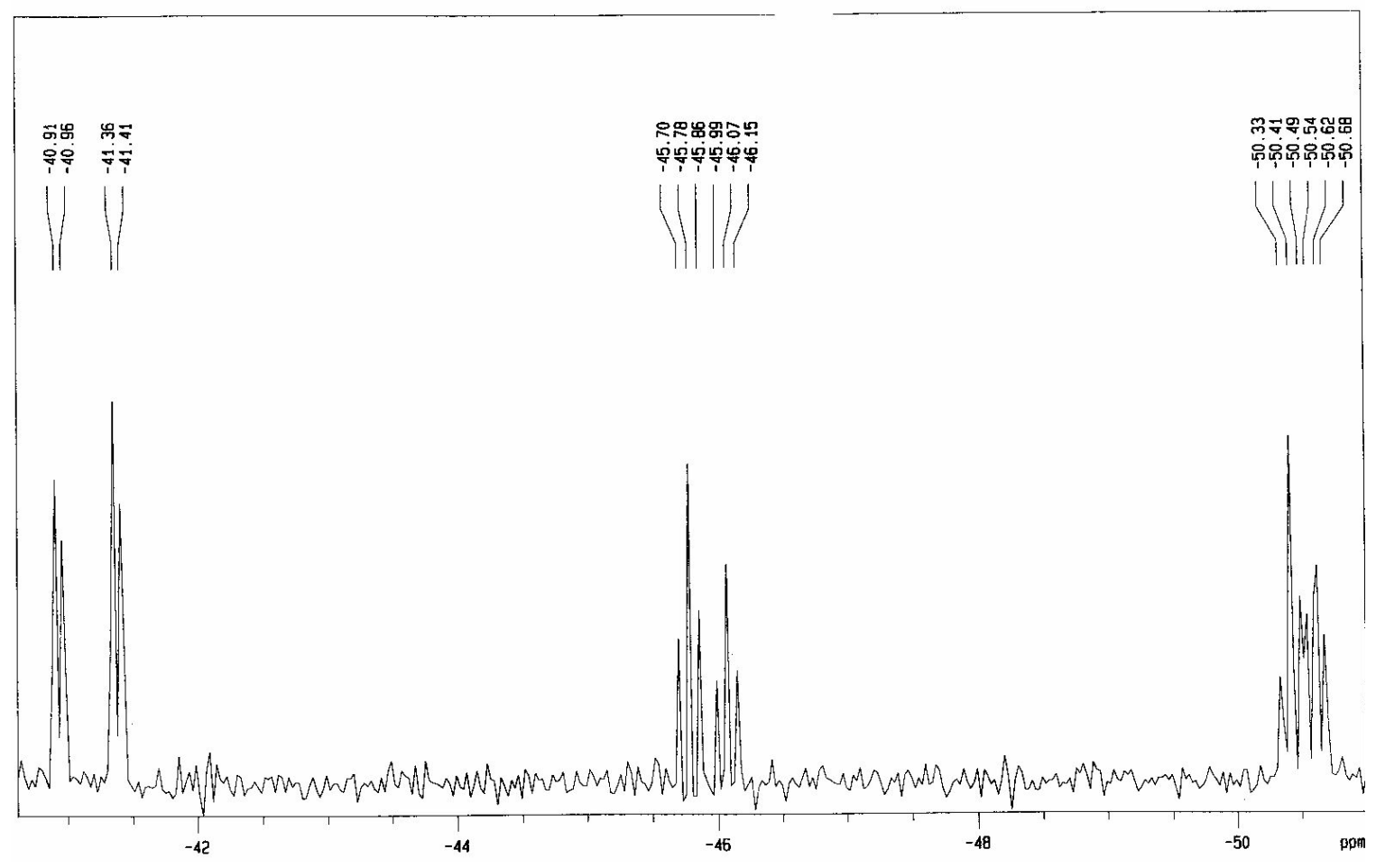




\section{Compound 6i}

${ }^{1} \mathrm{H}$ NMR $\left(300 \mathrm{MHz}, \mathrm{CDCl}_{3}\right.$ )

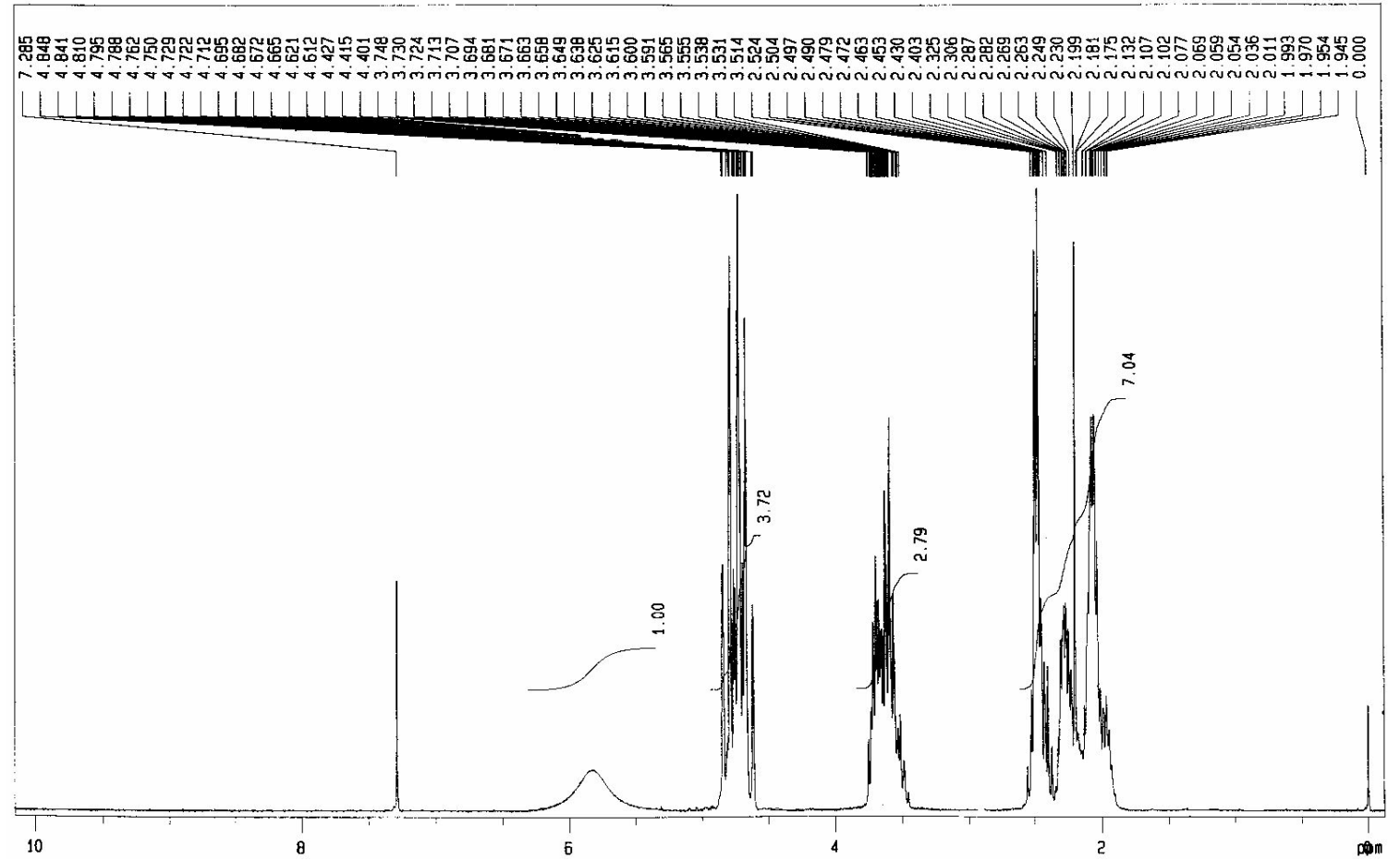

${ }^{13} \mathrm{C}$ NMR $\left(75 \mathrm{MHz}, \mathrm{CDCl}_{3}\right.$ )

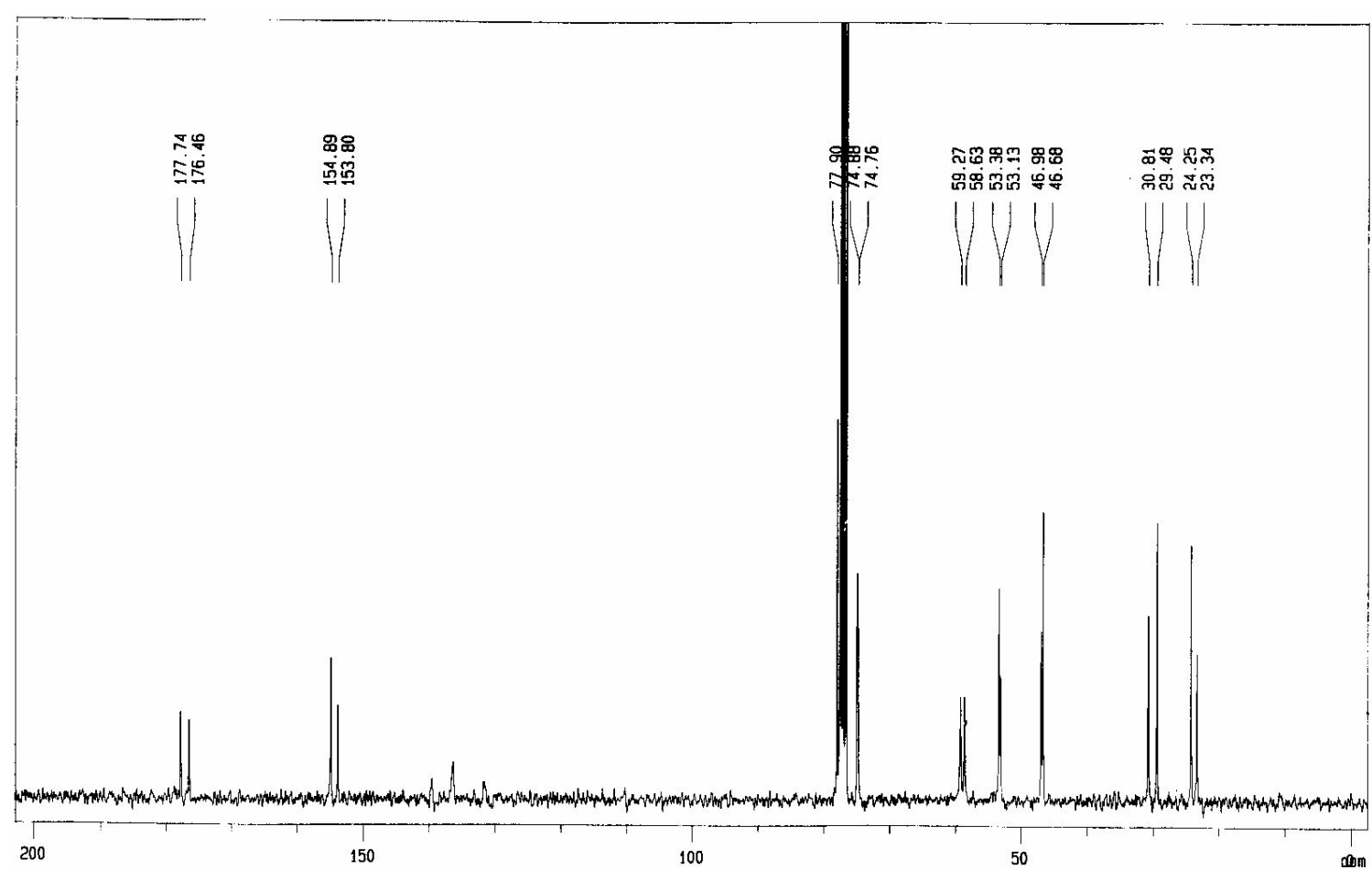




\section{Compound 6j}<smiles>C#CCOC(=O)NC(C)(C)C(=O)Oc1c(F)c(F)c(F)c(F)c1F</smiles>

${ }^{19} \mathrm{~F}$ NMR $\left(282 \mathrm{MHz}, \mathrm{CDCl}_{3}\right)$

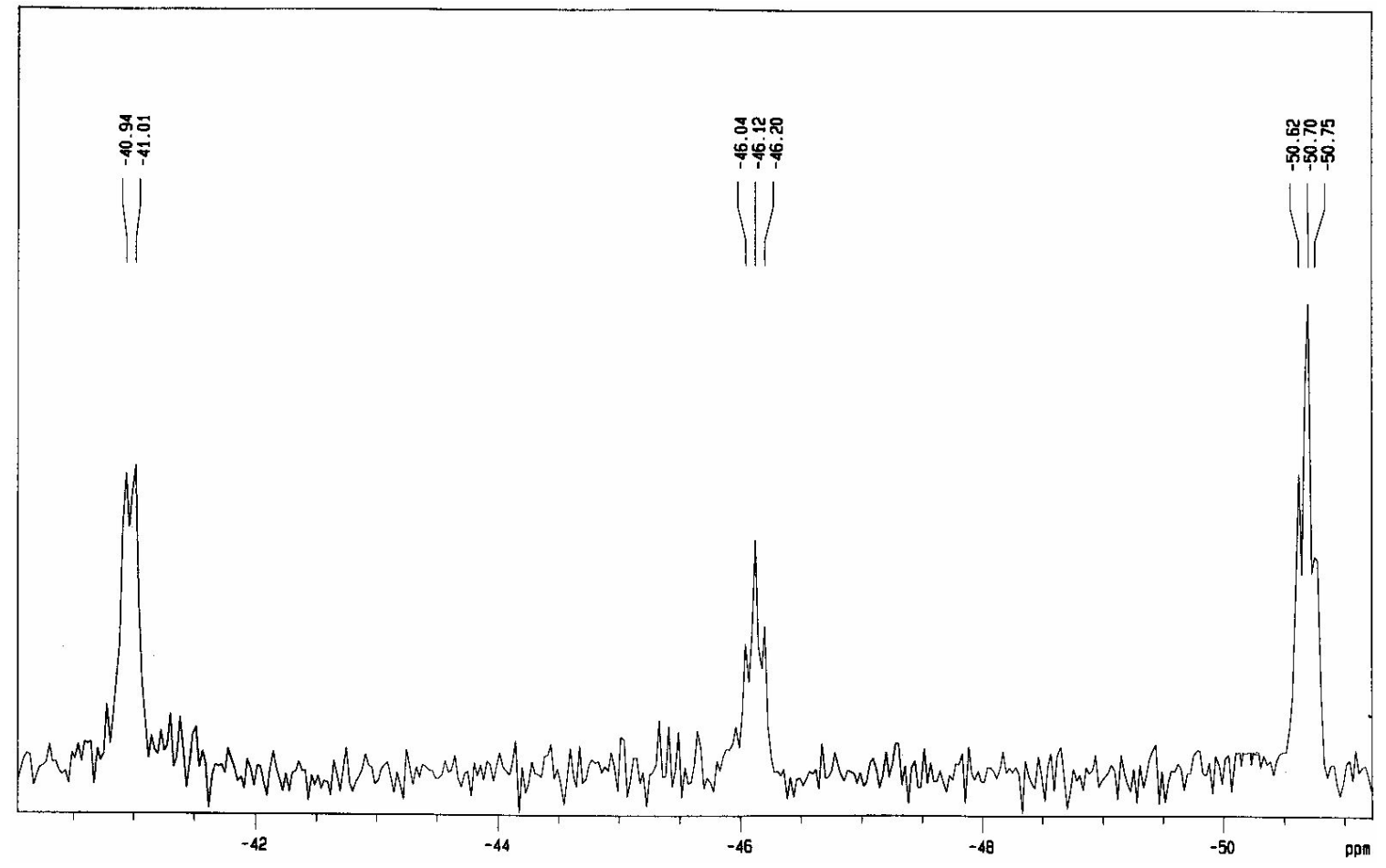




\section{Compound 6j}

${ }^{1} \mathrm{H}$ NMR $\left(300 \mathrm{MHz}, \mathrm{CDCl}_{3}\right.$ )

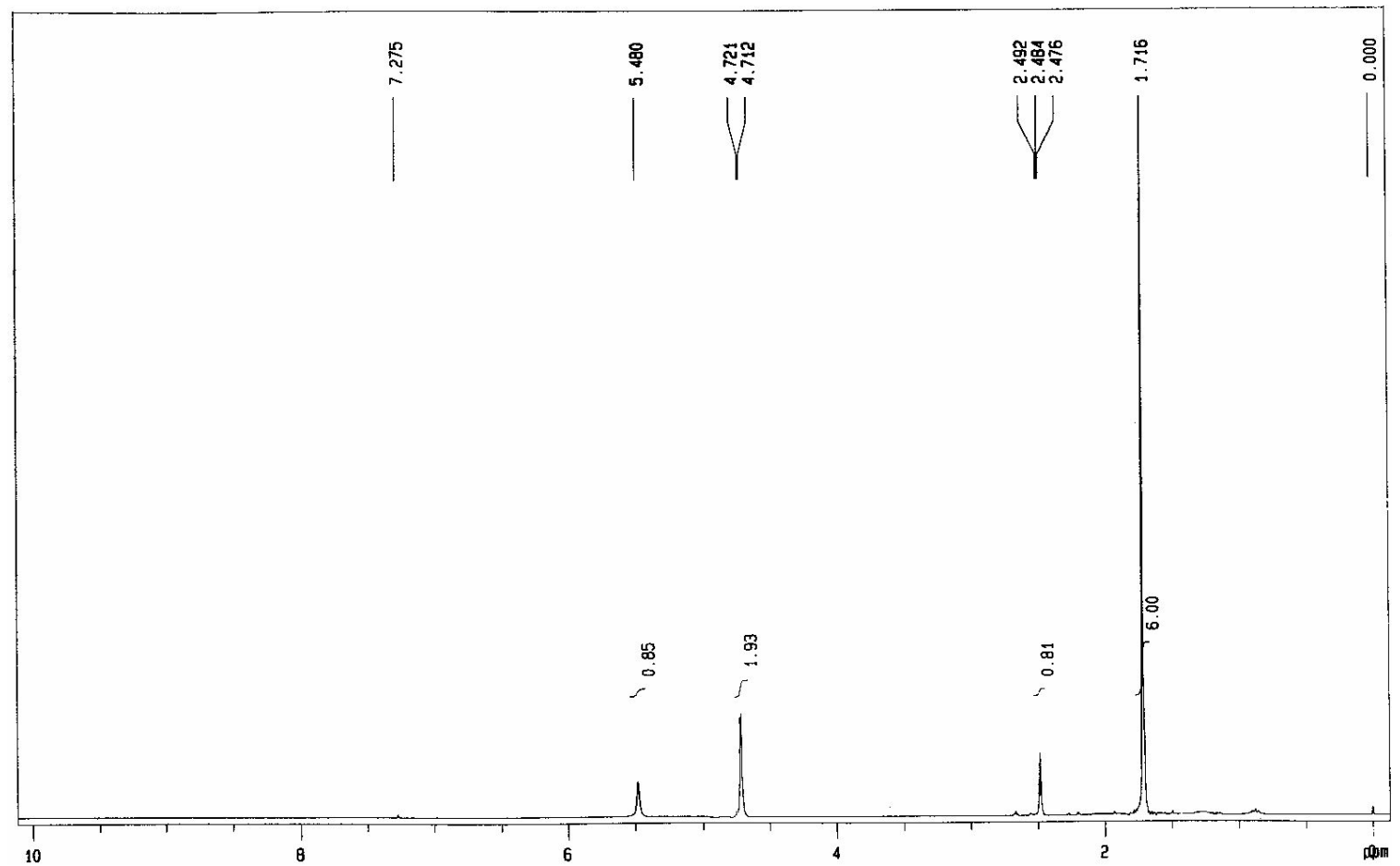

${ }^{13} \mathrm{C}$ NMR $\left(75 \mathrm{~Hz}, \mathrm{CDCl}_{3}\right)$

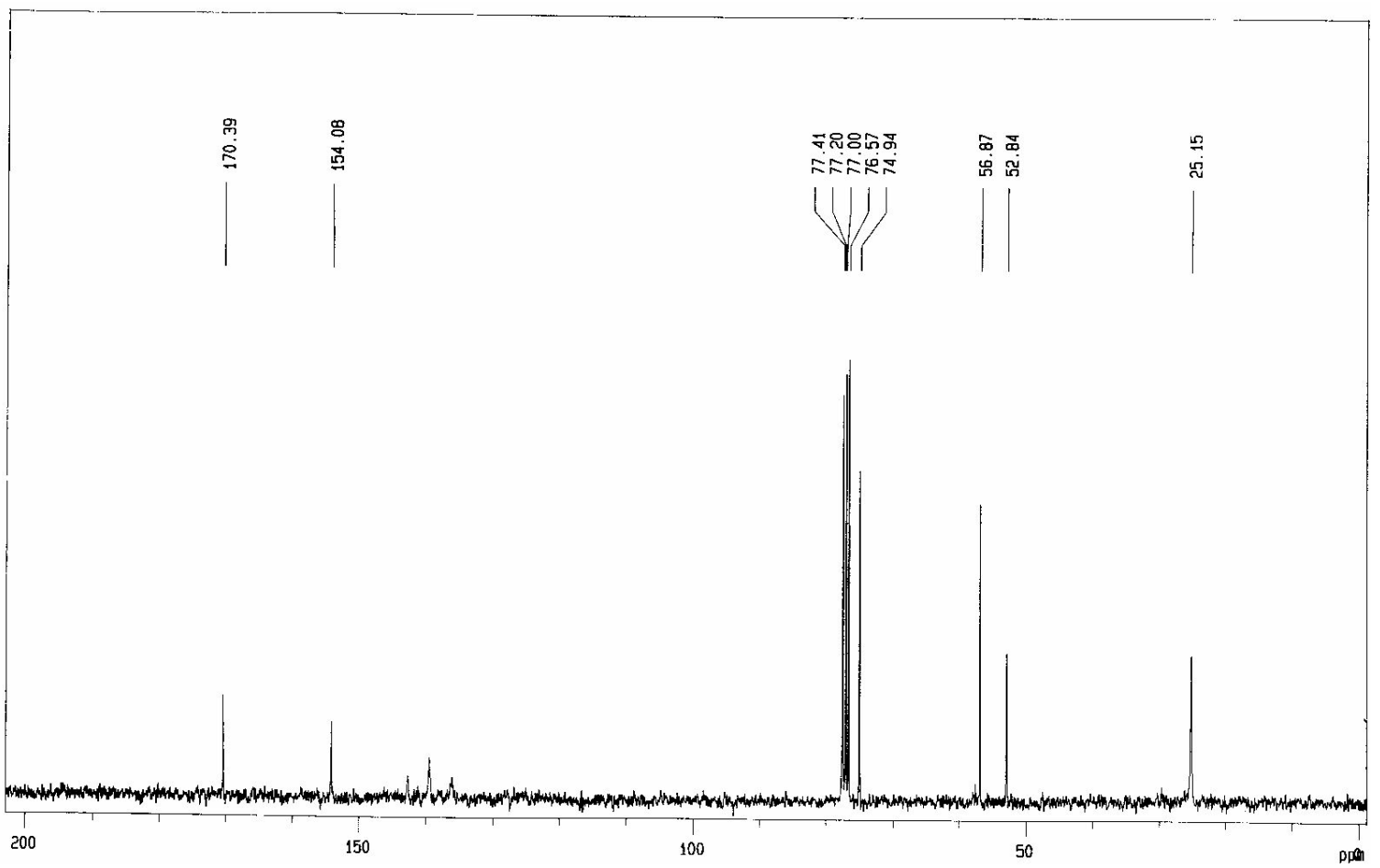




\section{Compound 6k}<smiles>C#CCOC(=O)NCCC(=O)Oc1c(F)c(F)c(F)c(F)c1F</smiles>

$\left.{ }^{19} \mathrm{~F} \mathrm{NMR} \mathrm{(282} \mathrm{MHz,} \mathrm{CDCl}_{3}\right)$

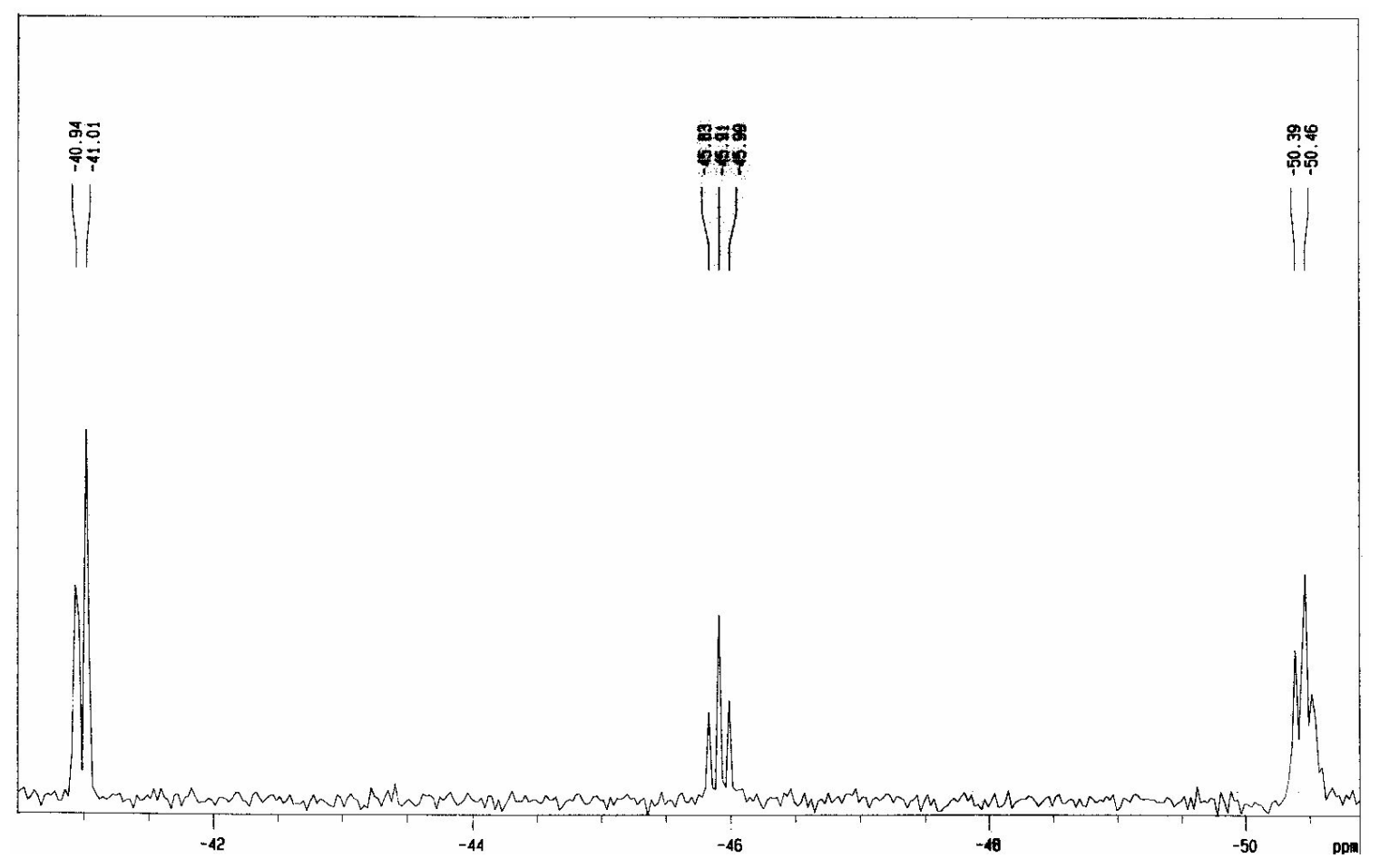




\section{Compound 6k}

${ }^{1} \mathrm{H}$ NMR $\left(300 \mathrm{MHz}, \mathrm{CDCl}_{3}\right.$ )

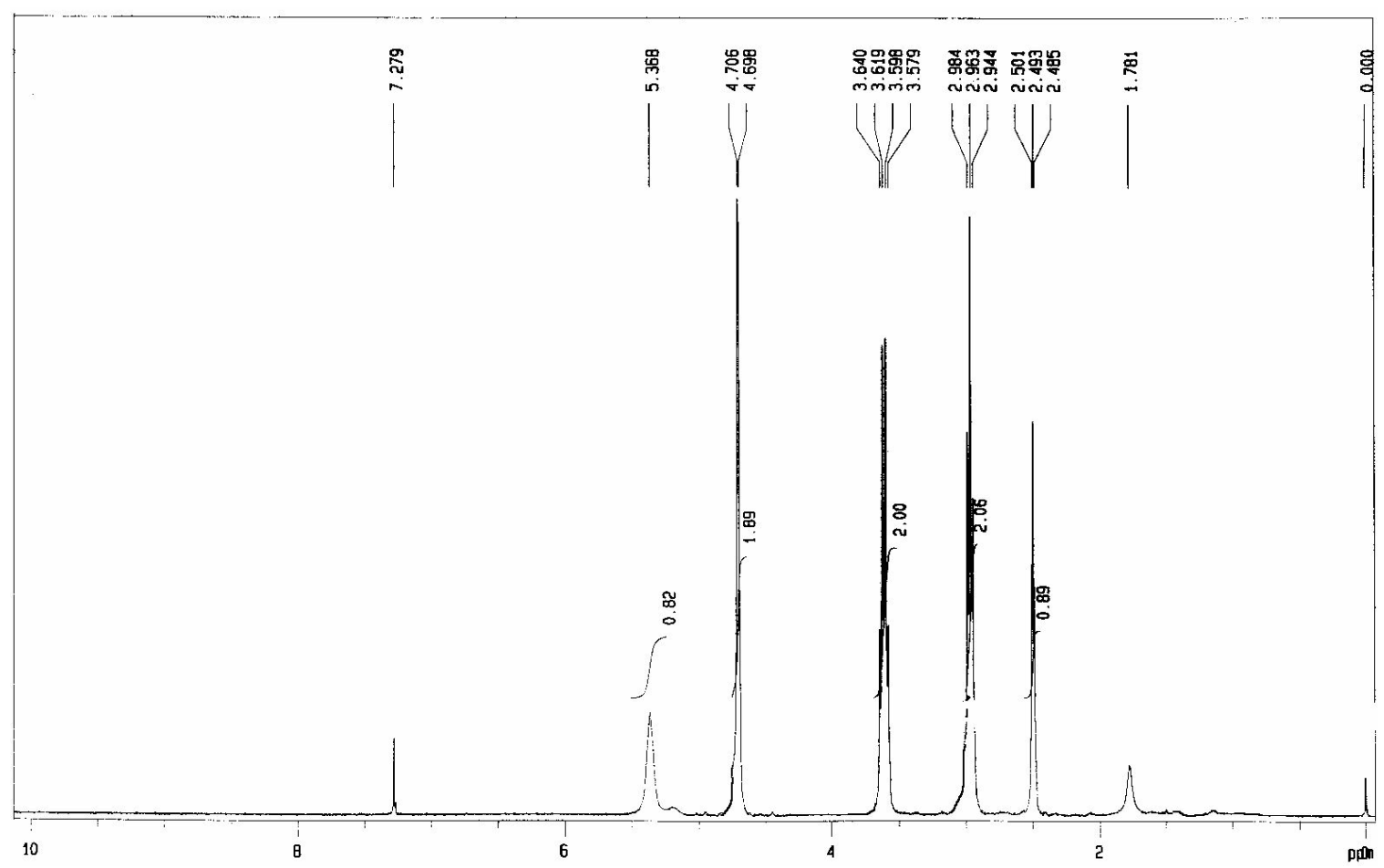

${ }^{13} \mathrm{C}$ NMR $\left(75 \mathrm{MHz}, \mathrm{CDCl}_{3}\right)$
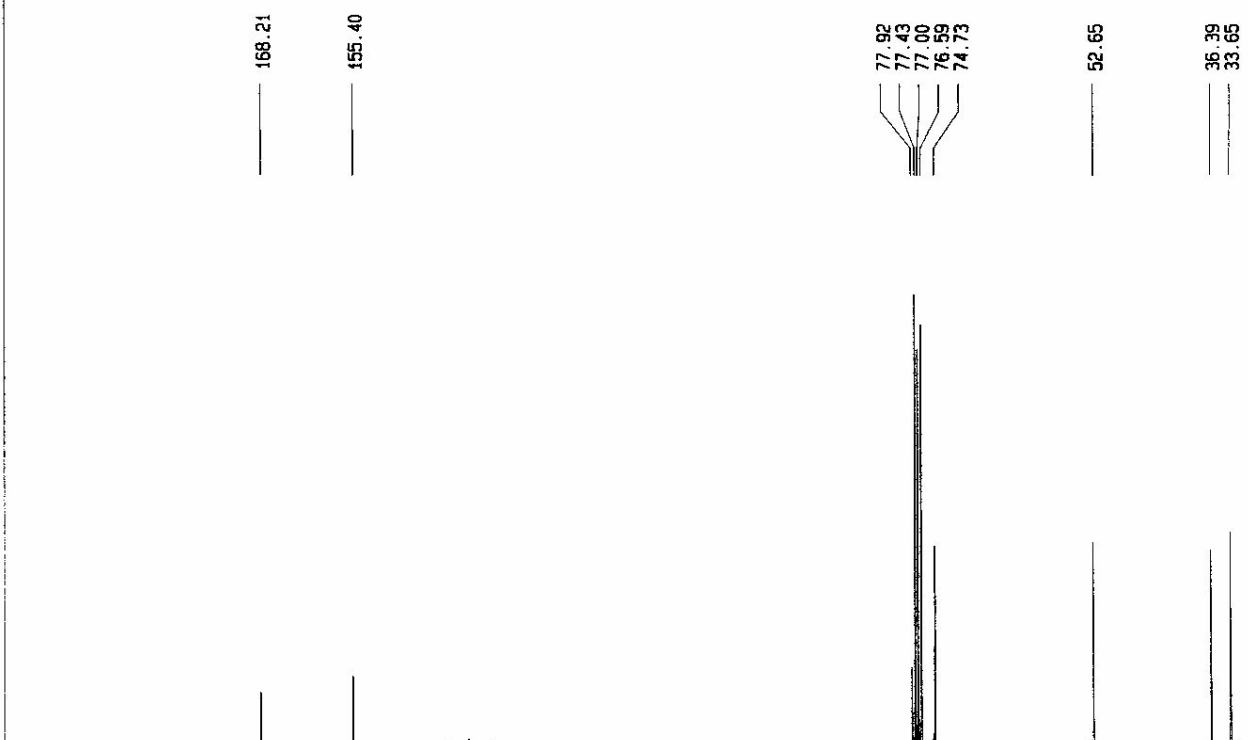


\section{Compound 61}<smiles>C#CCOC(=O)NCCCC(=O)Oc1c(F)c(F)c(F)c(F)c1F</smiles>

${ }^{19} \mathrm{~F}$ NMR (282 MHz, $\mathrm{CDCl}_{3}$ )

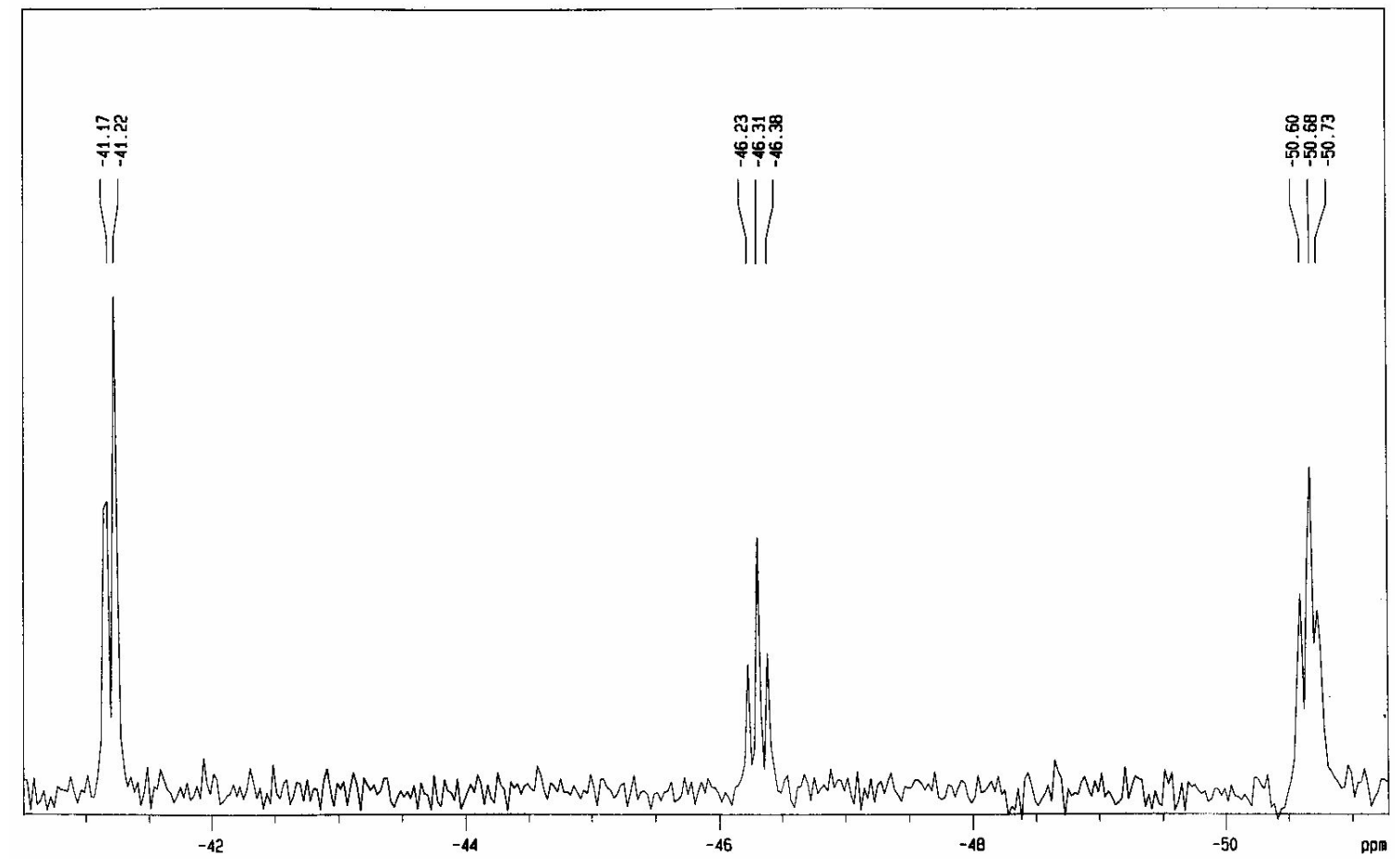




\section{Compound 61}

${ }^{1} \mathrm{H}$ NMR $\left(300 \mathrm{MHz}, \mathrm{CDCl}_{3}\right.$ )

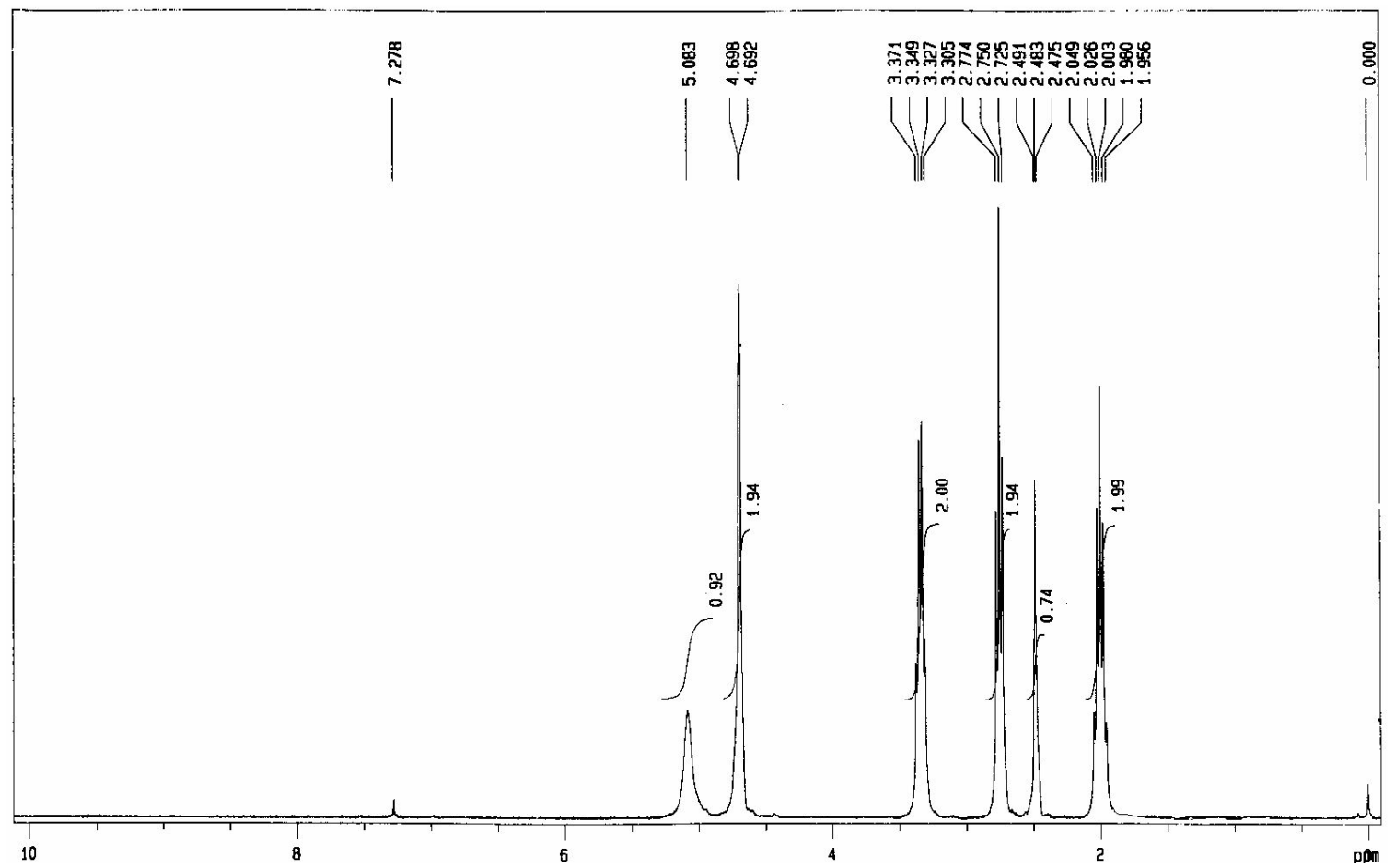

${ }^{13} \mathrm{C}$ NMR $\left(75 \mathrm{MHz}, \mathrm{CDCl}_{3}\right)$

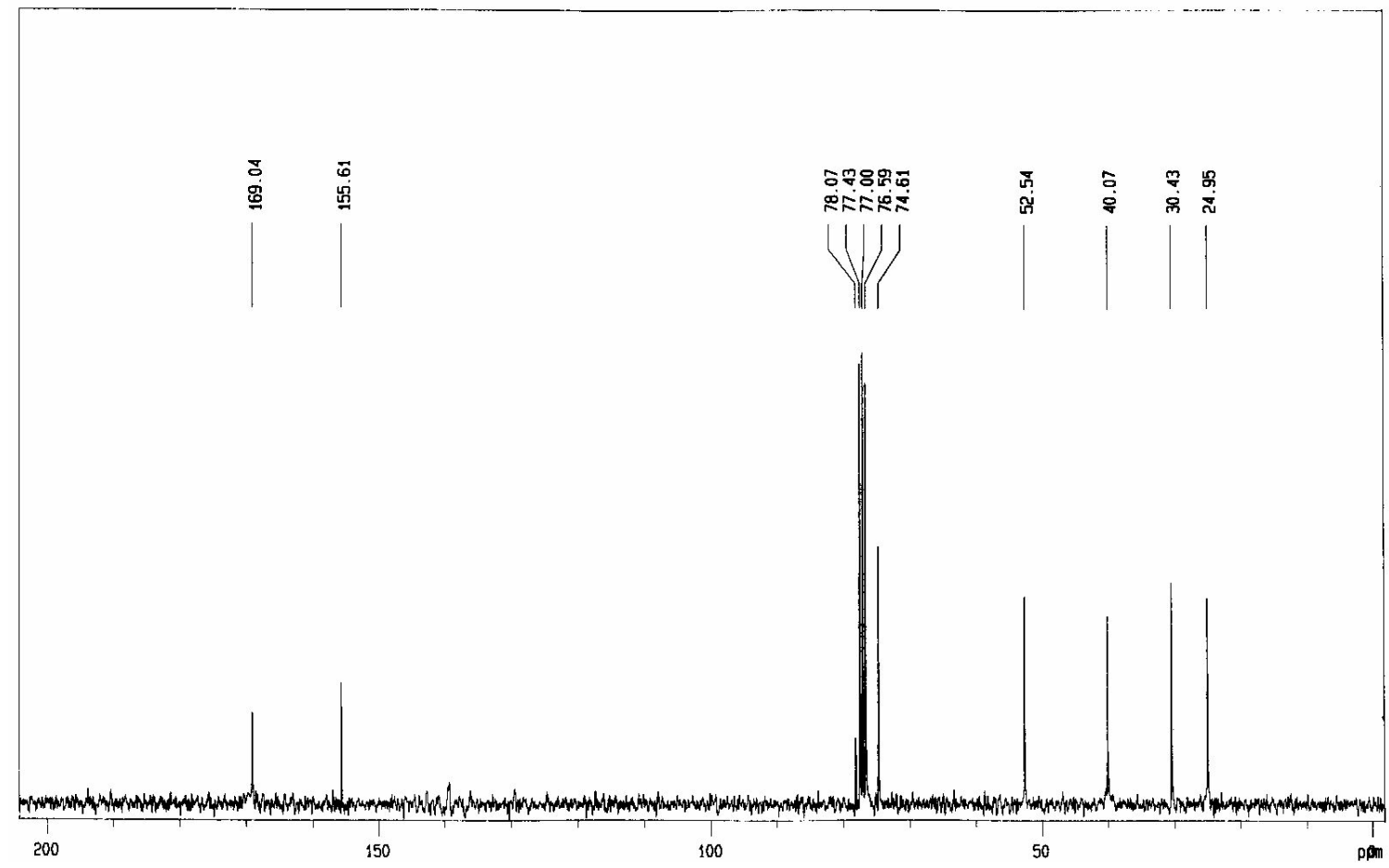




\section{Compound 6m}<smiles>C#CCOC(=O)NC(C(=O)Oc1c(F)c(F)c(F)c(F)c1F)c1ccccc1</smiles>

${ }^{19}$ F NMR $\left(282 \mathrm{MHz}, \mathrm{CDCl}_{3}\right)$

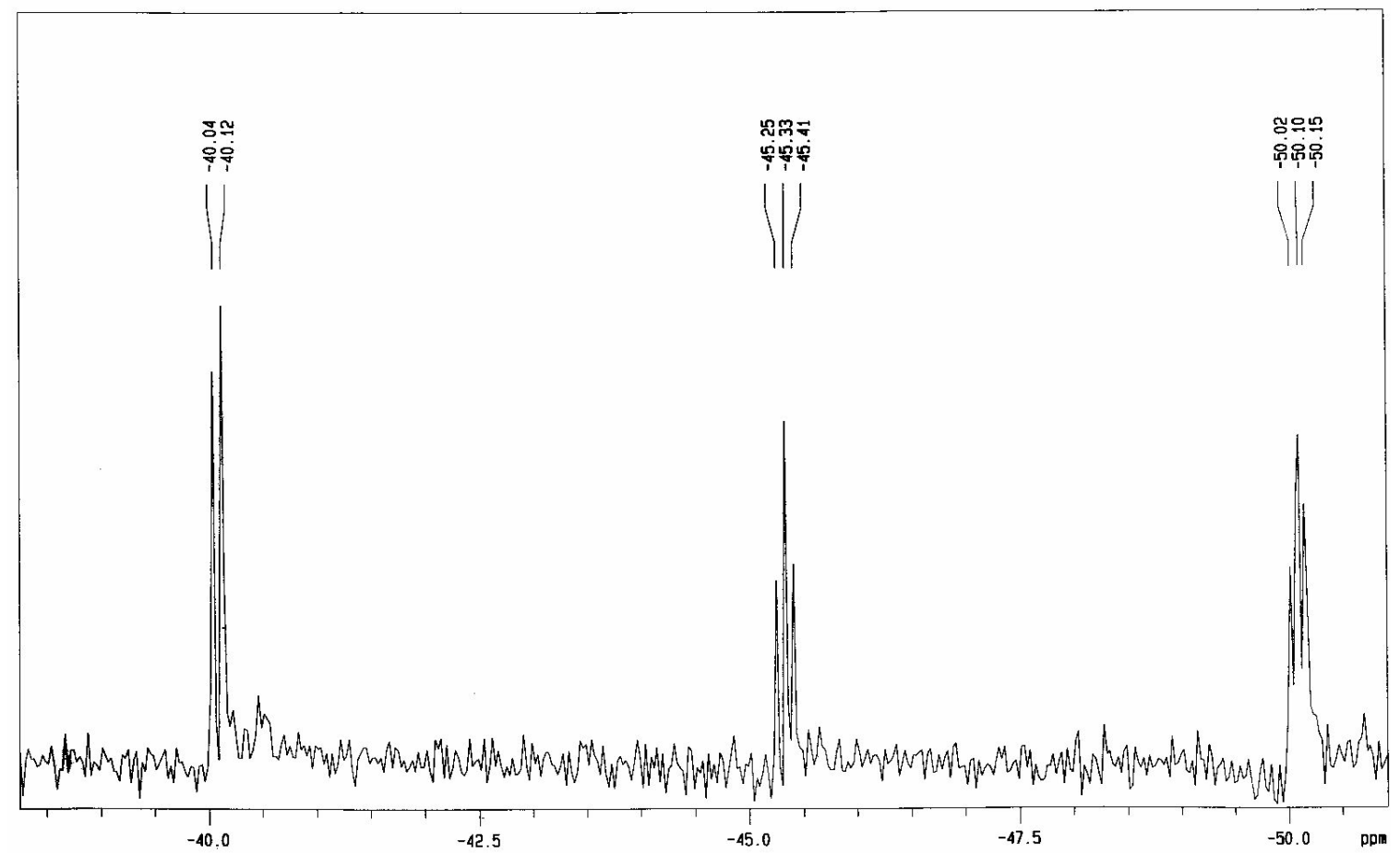




\section{Compound 6m}

${ }^{1} \mathrm{H}$ NMR $\left(300 \mathrm{MHz}, \mathrm{CDCl}_{3}\right.$ )

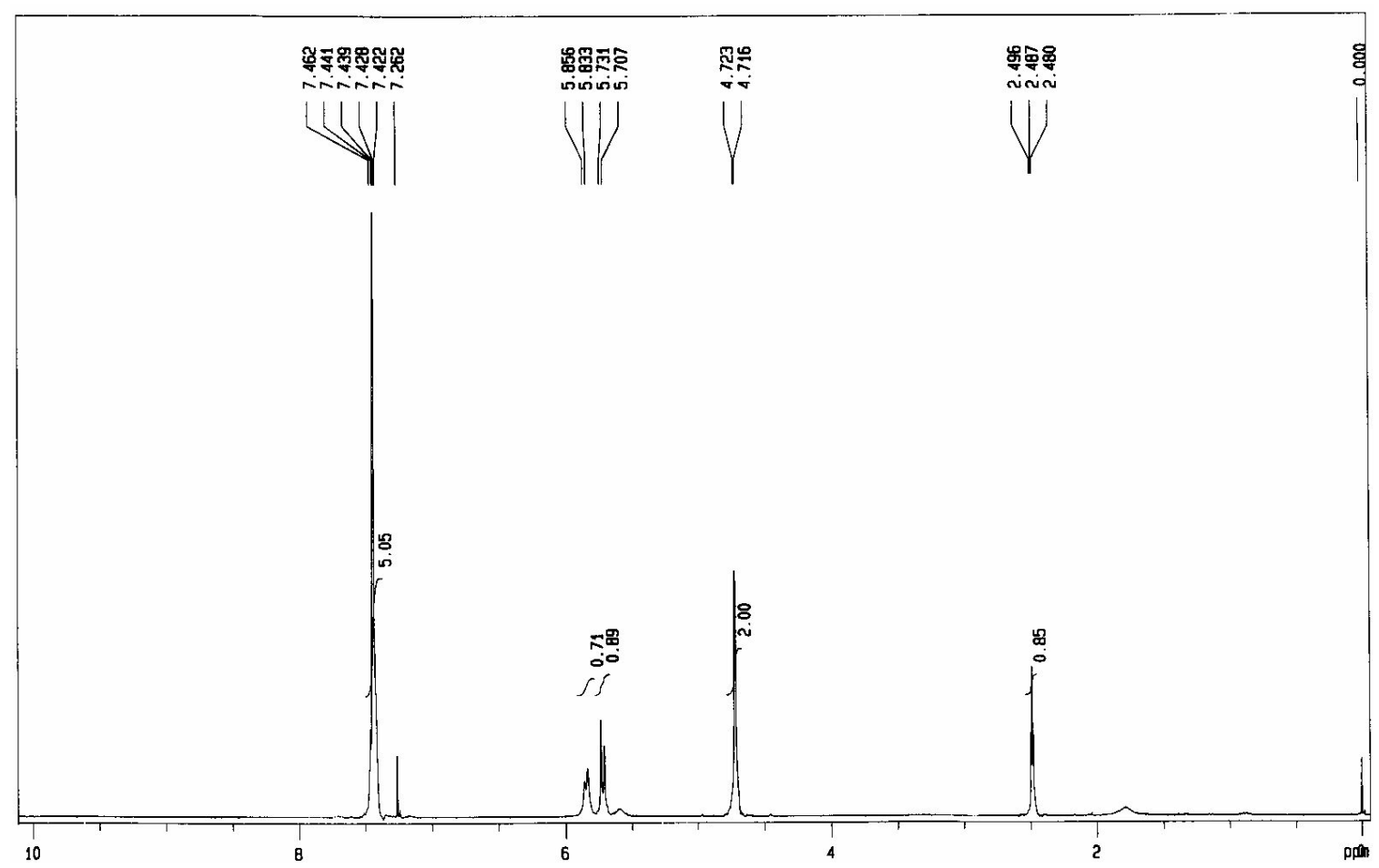

${ }^{13} \mathrm{C}$ NMR $\left(75 \mathrm{MHz}, \mathrm{CDCl}_{3}\right.$ )

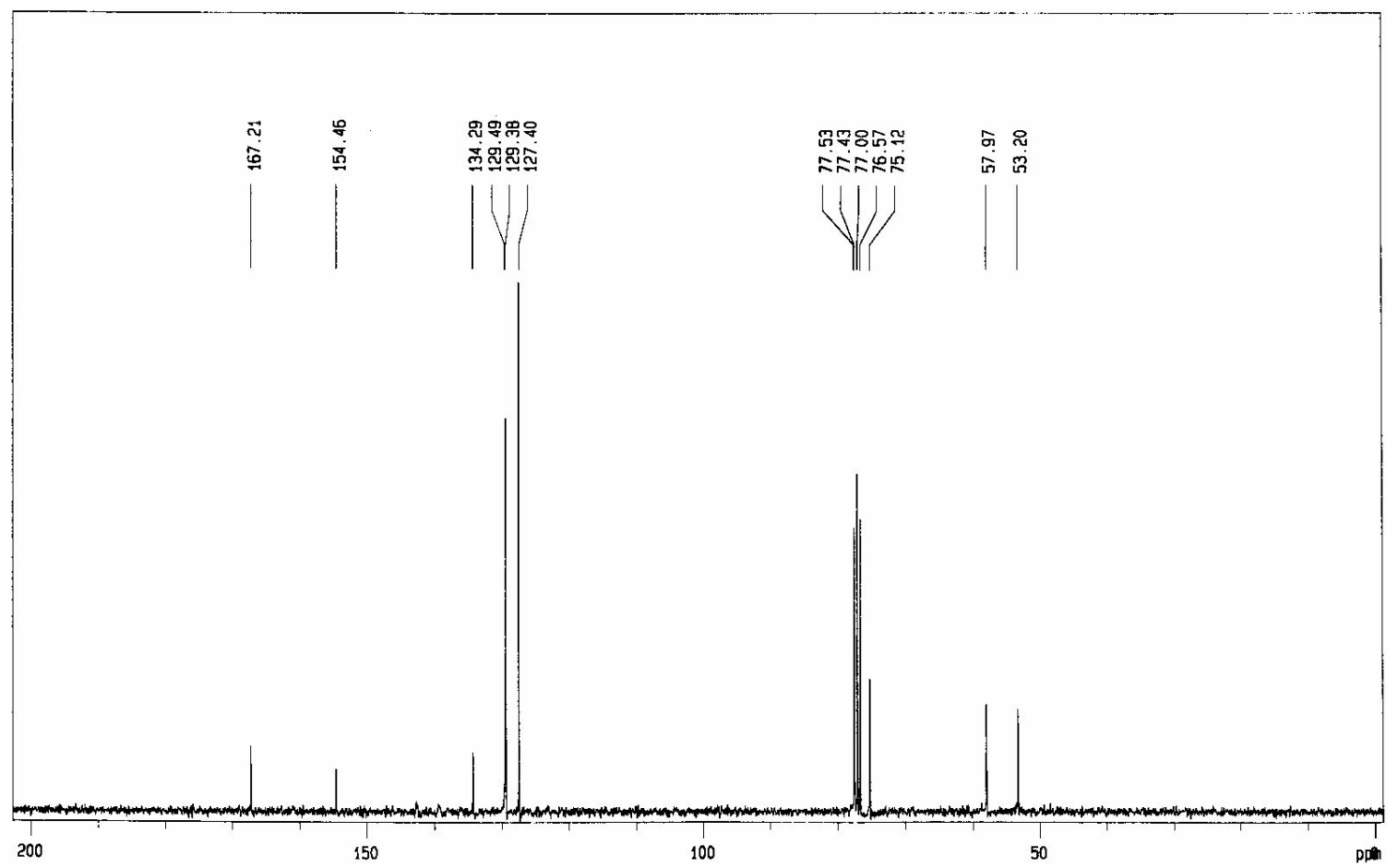




\section{Compound 6n}<smiles>C#CCOC(=O)NCCCC[C@H](NC(=O)OCc1ccccc1)C(=O)Oc1c(F)c(F)c(F)c(F)c1F</smiles>

${ }^{19} \mathrm{~F}$ NMR $\left(282 \mathrm{MHz}, \mathrm{CDCl}_{3}\right)$

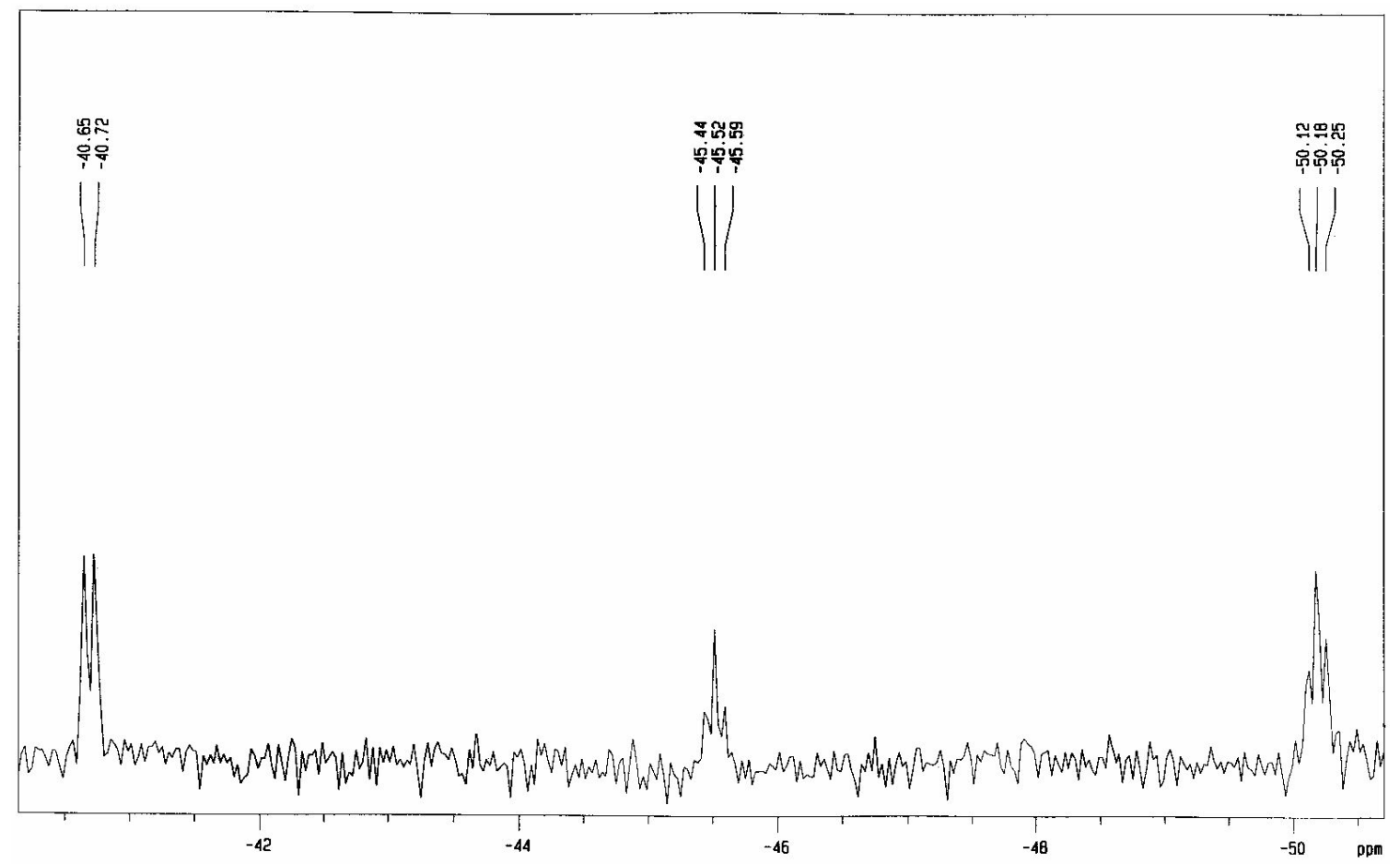




\section{Compound 6n}

${ }^{1} \mathrm{H}$ NMR $\left(300 \mathrm{MHz}, \mathrm{CDCl}_{3}\right.$ )

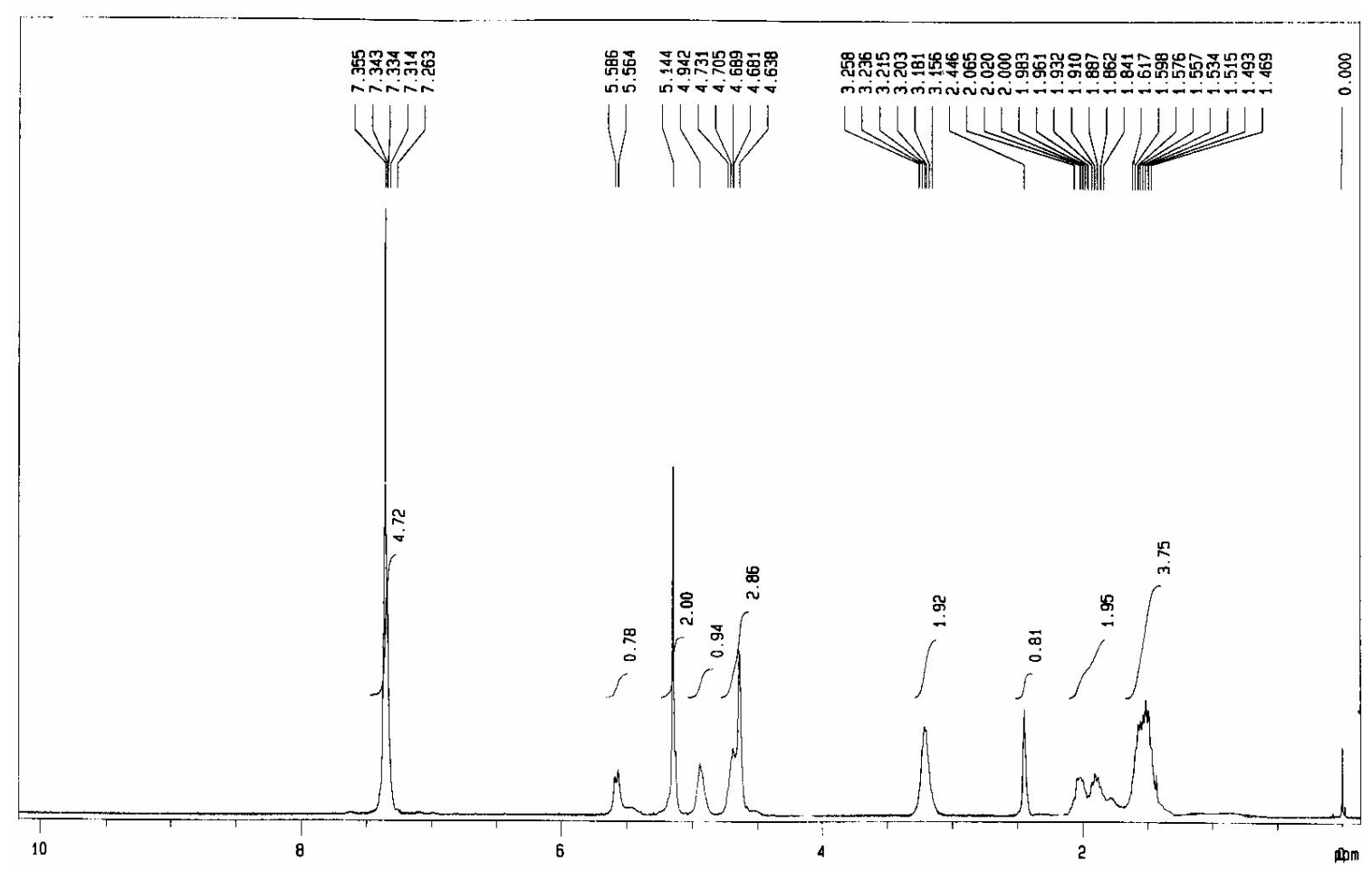

${ }^{13} \mathrm{C}$ NMR $\left(75 \mathrm{MHz}, \mathrm{CDCl}_{3}\right)$

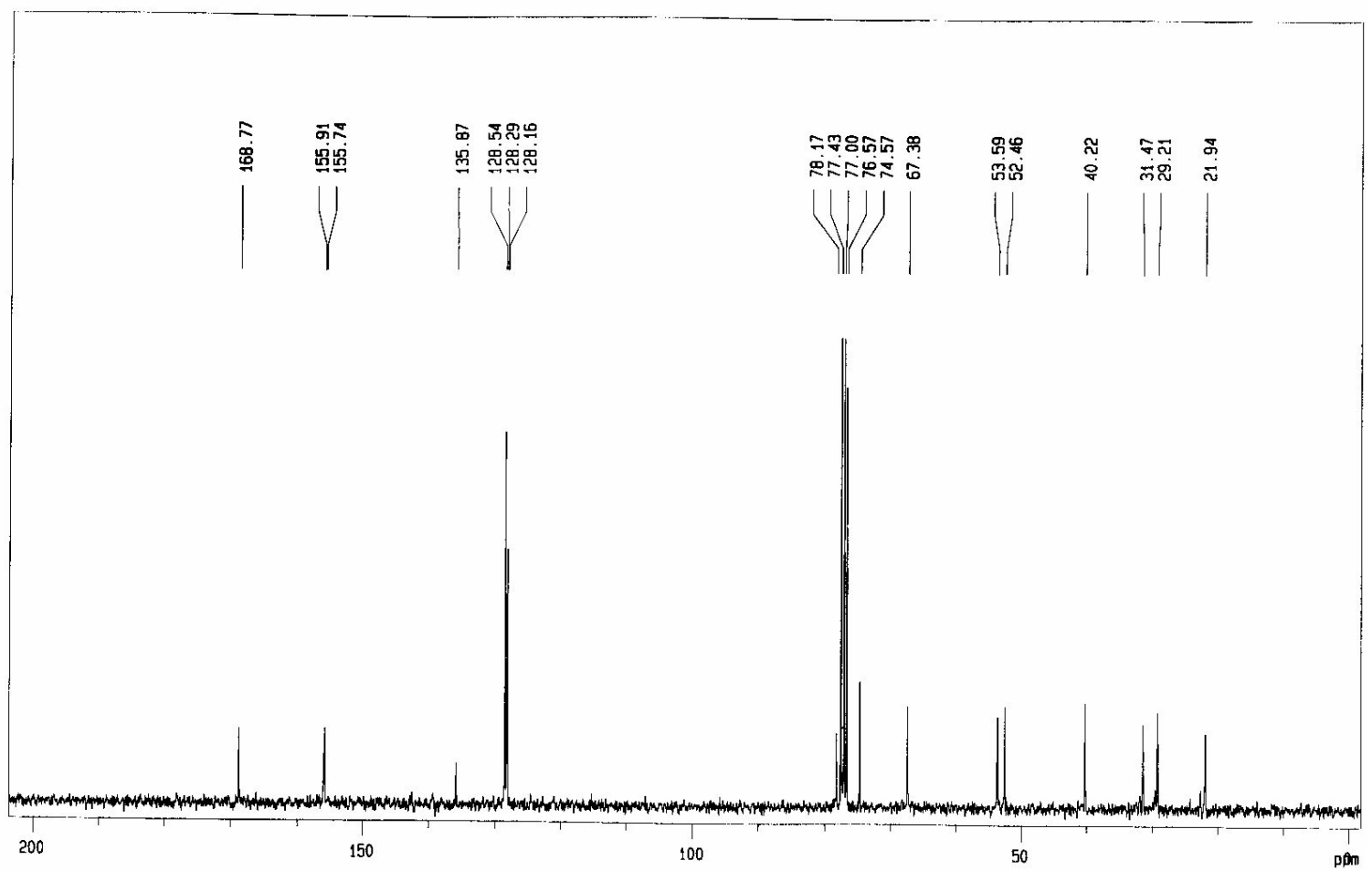




\section{Compound 6o}<smiles>C#CCOC(=O)NCCCCC(NC(=O)OCC#C)C(=O)Oc1c(F)c(F)c(F)c(F)c1F</smiles>

${ }^{19} \mathrm{~F}$ NMR $\left(282 \mathrm{MHz}, \mathrm{CDCl}_{3}\right)$

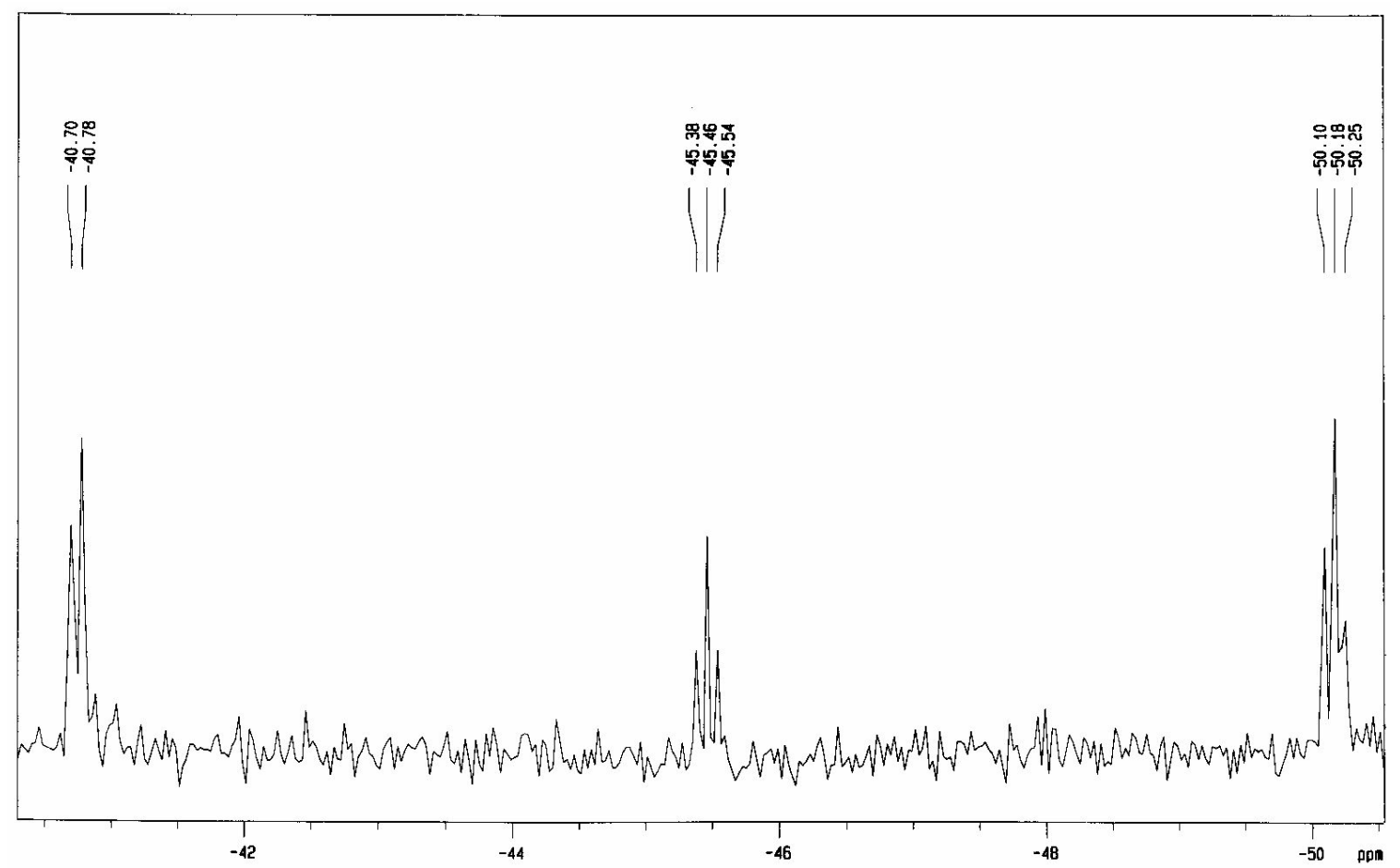




\section{Compound 6o}

${ }^{1} \mathrm{H}$ NMR $\left(300 \mathrm{MHz}, \mathrm{CDCl}_{3}\right.$ )
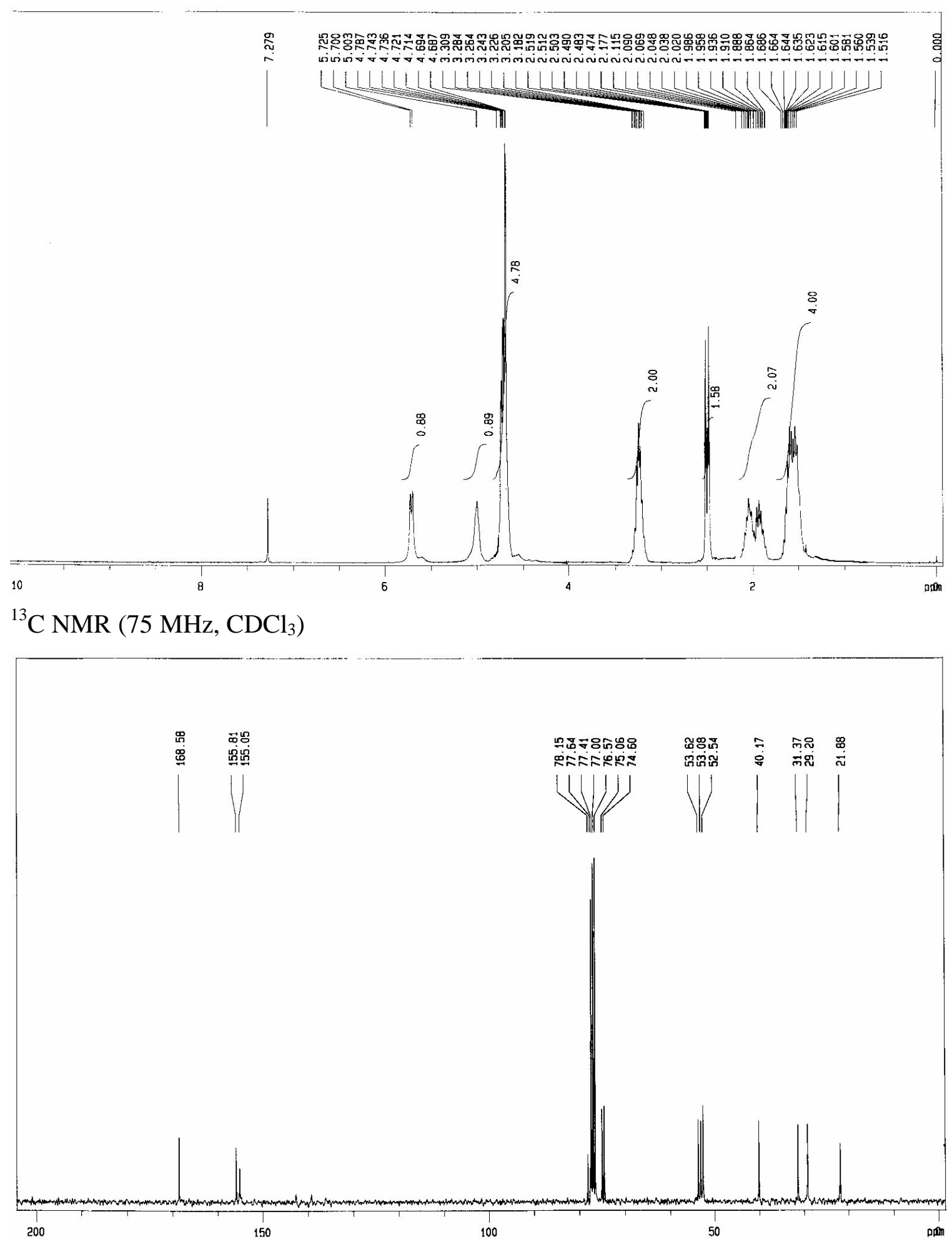


\section{Compound 6p}<smiles>C#CCOC(=O)NC(CCC(=O)Oc1c(F)c(F)c(F)c(F)c1F)C(=O)Oc1c(F)c(F)c(F)c(F)c1F</smiles>

${ }^{19} \mathrm{~F}$ NMR $\left(282 \mathrm{MHz}, \mathrm{CDCl}_{3}\right)$

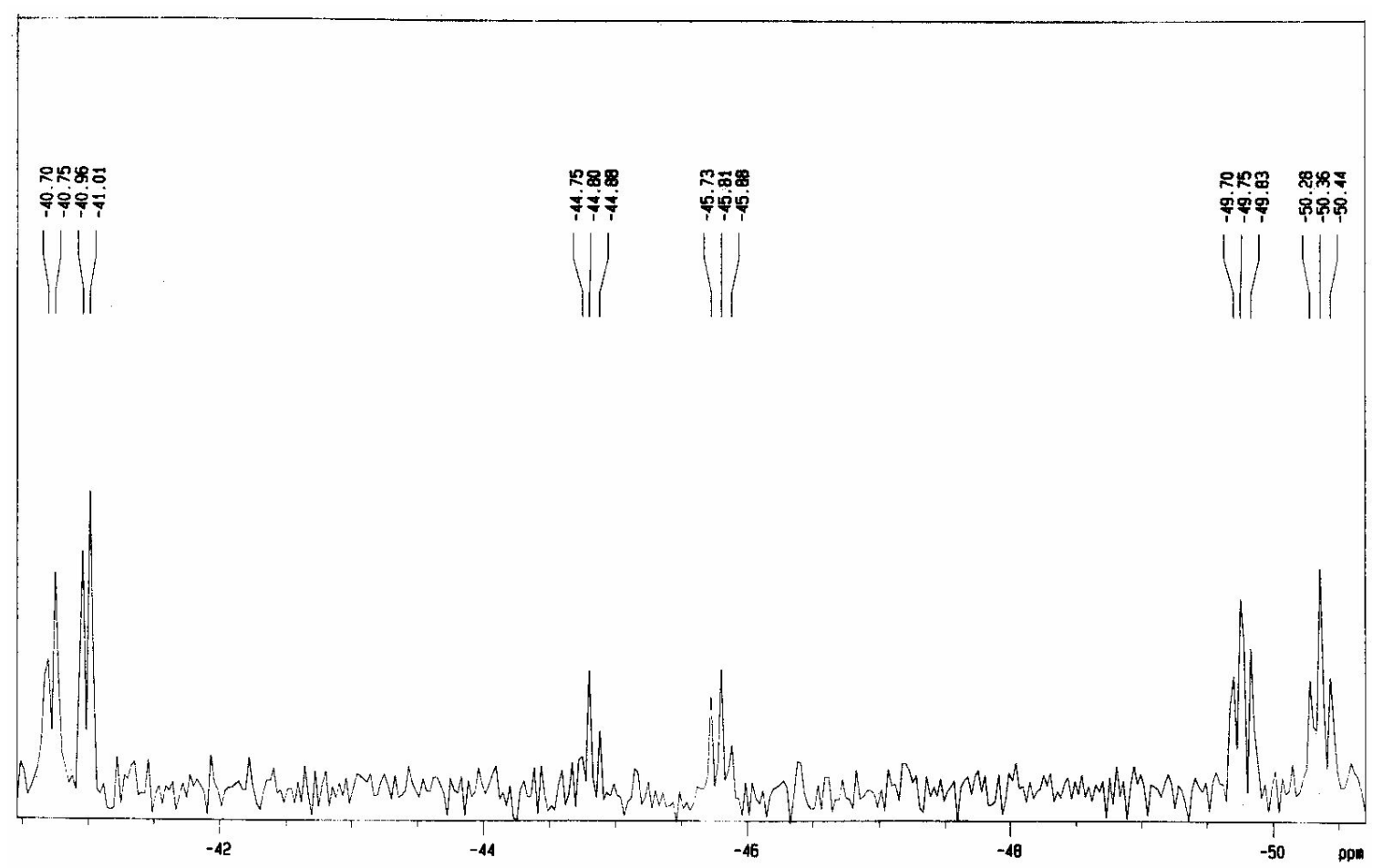




\section{Compound 6p}

${ }^{1} \mathrm{H}$ NMR $\left(300 \mathrm{MHz}, \mathrm{CDCl}_{3}\right.$ )
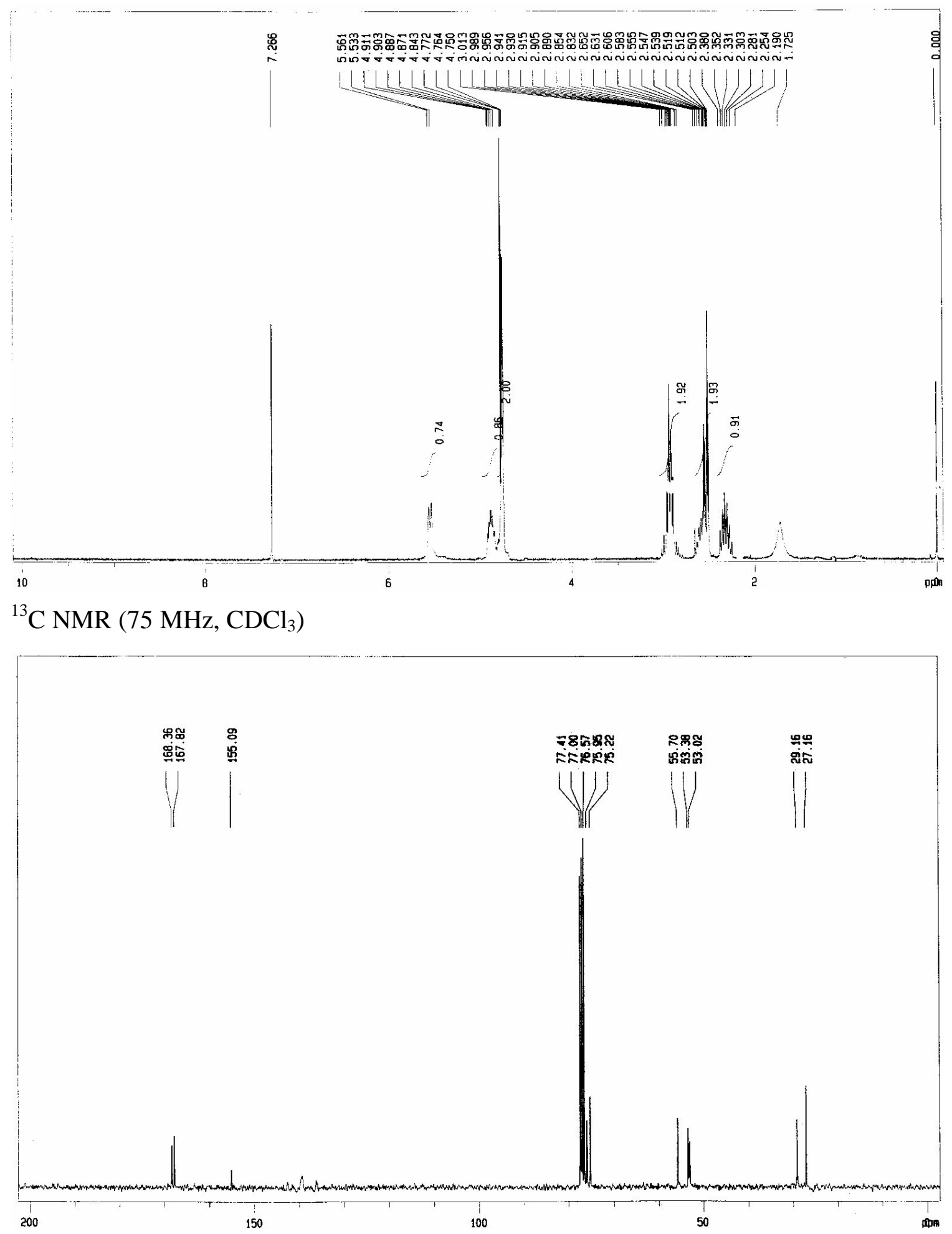


\section{Compound 10}

${ }^{1} \mathrm{H}$ NMR $\left(300 \mathrm{MHz}, \mathrm{CDCl}_{3} / \mathrm{CD}_{3} \mathrm{SOCD}_{3}\right)$
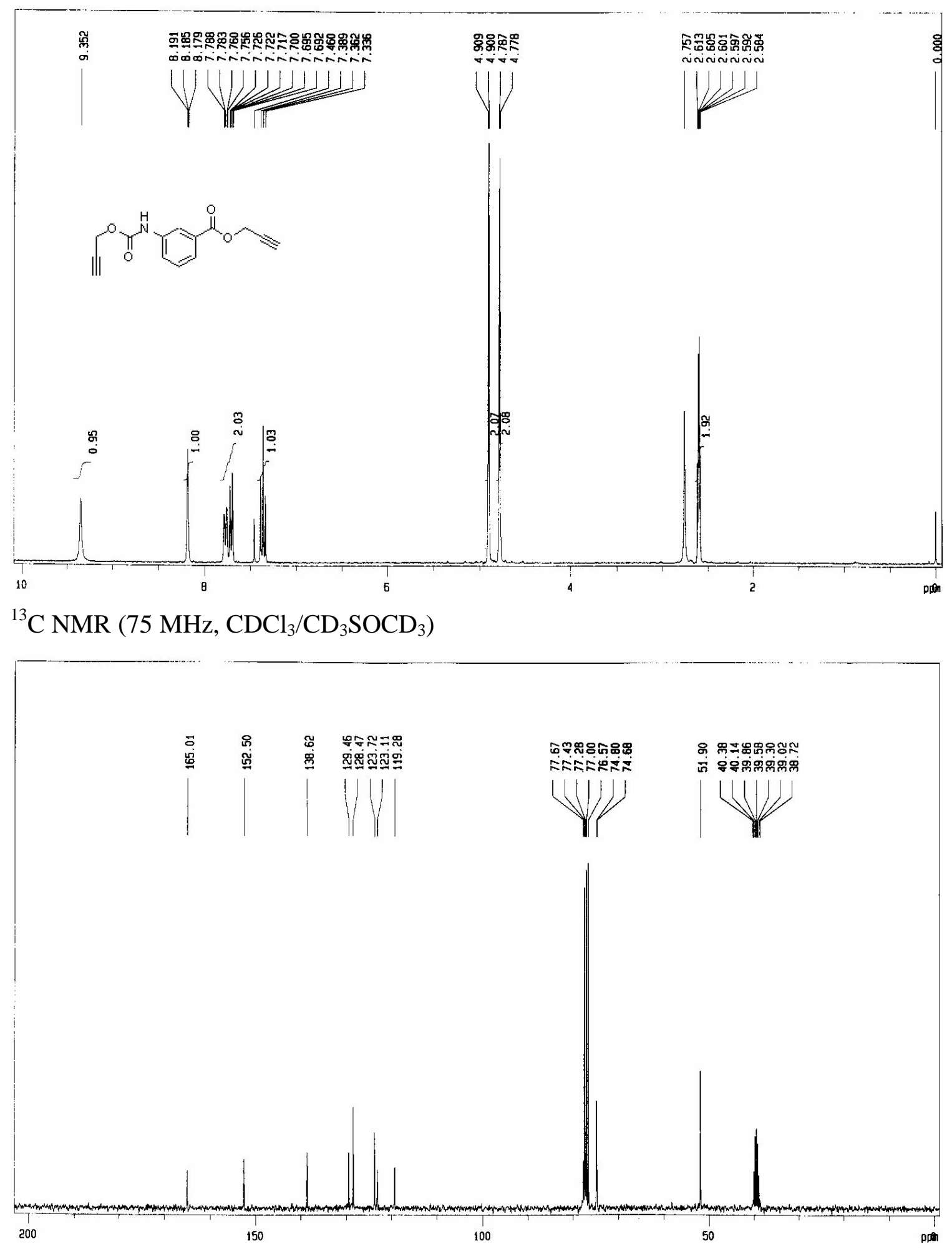